\title{
On the vanishing-viscosity limit in parabolic systems with rate-independent dissipation terms
}

\author{
AleXander Mielke And SERgey ZeliK
}

\begin{abstract}
We consider semilinear and quasilinear parabolic systems with a nonsmooth rate-independent and a viscous dissipation term in the limit of very slow loading rates, or equivalently with fixed loading and vanishing viscosity $\varepsilon>0$. Because for nonconvex energies the solutions will develop jumps, we consider the vanishing-viscosity limit for the graphs of the solutions in the extended state space in arclength parametrization. Here the choice of the viscosity norm for parametrization is crucial to keep the subdifferential structure of the problem. A crucial point in the analysis are new a priori estimates that are rate independent and that allow us to show that the total length of the graph remains bounded in the vanishing-viscosity limit. To derive these estimates we combine parabolic regularity estimates with ideas from rate-independent systems.
\end{abstract}

Mathematics Subject Classification (2010): 35K55 (primary); 34E15 (secondary).

\section{Introduction}

In this paper we are interested in special solution classes for rate-independent systems that arise as vanishing-viscosity limits. In abstract form, we consider doubly nonlinear problems of the following type:

$$
\varepsilon \dot{u}+\partial \tilde{\Psi}(\dot{u})+\mathcal{B} u+\Phi_{u}^{\prime}(t, u) \ni 0, \quad u(0)=u_{0},
$$

where $\varepsilon$ is a small positive parameter. Here $u$ is considered to lie in a Hilbert space $H$ and $\mathcal{B}$ is a positive definite self-adjoint operator generating the scale $H_{\alpha}=$ $D\left(\mathcal{B}^{\alpha / 2}\right)$ of Hilbert spaces. The dissipation potential $\widetilde{\Psi}: H \rightarrow[0, \infty)$ is assumed to be positively homogeneous of degree 1 , such that (1.1) is rate-independent for $\varepsilon=0$ and $\varepsilon>0$ plays the role of a small regularizing viscosity.

For $\varepsilon>0$ the existence of solutions $u_{\varepsilon} \in H^{1}([0, T], H)$ is rather standard using suitable assumptions on the nonlinear potential $\Phi$, see e.g. [6,25]. Under

A.M. was partially supported by DFG under Mi 459/5-2 (Research Unit 797 MicroPlast).

Received April 12, 2010; accepted in revised form February 20, 2012. 
weak hypotheses it is possible to show that these solutions satisfy the basic energy equality

$$
\mathcal{E}\left(t, u_{\varepsilon}(t)\right)+\int_{0}^{t} \widetilde{\Psi}\left(\dot{u}_{\varepsilon}(r)\right)+\varepsilon\left\|\dot{u}_{\varepsilon}(r)\right\|^{2} \mathrm{~d} r=\mathcal{E}\left(0, u_{0}\right)+\int_{0}^{t} \Phi_{r}^{\prime}(r, u(r)) \mathrm{d} r,
$$

where $\mathcal{E}(t, u)=\frac{1}{2}(\mathcal{B} u, u)+\Phi(t, u)$ is the total energy. See also [11, 12,23,26] and references therein for investigations of the long-time behavior of such problems using the attractors theory.

As in $[10,19,20]$ we are interested in the limiting behavior of the solutions $u_{\varepsilon}$ for $\varepsilon \rightarrow 0$. The problem is that in this vanishing-viscosity limit the solutions may develop jumps, and we cannot guarantee that the limit functions or any other solutions is absolutely continuous. Thus, it is not sufficient to impose (1.1) a.e. in $[0, T]$, since we need additional information in jump points.

In $[15,17,22]$ there was developed a fairly general existence theory for socalled energetic solutions to rate-independent systems $(H, \mathcal{E}, \widetilde{\Psi})$ that allows for solutions having jumps. However, these solutions have to satisfy the energy balance (1.2) with $\varepsilon=0$, where $\int_{0}^{t} \widetilde{\Psi}(\dot{u}(r)) \mathrm{d} r$ has to be replaced by the total variation $\int_{0}^{t} \widetilde{\Psi}(\mathrm{d} u)$. It turns out that these solutions are in general not obtained as vanishingviscosity limits, see the discussion in $[13,16,19]$.

Here we follow the ideas in [10,20] by taking the limit $\varepsilon \rightarrow 0$ not for the functions $u_{\varepsilon}:[0, T] \rightarrow H$ but for the trajectories $\left\{\left(t, u_{\varepsilon}(t)\right): t \in[0, T]\right\} \subset$ $[0, T] \times H$. For this we introduce an arc-length parametrization via

$$
s_{\varepsilon}(t)=t+\int_{0}^{t}\left\|\dot{u}_{\varepsilon}(r)\right\| \mathrm{d} r .
$$

Using the inverse $t_{\varepsilon}$ of $s_{\varepsilon}$ we can define $\tilde{u}_{\varepsilon}(s)=u_{\varepsilon}\left(t_{\varepsilon}(s)\right)$ and find the equivalent system

$$
\left\{\begin{array}{l}
\partial \widetilde{\Psi}\left(\tilde{u}^{\prime}(s)\right)+\frac{\varepsilon}{1-\left\|\tilde{u}^{\prime}(s)\right\|} \tilde{u}^{\prime}(s)+\mathcal{B} \tilde{u}(s)+\Phi_{u}^{\prime}(t(s), \tilde{u}(s)) \ni 0 \\
t^{\prime}(s)+\left\|\tilde{u}^{\prime}(s)\right\|=1 \\
u(0)=u_{0}, \quad t(0)=0
\end{array}\right.
$$

where $^{\prime}=\frac{d}{d s}$. The general aim of this paper is to show that a limit $(t(s), \tilde{u}(s))$ of solutions $\left(t_{\varepsilon}(s), \tilde{u}_{\varepsilon}(s)\right)$ of (1.3) satisfies the corresponding limit problem

$$
\left\{\begin{array}{l}
\partial \tilde{\Psi}\left(\tilde{u}^{\prime}(s)\right)+\partial \mathcal{C}_{0}\left(\tilde{u}^{\prime}(s)\right)+\mathcal{B} \tilde{u}(s)+\Phi_{u}^{\prime}(t(s), \tilde{u}(s)) \ni 0 \\
t^{\prime}(s)+\left\|\tilde{u}^{\prime}(s)\right\|=R(s)>0 \\
u(0)=u_{0}, \quad t(0)=0
\end{array}\right.
$$

where $\mathcal{C}_{0}(v)=0$ for $\|v\| \leq 1$ and $\infty$ otherwise. This will in fact be established in various cases, but we cannot guarantee that the parametrization function $R$ in (1.4) 
is equal to 1 . However, we will show certain lower bounds for $R$, see Sections 2.4, 2.5, and 4.3.

The analysis relies heavily on parabolic regularity theory, which implies that so-called weak energetic solutions $u_{\varepsilon} \in L^{\infty}\left([0, T], H_{1}\right) \cap H^{1}([0, T], H)$ of (1.1) are in fact strong solutions satisfying

$$
u_{\varepsilon} \in L^{\infty}\left([t, T], H_{2}\right) \cap H^{1}\left([t, T], H_{1}\right) \cap H^{2}([t, T], H) \text { for all } t \in(0, T) .
$$

However, the norms are not bounded independently of $\varepsilon$. To find suitable uniform estimates we derive the higher-order energy equality

$$
\frac{\varepsilon}{2} \frac{\mathrm{d}}{\mathrm{d} t}\left\|\dot{u}_{\varepsilon}\right\|^{2}+\left(\mathcal{B} \dot{u}_{\varepsilon}, \dot{u}_{\varepsilon}\right)+\left(f_{u}^{\prime}(t, u) \dot{u}, \dot{u}\right)+\left(f_{t}^{\prime}(t, u), \dot{u}\right)=0 .
$$

It is interesting to note that this balance holds independently of the specific choice of the rate-independent dissipation potential $\widetilde{\Psi}$. Because of the nonsmoothness of $\widetilde{\Psi}$ the derivation of this energy balance needs special care. From this we find the a priori estimate

$$
\|u(t)\|_{2}^{2}+\varepsilon^{2}\|\dot{u}(t)\|^{2}+\varepsilon \int_{t}^{t+1}\|\dot{u}(r)\|_{1}^{2} \mathrm{~d} r \leq Q\left(\|u(0)\|_{2}\right) \mathrm{e}^{-\alpha t / \varepsilon}+C_{*},
$$

where $C_{*}, \alpha$ and the monotone function are independent of $\varepsilon, u$, and $t \geq 0$.

To do the vanishing-viscosity limit $\varepsilon \rightarrow 0$, it is necessary to control the integral $\int_{0}^{T}\left\|\dot{u}_{\varepsilon}(t)\right\| \mathrm{d} t$. Again using (1.5) it is possible to derive the estimate

$$
\int_{t_{1}}^{t_{2}}\left\|\dot{u}_{\varepsilon}(t)\right\|_{1} \mathrm{~d} t \leq C\left(1+t_{2}-t_{1}\right)
$$

where the constant $C$ depends on $\left\|u_{0}\right\|_{2}$, but is independent of $\varepsilon, t_{1}$ and $t_{2}$. For this we use some special weighted energy estimates in Section 2.4 or a specially adapted version of Gronwall's estimate in Section 3.4. Both arguments employ an interpolation result that forms a basic assumption in our theory, namely

$$
\exists \theta \in(0,1) \exists C>0:\|u\| \leq C \widetilde{\Psi}(u)^{\theta}\|u\|_{1}^{1-\theta} \text { for all } u \in H_{1} .
$$

This estimate says that the rate-independent dissipation is not too small, such that the trivial a priori estimate for $\int_{0}^{T} \widetilde{\Psi}\left(\dot{u}_{\varepsilon}(t)\right) \mathrm{d} t$ obtained from (1.2) can be employed.

The paper is organized as follows. In Section 2 we treat a concrete situation, where $H=L^{2}(\Omega)^{m}, \mathcal{B}$ is a second-order elliptic operator, and $\widetilde{\Psi}$ and $\Phi$ are classical integral functionals. There we are able to make quite general assumptions and use classical arguments from parabolic regularity. In Section 3 we give the details of the abstract situation as discussed here. Finally, Section 4 treat the more difficult situation where $\mathcal{B}$ is a quasilinear monotone, elliptic operator. In Section 5 we give an application of the theory to a material model involving a coupling between elastic and magnetic behavior, the latter displaying rate-independent hysteresis. 
The parallel developments in the three Sections 2, 3, and 4 hopefully illustrate the different facets of the general approach. While the abstract Section 3 is studied by analogy to the previous section, the other two sections are selfcontained up the following two points. On the one hand, the proof of the semilinear Theorem 2.17 can be obtained by following exactly the steps of the proof of Theorem 4.16. On the other hand, the proof of Proposition 4.11 follows along the lines of the proof of Proposition 2.11.

The method of vanishing viscosity is well-known in many areas of mathematics. For rate-independent systems this approach was studied in several applications, like in crack propagation $[13,14,27]$, for fracture [5], for plasticity [7, 8]. However, only recently the limiting solutions are characterized sufficiently well along the jumps. In $[19,20]$ a notion of BV solutions was introduced that avoids the arclength parametrization employed here. Moreover, from the results there it seems reasonable to expect that limit points of the unscaled solution $u_{\varepsilon}:[0, T] \rightarrow H$ are BV solutions. Some first results are obtained in $[19,20]$ in the finite-dimensional setting, while the infinite-dimensional case will be studied in [21]; see also [16] for some preliminary results.

In Section 3.6 it is shown that the solutions constructed here are BV solutions in the sense of $[20,21]$. However, our parametrization with the viscous norm $\|\cdot\|$ of $H$ needs stronger assumptions on the energy functional compared to the more general notion of BV solutions. In return, we obtain a better characterization of of the limiting solutions; in particular, we maintain the subdifferential structure (1.4) matching the original subdifferential form (1.1) of the problem.

A different reparametrization is chosen in $[4,8]$ for nonconvex elastoplastic models and in [19-21] for general systems. There the dissipated energy is used to define arclength, namely

$$
s_{\varepsilon}(t)=t+\int_{0}^{t}\left\|\widetilde{\Psi}\left(\dot{u}_{\varepsilon}(\tau)\right)\right\|+\operatorname{dist}_{\|\cdot\|_{*}}\left(-\mathcal{B} u_{\varepsilon}(\tau)-\Phi_{u}^{\prime}\left(\tau, u_{\varepsilon}(\tau)\right), \mathcal{K}^{*}\right) \mathrm{d} \tau,
$$

where $\mathcal{K}^{*}=\partial \tilde{\Psi}(0) \subset H^{*}$. The last term involving the distance from $\mathcal{K}^{*}$ was introduced in $\left[19\right.$, Eqn. (33)] for characterizing BV solutions $\left(c f\right.$. the term $v(\xi-1)^{+}$ in $M_{0}(\alpha, v, \xi)$ there). It also appears in the vanishing-viscosity potential $\mathfrak{p}$ in [16, Def. 4.20] and in the term $\mathbb{J}$ in Section 3.6 on BV solutions, see (3.25e).

ACKNOWLEDGEMENTS. The authors are grateful to Riccarda Rossi and Giuseppe Savaré for helpful discussions. They also thank two unknown referees for spotting several inconsistencies.

\section{The semi-linear case}

This chapter is devoted to the study of the semi-linear case of equations (1.1) where the leading part $\mathcal{B} u$ is a linear second order differential operator. The rigorous formulation of the problem, assumptions on $\Psi, \mathcal{B}$ and $F$ and some preliminary 
facts are collected in Section 2.1. The uniform with respect to $\varepsilon \rightarrow 0$ estimates for (1.1) together with the existence and uniqueness of a solution are proved in Section 2.2. In particular, the crucial higher energy identity (1.5) is verified there. The passage to the rate-independent limit $\varepsilon \rightarrow 0$ in equations (1.3) is justified in Section 2.4 and the strict positivity of the scaling factor $R(s)$ in the limit problem (1.4) is verified in Section 2.5.

The example of a problem in the form (1.1) for which $R(s)$ is strictly positive is constructed in Section 2.6. We have to mention that this example is not entirely satisfactory since the nonlinearity in it has the additional small non-gradient part, however, all of the above theory works for that case as well.

Finally, the abstract semilinear case is briefly discussed in Section 3. In particular, the alternative approach to establishing the higher energy estimates which does not require the approximation of the non-smooth functional $\Psi$ by smooth ones is indicated here.

\subsection{Assumptions and preliminaries}

In this section we start to study the semi-linear version of our main equation which has the following form:

$$
\left\{\begin{array}{l}
\varepsilon \partial_{t} u+\partial \Psi\left(\partial_{t} u\right)+\mathcal{B} u+f(t, u) \ni 0 \\
\left.u\right|_{\partial \Omega}=0, \quad u(0, \cdot)=u_{0}
\end{array}\right.
$$

where $u(t, x)=\left(u^{1}(t, x), \cdots, u^{m}(t, x)\right)$ is an unknown vector-valued function, $\Omega \Subset \mathbb{R}^{n}$ is a bounded smooth domain in $\mathbb{R}^{n}$ and $\varepsilon>0$ is a small positive parameter.

We assume that $\mathcal{B}$ is a linear second order self-adjoint elliptic operator in $\left[L^{2}(\Omega)\right]^{m}$ satisfying

$$
\mathcal{B}^{*}=\mathcal{B}, \quad(\mathcal{B} u, u)_{L^{2}} \geq \kappa\|u\|_{H^{1}}^{2} \text { for all } u \in H_{0}^{1}(\Omega)=W_{0}^{1,2}(\Omega)
$$

for some positive constant $\kappa$. Here and below, $W^{l, p}(\Omega)$ is a Sobolev space of functions whose distributional derivatives up to order $l$ belong to $L^{p}(\Omega), W_{0}^{l, p}(\Omega)$ is the closure of $C_{0}^{\infty}(\Omega)$ in the space $W^{l, p}(\Omega)$ and $(u, v)_{L^{2}}$ is a usual inner product in $L^{2}(\Omega)$ (we will also use this notation for the duality between the Sobolev spaces $W_{0}^{l, p}(\Omega)$ and $\left.W^{-l, q}(\Omega)=\left[W_{0}^{l, p}(\Omega)\right]^{*}, \frac{1}{p}+\frac{1}{q}=1\right)$. Moreover, the spaces $H^{s}(\Omega)$ denote the Hilbert spaces $W^{s, 2}(\Omega)$. We will further use $\|\cdot\|_{L^{p}}$ and $\|\cdot\|_{H^{s}}$ to indicate the norms in $L^{p}(\Omega)$ and $H^{s}(\Omega)$, respectively.

The dissipation potential $\Psi: \mathbb{R}^{m} \rightarrow[0, \infty)$ is assumed to be coercive, convex, and positively homogeneous of degree 1 , namely

$$
\begin{aligned}
& \exists \beta_{1}, \beta_{2}>0: \beta_{1}|v| \leq \Psi(v) \leq \beta_{2}|v| \text { for all } v \in \mathbb{R}^{m}, \\
& \Psi(\alpha v)=\alpha \Psi(v) \text { for all } \alpha \geq 0 \text { and } v \in \mathbb{R}^{m}, \\
& \Psi(v+w) \leq \Psi(v)+\Psi(w) \text { for all } v, w \in \mathbb{R}^{m} .
\end{aligned}
$$


By $\partial \Psi(v)$ we denote the multivalued subdifferential of $\Psi$, i.e.,

$$
\partial \Psi(v)=\left\{\eta \in \mathbb{R}^{m} \mid \Psi(w+v) \geq \Psi(v)+\eta . w \text { for all } w \in \mathbb{R}^{m}\right\} .
$$

Here and below $u . v$ means the inner product in $\mathbb{R}^{m}$.

Finally the nonlinearity $f: \mathbb{R} \times \mathbb{R}^{m} \rightarrow \mathbb{R}^{m}$ is supposed to be potential, i.e.,

$$
f(t, u):=F_{u}^{\prime}(t, u) \text { for some } F \in C^{2}\left(\mathbb{R} \times \mathbb{R}^{m}, \mathbb{R}\right),
$$

and to satisfy the growth restrictions

$$
|u| \cdot\left|f_{u}^{\prime}(t, u)\right|+\left|f_{t}^{\prime}(t, u)\right| \leq C\left(1+|u|^{r+1}\right),
$$

where $0<r<r_{\max }:=\frac{4}{n-2}$ for $n \geq 3$ and $r_{\max }=\infty$ otherwise, and the dissipativity assumption

$$
f(t, u) \cdot u \geq-K+\kappa_{1}|u|^{r+2} .
$$

Assumption of (2.6) is a standard (parabolic) dissipativity condition, see $e . g$. [2]. In particular, the growth restrictions (2.5) and the dissipativity assumption (2.6) imply

$$
\begin{aligned}
& f(t, u) \cdot u \geq-C, \\
& F(t, u) \leq \kappa_{3} f(t, u) \cdot u+C\left(1+|u|^{2}\right), \\
& F_{t}^{\prime}(t, u) \leq \kappa_{3} f(t, u) \cdot u+C\left(1+|u|^{2}\right), \quad \kappa_{3}>0 .
\end{aligned}
$$

In order to simplify the passage to the abstract semilinear case, we collect all necessary analytic properties of the nonlinearity $f$ in the next lemma. It quantifies the fact that $f(t, \cdot)$ is subordinated to the main elliptic part $\mathcal{B} u$.

Lemma 2.1. Let the function $f$ satisfy growth restrictions (2.5). Then, there exists $\gamma>0$ and a monotone function $Q:[0, \infty) \rightarrow[0, \infty)$ such that the following holds:

(1) The operators $u \mapsto f(t, u)$ and $u \mapsto f_{t}^{\prime}(t, u)$ are continuous operators from $H^{1-\gamma}$ to $H^{-1+\gamma}$ satisfying the estimate

$$
\|f(t, u)\|_{H^{-1+\gamma}}+\left\|f_{t}^{\prime}(t, u)\right\|_{H^{-1+\gamma}} \leq Q\left(\|u\|_{H^{1-\gamma}}\right) ;
$$

(2) The functionals $u \mapsto \Phi(t, u):=(F(t, u), 1)_{L^{2}}$ and $u \mapsto \Phi_{t}^{\prime}(t, u)=\left(F_{t}^{\prime}(t, u), 1\right)_{L^{2}}$ are continuous on $H^{1-\gamma}$ and satisfy the estimate

$$
|\Phi(t, u)|+\left|\Phi_{t}^{\prime}(t, u)\right| \leq Q\left(\|u\|_{H^{1-\gamma}}\right)
$$

(3) The functional $(u, v) \mapsto\left(f_{u}^{\prime}(t, u) v, v\right)_{L^{2}}$ is continuous on $H^{1-\gamma} \times H^{1-\gamma}$ with

$$
\left|\left(f_{u}^{\prime}(t, u) v, v\right)_{L^{2}}\right| \leq Q\left(\|u\|_{H^{1-\gamma}}\right)\|v\|_{H^{1-\gamma}}^{2}
$$


(4) Let, in addition, $n \leq 6$, then for $u \in H^{2}(\Omega)$ the following estimates hold:

$$
\begin{aligned}
& \|f(t, u)\|_{L^{2}} \leq Q\left(\|u\|_{H^{1}}\right)\|u\|_{H^{2}}^{1-\gamma}, \\
& \left\|f_{u}^{\prime}(t, u) v\right\|_{L^{2}} \leq Q\left(\|u\|_{H^{1}}\right)\left(1+\|u\|_{H^{2}}\right)\|v\|_{H^{1}}, \\
& \left\|f_{t}^{\prime}(t, u)\right\|_{L^{2}} \leq Q\left(\|u\|_{H^{2}}\right) .
\end{aligned}
$$

Proof. The assertions of this lemma are more or less standard, thus we leave the rigorous proof to the reader, more details can be found in the proof of Lemma 4.1. We just indicate how the additional restriction $n \leq 6$ appears for establishing (2.11b). Moreover, without loss of generality, we consider the case $n \geq 3$ only (the case $n \leq 2$ is trivial since $H^{1} \subset L^{p}$ for every $p$ ) and assume even the weaker condition on $r: r=\frac{4}{n-2}$ (which is sufficient for proving that estimate).

Indeed, due to the growth restriction on $f_{u}^{\prime}$ and Hölder inequality with exponents $n / 2$ and $n /(n-2)$, we have

$$
\left\|f_{u}^{\prime}(t, u) v\right\|_{L^{2}}^{2} \leq C\left(1+\|u\|_{L^{4 n /(n-2)}}^{8 /(n-2)}\|v\|_{L^{2 n /(n-2)}}^{2} .\right.
$$

Using now the Sobolev's embedding theorem $H^{s} \subset L^{p(s)}$ where $1 / p(s)=1 / 2-$ $s / n$ (actually, we use it twice with $s=1, p=2 n /(n-2)$ and $s=1 / 2+n / 4$ and $p=4 n /(n-2))$, we arrive at

$$
\left\|f_{u}^{\prime}(t, u) v\right\|_{L^{2}}^{2} \leq C\left(1+\|u\|_{H^{1 / 2+n / 4}}^{8 /(n-2)}\right)\|v\|_{H^{1}}^{2} .
$$

We see that the condition $n \leq 6$ guarantees that the exponent $1 / 2+n / 4$ will not exceed 2 and the interpolation

$$
\|u\|_{H^{1 / 2+n / 4}} \leq C\|u\|_{H^{1}}^{(6-n) / 4}\|u\|_{H^{2}}^{(n-2) / 4}
$$

gives the desired estimate (2.11b). Lemma 2.1 is proved.

Remark 2.2. It worth to mention that the restriction $n \leq 6$ is necessary only if the exponent $r$ is close to $r_{\max }$; it can be relaxed otherwise. In particular, in the case $r \leq \frac{2}{n-2}$, estimate (2.11b) holds for all $n$.

In order to study the dependence of solutions of problem (2.1) on $\varepsilon$, it is however more convenient to scale time $\tau=\varepsilon t$ and rewrite the problem in the equivalent form with respect to the function $\tilde{u}(\tau):=u(\varepsilon \tau)$. In order to simplify the notations, we will write below $u(\tau)$ instead of $\tilde{u}(\tau)$. We essentially use that $\Psi$ is positively homogeneous of order 1 and, consequently, $\partial \Psi$ is positively homogeneous of order 0 . Equation (2.1) reads

$$
\left\{\begin{array}{l}
\partial_{\tau} u+\partial \Psi\left(\partial_{\tau} u\right)+\mathcal{B} u+f_{\varepsilon}(\tau, u) \ni 0, \\
\left.u\right|_{\partial \Omega}=0,\left.\quad u\right|_{\tau=0}=u_{0},
\end{array}\right.
$$

where, by $f_{\varepsilon}(\tau, u):=f(\varepsilon \tau, u)$.

We are now ready to recall the definition of weak energy solutions for problem (2.12). 
Definition 2.3. Let the functions $\Psi$ and $f$ and the operator $\mathcal{B}$ satisfy the above assumptions. A function $u:[0, R] \rightarrow L^{2}(\Omega)$ is an energy solution of problem (2.12), if

$$
u \in L^{\infty}\left([0, R], H_{0}^{1}(\Omega)\right) \text { and } \partial_{\tau} u \in L^{2}\left([0, R], L^{2}(\Omega)\right)
$$

and if it satisfies equation (2.12) in the sense of distributions.

The next lemma gives some immediate additional regularity of energy solutions which is necessary to establish the uniqueness.

Lemma 2.4. Let the above assumptions hold. Then, every energy solution $u$ of problem (2.12) satisfies

$$
\text { (a) } u \in L^{2}\left([0, R], H^{2}(\Omega)\right), \quad \text { (b) } f_{\varepsilon}(\cdot, u) \in L^{2}\left([0, R], L^{2}(\Omega)\right)
$$

and, in particular, equation (2.12) can be understood as an equality in $L^{2}([0, R]$, $\left.L^{2}(\Omega)\right)$.

Proof. Indeed, since the term $\partial \Psi\left(\partial_{\tau} u\right)$ is uniformly bounded in $L^{\infty}$, condition (2.13) gives

$$
\Theta_{u}:=-\mathcal{B} u-f_{\varepsilon}(\cdot, u) \in L^{2}\left([0, R], L^{2}(\Omega)\right) .
$$

Since $\mathcal{B}$ is elliptic and $\Omega$ is smooth, we have the $H^{2}$-regularity result for $\mathcal{B}$, namely

$$
\|u\|_{H^{2}(\Omega)} \leq C\|\mathcal{B} u\|_{L^{2}},
$$

see $e . g .$, [28]. The presence of the additional subordinated term $f_{\varepsilon}(\tau, u)$ does not destroy the maximal regularity estimate (2.16). Indeed, due to (2.11a) we obtain the interpolation estimate $\left\|f_{\varepsilon}(\tau, u)\right\|_{L^{2}} \leq Q_{\beta}\left(\|u\|_{H^{1}}\right)+\beta\|u\|_{H^{2}(\Omega)}$, where $\beta>0$ is arbitrary and $Q_{\beta}$ is an appropriate monotone function. Applying the elliptic regularity estimate (2.16) together with the last estimate to equation (2.15) and taking $\beta>0$ being small enough, we have

$$
\|u(\tau)\|_{H^{2}(\Omega)} \leq C\left\|\Theta_{u}(\tau)\right\|_{L^{2}}+Q\left(\|u(\tau)\|_{H^{1}}\right) .
$$

Employing (2.13) the assertion (2.14)(a) follows. It only remains to note that (2.14)(b) is an immediate consequence of (2.11a) and (2.14)(a).

Corollary 2.5. Every weak energy solution of problem (2.12) satisfies the so-called energy equality, i.e., for all $0 \leq \tau_{1}<\tau_{2} \leq R$ we have

$$
\begin{aligned}
& \left.\frac{1}{2}\left(\mathcal{B} u\left(\tau_{2}\right), u\left(\tau_{2}\right)\right)_{L^{2}}-\frac{1}{2}\left(\mathcal{B} u\left(\tau_{1}\right)\right), u\left(\tau_{1}\right)\right)_{L^{2}} \\
& \quad+\int_{\tau_{1}}^{\tau_{2}}\left\|\partial_{\tau} u(\tau)\right\|_{L^{2}}^{2}+\left(\Psi\left(\partial_{\tau} u(\tau)\right), 1\right)_{L^{2}} \mathrm{~d} \tau \\
& =-\int_{\tau_{1}}^{\tau_{2}}\left(f_{\varepsilon}(\tau, u(\tau)), \partial_{\tau} u(\tau)\right)_{L^{2}} \mathrm{~d} \tau .
\end{aligned}
$$


Indeed, multiplying equation (2.12) by $\partial_{\tau} u$ (which is possible to do since all of the terms in (2.12) belongs to $L^{2}([0, R] \times \Omega)$ and $\partial_{\tau} u$ also belongs to that space), integrating over $(\tau, x) \in[0, R] \times \Omega$ and using the standard fact that

$$
\partial \Psi(u) . u=\Psi(u),
$$

we end up with identity (2.18). Moreover, using the potential $F$ from (2.4) we may introduce the stored energy functional $\mathcal{E}:[0, T] \times H^{2}(\Omega) \rightarrow \mathbb{R}$ via $\mathcal{E}(t, u)=$ $\frac{1}{2}(\mathcal{B} u, u)+(F(t, u), 1)$. Вy $\widetilde{\Psi}: v \mapsto(\Psi(v), 1)=\int_{\Omega} \Psi(v(x)) \mathrm{d} x$ we denote the dissipation potential associated with $\Psi$. With this the energy equation (2.18) can be rewritten as

$\mathcal{E}\left(\tau_{2}, u\left(\tau_{2}\right)\right)+\int_{\tau_{1}}^{\tau_{2}}\left(\tilde{\Psi}\left(\partial_{\tau} u\right)+\left\|\partial_{\tau} u\right\|_{L^{2}}^{2}\right) \mathrm{d} \tau=\mathcal{E}\left(\tau_{1}, u\left(\tau_{1}\right)\right)+\int_{\tau_{1}}^{\tau_{2}} \partial_{\tau} \mathcal{E}(\tau, u(\tau)) \mathrm{d} \tau$,

where $\partial_{\tau} \mathcal{E}(\tau, u(\tau)):=\left(f_{\mathcal{E}, \tau}^{\prime}(\tau, u(\tau)), 1\right)$. This is the form used in [15,19].

It is well-known that, under the above assumptions, there exists at least one weak energy solution of problem (2.12) (see e.g., [6]). Moreover, as we will see in the next section, this solution is unique. However, the regularity (2.13) and (2.14) seem insufficient to pass to the rate independent limit $\varepsilon \rightarrow 0$ and we need a stronger notion of solutions for problem (2.12).

Definition 2.6. We say that a function $u=u(\tau, x)$ is a strong solution of (2.12) if, for every $R>0$,

$$
\begin{aligned}
& \text { (a) } u \in L^{\infty}\left([0, R], H^{2}(\Omega)\right) \\
& \text { (b) } \partial_{\tau} u \in L^{\infty}\left([0, R], L^{2}(\Omega)\right) \cap L^{2}\left([0, R], H^{1}(\Omega)\right)
\end{aligned}
$$

and $u$ satisfies equation (2.12) in the sense of distributions.

Let us now briefly explain how to obtain the additional regularity (2.19), this derivation will be justified in the next section. Let us (formally) differentiate equation (2.12) by $\tau$ and denote $v=\partial_{\tau} u$. Then, we have

$$
\partial_{\tau} v+\partial^{2} \Psi(v) \partial_{\tau} v=\mathcal{B} v-f_{\varepsilon, u}^{\prime}(\tau, u) v-f_{\varepsilon, \tau}^{\prime}(\tau, u) .
$$

Furthermore, Euler's identity for one-homogeneous functions gives (again formally)

$$
\partial^{2} \Psi(v) \partial_{\tau} v . v=\partial_{\tau}[\partial \Psi(v) . v]-\partial \Psi(v) . \partial_{\tau} v=\partial_{\tau}[\partial \Psi(v) . v-\Psi(v)]=0 .
$$

Multiplying now equation (2.20) by $v$ and integrating over $x \in \Omega$, we have

$$
\frac{1}{2} \frac{d}{d \tau}\|v(\tau)\|_{L^{2}}^{2}+(\mathcal{B} v(\tau), v(\tau))_{L^{2}}+\left(f_{\varepsilon, u}^{\prime}(\tau, u(\tau)) v(\tau)+f_{\varepsilon, \tau}^{\prime}(\tau, u(\tau)), v(\tau)\right)_{L^{2}}=0
$$

The main observation is that the terms containing $\Psi$ disappears in all the estimates. This is due to the homogeneity of $\Psi$ leading to the identity (2.21). Thus, we obtain 
the same equations as estimates as in the case $\Psi=0$, which would be a smooth partial differential equation.

Integrating this identity by $\tau$ and using estimates (2.9) and (2.10) one can deduce the desired estimate for the function $v$ in $L^{2}\left([0, R], H^{1}(\Omega)\right)$ and $L^{\infty}([0, R]$, $\left.L^{2}(\Omega)\right)$. Finally, the estimate for $u$ in $L^{\infty}\left([0, R], H^{2}(\Omega)\right)$ follows using elliptic regularity estimate applied to equation (2.15).

Of course, the above formal arguments are far from being a rigorous proof, since the possibility to differentiate equation (2.12) by $\tau$ and identity (2.21) are not evident especially due to the presence of the non-smooth term $\Psi$ (we recall that one-homogeneous functional $\Psi(v)$ is even not $C^{1}$ at $v=0$ ) and require an accurate justification. Nevertheless, as we will see below, every weak energy solution is automatically a strong solution of (2.12) if $u_{0} \in H^{2}(\Omega)$.

\subsection{Classical a priori estimates, existence and uniqueness}

The aim of this section is to study energy solutions and strong solutions for problem (2.12) and to verify a number of uniform estimates with respect to $\varepsilon$. They are necessary for passing to the rate independent limit $\varepsilon \rightarrow 0$. We start with the uniqueness of energy solutions.

Proposition 2.7. Let the functions $\Psi$ and $f$ and the operator $\mathcal{B}$ satisfy the assumptions of the previous section (in particular, assume (2.11b)) and let $u_{0} \in H_{0}^{1}(\Omega)$. Then, the energy solution of problem (2.12) is unique and, for every two energy solutions $u_{1}$ and $u_{2}$ (with different initial data belonging to $H_{0}^{1}(\Omega)$ ) the following Lipschitz continuity holds:

$$
\left\|u_{1}(\tau)-u_{2}(\tau)\right\|_{H^{1}} \leq C \mathrm{e}^{K \tau}\left\|u_{1}(0)-u_{2}(0)\right\|_{H^{1}}
$$

where the constants $C$ and $K$ depends only on the energy norms of $u_{1}$ and $u_{2}$.

Proof. For two energy solutions $u_{1}$ and $u_{2}$ of (2.12) let $w(\tau):=u_{1}(\tau)-u_{2}(\tau)$. Then,

$$
\left[\partial \Psi\left(\partial_{\tau} u_{1}\right)-\partial \Psi\left(\partial_{\tau} u_{2}\right)\right]+\partial_{\tau} w=-\mathcal{B} w-\left[f_{\varepsilon}\left(u_{1}\right)-f_{\varepsilon}\left(u_{2}\right)\right] .
$$

Multiplying this equation by $\partial_{\tau} w(\tau)$, integrating over $\Omega$ and using the monotonicity of $\partial \Psi\left(\right.$ i.e., $\left.\left(\partial \Psi\left(\partial_{\tau} u_{1}\right)-\partial \Psi\left(\partial_{\tau} u_{2}\right), \partial_{\tau} u_{1}-\partial_{\tau} u_{2}\right)_{L^{2}} \geq 0\right)$, we obtain

$$
\frac{d}{d \tau}(\mathcal{B} w(\tau), w(\tau))_{L^{2}} \leq\left\|f_{\varepsilon}\left(\tau, u_{1}(\tau)\right)-f_{\varepsilon}\left(\tau, u_{2}(\tau)\right)\right\|_{L^{2}}^{2} .
$$

Thus, we only need to estimate the $L^{2}$ norm in the right-hand side. To this end we note that, due to estimate (2.11b),

$$
\begin{aligned}
& \left\|f_{\varepsilon}\left(u_{1}(\tau)\right)-f_{\varepsilon}\left(u_{2}(\tau)\right)\right\|_{L^{2}} \\
& \leq \int_{0}^{1}\left\|f_{\varepsilon, u}\left(s u_{1}(\tau)+(1-s) u_{2}(\tau)\right) w\right\|_{L^{2}} \mathrm{~d} s \\
& \leq Q\left(\left\|u_{1}(\tau)\right\|_{H^{1}}+\left\|u_{2}(\tau)\right\|_{H^{1}}\right)\left(1+\left\|u_{1}(\tau)\right\|_{H^{2}}+\left\|u_{2}(\tau)\right\|_{H^{2}}\right)\|w\|_{H^{1}} \\
& :=L(\tau)\|w\|_{H^{1}} .
\end{aligned}
$$


Inserting (2.25) into (2.24) and using (2.2) for operator $\mathcal{B}$, we infer

$$
\frac{d}{d \tau}(\mathcal{B} w(\tau), w(\tau))_{L^{2}} \leq C L^{2}(\tau)(\mathcal{B} w(\tau), w(\tau))_{L^{2}}
$$

Taking now into account (2.13) and (2.14), we see that $\int_{0}^{\tau} L(r)^{2} \mathrm{~d} r \leq C(\tau+1)$, where the constant $C$ depends only on the energy norms of $u_{1}$ and $u_{2}$. The Gronwall estimate applied to (2.26) gives now the required estimate (2.23) and finishes the proof.

Our next task is to construct a strong solution of problem (2.12) and to justify the formal derivation of estimate (2.22) given in a previous subsection. To this end, we approximate the function $\Psi$ by smooth ones, namely $\Psi_{\delta}$ with

$$
\Psi_{\delta}(v):=\int_{\mathbb{R}^{m}} \delta^{-m} \varphi(r / \delta) \Psi(v-r) \mathrm{d} r
$$

where $\varphi$ is a standard smoothing kernel in $\mathbb{R}^{m}$, see Appendix A for the detailed study of the approximating functions $\Psi_{\delta}$. We consider the smoothed version of problem (2.12)

$$
\left\{\begin{array}{l}
\partial_{\tau} u+\Psi_{\delta}^{\prime}\left(\partial_{\tau} u\right)+\mathcal{B} u+f_{\varepsilon}(\tau, u)=0, \\
\left.u\right|_{\partial \Omega}=0, \quad u(0, \cdot)=u_{0}
\end{array}\right.
$$

Our task now is to obtain estimates for the solutions of this auxiliary problem, which are uniform with respect to $\varepsilon$ and $\delta$. The required strong solution $u(\tau)$ of the initial problem (2.12) will then be obtained in the limit $\delta \rightarrow 0$. The next theorem gives the existence of strong solutions for that auxiliary problem.

Theorem 2.8. Let the assumptions of Proposition 2.7 hold and let $u_{0} \in H^{2}(\Omega)$. Then, for every $\delta>0$, there exists a unique strong solution $u=u_{\delta}$ of problem (2.27). This solution belongs to $A C\left(\mathbb{R}_{+}, H_{0}^{1}(\Omega)\right)$, satisfies the energy identity

$$
\begin{aligned}
& \left.\frac{1}{2}\left(\mathcal{B} u\left(\tau_{2}\right), u\left(\tau_{2}\right)\right)_{L^{2}}-\frac{1}{2}\left(\mathcal{B} u\left(\tau_{1}\right)\right), u\left(\tau_{1}\right)\right)_{L^{2}} \\
& \quad+\int_{\tau_{1}}^{\tau_{2}}\left\|\partial_{\tau} u(\tau)\right\|_{L^{2}}^{2}+\left(\Psi_{\delta}^{\prime}\left(\partial_{\tau} u(\tau)\right), \partial_{\tau} u(\tau)\right)_{L^{2}} \mathrm{~d} \tau \\
& =-\int_{\tau_{1}}^{\tau_{2}}\left(f_{\varepsilon}(\tau, u(\tau)), \partial_{\tau} u(\tau)\right)_{L^{2}} \mathrm{~d} \tau
\end{aligned}
$$

for all $0 \leq \tau_{1} \leq \tau_{2}$ and the dissipative estimate

$$
\|u(\tau)\|_{H^{2}(\Omega)}^{2}+\left\|\partial_{\tau} u(\tau)\right\|_{L^{2}}^{2}+\int_{\tau}^{\tau+1}\left\|\partial_{\tau} u(r)\right\|_{H^{1}}^{2} \mathrm{~d} r \leq Q\left(\|u(0)\|_{H^{2}(\Omega)}\right) \mathrm{e}^{-k \tau}+C_{f},
$$


where the positive constants $k$ and $C_{f}$ and the monotone function $Q$ are independent of $\tau, \varepsilon$ and $\delta$. In addition, the solution $u$ satisfies the following integrated version of (2.22):

$$
\begin{aligned}
& \left\|\partial_{\tau} u\left(\tau_{2}\right)\right\|_{L^{2}}^{2}-\left\|\partial_{\tau} u\left(\tau_{1}\right)\right\|_{L^{2}}^{2}+2 \kappa \int_{\tau_{1}}^{\tau_{2}}\left\|\partial_{\tau} u(\tau)\right\|_{H^{1}}^{2} \mathrm{~d} \tau \\
& \left.\quad+2 \int_{\tau_{1}}^{\tau_{2}}\left(f_{\varepsilon, \tau}^{\prime}(\tau, u(\tau))+f_{\varepsilon, u}^{\prime}(\tau, u(\tau)) \partial_{\tau} u(\tau), \partial_{\tau} u(\tau)\right)_{L^{2}}\right] \mathrm{d} \tau \leq C \delta
\end{aligned}
$$

for all $0 \leq \tau_{1} \leq \tau_{2}$, where $\kappa>0$ is the same as in (2.2) and $C$ is independent of $\delta, \tau_{1}, \tau_{2}$ and $u$. Finally, $\partial_{\tau} u \in L^{\infty}\left((\tau, R], H^{1}(\Omega)\right)$ and $\left.\partial_{\tau}^{2} u \in L^{2}((\tau, R] \times \Omega)\right)$ for every $\tau>0$ and the following estimate holds

$$
\left\|\partial_{\tau} u(\tau)\right\|_{H^{1}}^{2}+\int_{\tau}^{\tau+1}\left\|\partial_{\tau}^{2} u(r)\right\|_{L^{2}}^{2} \mathrm{~d} r \leq \frac{\tau+1}{\tau} Q\left(\|u(0)\|_{H^{2}}\right) \mathrm{e}^{-k \tau}+C_{f},
$$

where $Q, C_{f}>0$, and $k$ are independent of $\delta, \tau$ and $u$.

Proof. The energy equality (2.28) can be obtained multiplying equation (2.27) by $\partial_{\tau} u$ exactly as in Corollary 2.5 . So, we only need to verify estimates (2.29) and (2.30). We give below only the formal derivation of that estimates which can be easily justified using, e.g., the Galerkin approximation method (see also Section 4.2 for more details), since now $\Psi_{\delta}$ is smooth.

We start with the dissipative estimate for the $H^{1}$ norm. Recall that (2.4) states that $F(t, \cdot)$ is the potential for $f(t, \cdot)$. The energy estimate gives

$$
\begin{aligned}
& \frac{d}{d \tau}\left(\frac{1}{2}(\mathcal{B} u(\tau), u(\tau))_{L^{2}}+(F(\varepsilon \tau, u(\tau)), 1)_{L^{2}}\right)+1 / 2\left\|\partial_{\tau} u(\tau)\right\|_{L^{2}}^{2} \\
& \quad+\left(\Psi_{\delta}^{\prime}\left(\partial_{\tau} u(\tau)\right), \partial_{\tau} u(\tau)\right)_{L^{2}}=\varepsilon\left(F_{t}^{\prime}(\varepsilon \tau, u(\tau)), 1\right)_{L^{2}},
\end{aligned}
$$

where we used the chain rule

$$
\frac{d}{d \tau}(F(\varepsilon \tau, u(\tau)), 1)_{L^{2}}=\left(f_{\varepsilon}(\tau, u(\tau)), \partial_{\tau} u(\tau)+\varepsilon\left(F_{t}^{\prime}(\varepsilon \tau, u(\tau)), 1\right)_{L^{2}} .\right.
$$

Furthermore, we multiply equation (2.27) by $u(\tau)$ and integrate over $x$. Then, using (2.2) and the boundedness of $v \mapsto \Psi_{\delta}^{\prime}(v)$, see Lemma A.2, we have

$$
\frac{d}{d \tau}\|u(\tau)\|_{L^{2}}^{2}+\kappa\|u(\tau)\|_{H^{1}}^{2}+2\left(f_{\varepsilon}(\tau, u(\tau)), u(\tau)\right)_{L^{2}} \leq C .
$$

where $C$ is independent of $\tau, \varepsilon$ and $\delta$. Multiplying this by $2 L$, where $L$ is sufficiently large, adding this to (2.32) and using (2.7) and $\left\|\Psi_{\delta}^{\prime}\right\| \leq C$ we have

$$
\frac{d}{d \tau} E(\tau, u(\tau))+\theta\|u(\tau)\|_{H^{1}}^{2}+\theta\left\|\partial_{\tau} u(\tau)\right\|_{L^{2}}^{2} \leq C_{1}
$$


with $E(\tau, v):=L\|v\|_{L^{2}}^{2}+\frac{1}{2}(\mathcal{B} v, v)_{L^{2}}+(F(\varepsilon \tau, v), 1)_{L^{2}}$ and positive constants $\theta$ and $C_{1}$ independent of $\varepsilon, \delta$ and $\tau$. Due to (2.7), we have $\rho\|v\|_{H^{1}}^{2}-C \leq E(\tau, v) \leq$ $Q\left(\|v\|_{H^{1}}\right)$ for some positive $\rho$ and monotone function $Q$. Applying the Gronwalltype estimate (see [3, Lem.2.7]) to equation (2.33), we obtain

$$
\|u(\tau)\|_{H^{1}}^{2}+\int_{\tau}^{\tau+1}\left\|\partial_{\tau} u(r)\right\|_{L^{2}}^{2} \mathrm{~d} r \leq Q\left(\|u(0)\|_{H^{1}}\right) \mathrm{e}^{-k \tau}+C_{*}
$$

for a proper monotone function $Q$ and positive constants $K$ and $C_{*}$.

As the next step, we verify estimate (2.30). To this end, we differentiate equation (2.27) by $\tau$ and denote $v(\tau)=\partial_{\tau} u(\tau)$. Then, the function $v$ solves

$$
\partial_{\tau} v+\Psi_{\delta}^{\prime \prime}(v) \partial_{\tau} v+\mathcal{B} v+f_{\varepsilon, u}^{\prime}(\tau, u) v+f_{\varepsilon, \tau}^{\prime}(\tau, u)=0
$$

Introducing $P_{\delta}(v)=\Psi_{\delta}^{\prime}(v) . v-\Psi_{\delta}(v)$ we have $\frac{d}{d \tau} P_{\delta}(v)=\Psi_{\delta}^{\prime \prime}(v) \partial_{\tau} v . v$. Hence, multiplication by $v(\tau)$ and integration over $x$ gives

$$
\frac{d}{d \tau}\left[\left(P_{\delta}(v), 1\right)_{L^{2}}+1 / 2\|v\|_{L^{2}}^{2}\right]+(\mathcal{B} v, v)_{L^{2}}+\left(f_{\varepsilon, \tau}^{\prime}(\tau, u)+f_{\varepsilon, u}^{\prime}(\tau, u) v, v\right)_{L^{2}} \leq 0 .
$$

Integrating over $\tau \in\left[\tau_{1}, \tau_{2}\right]$ and using (2.3c) and (A.7), we deduce the desired estimate (2.30). (As already mentioned, in order to justify this calculations, one should first deduce this estimate for the Galerkin approximations and then pass to the $\operatorname{limit} N \rightarrow \infty$.)

Moreover, using estimates (2.8) and (2.10), we deduce from (2.36) that

$$
\frac{d}{d \tau}\left[2\left(P_{\delta}(v), 1\right)_{L^{2}}+\|v\|_{L^{2}}^{2}\right]+\kappa\|v\|_{H^{1}}^{2} \leq C\left(1+\|v\|_{H^{1-\gamma}}^{2}\right) .
$$

Applying the Gronwall estimate to this relation and using (A.7), which gives $\left|P_{\delta}(v)\right| \leq C \delta$, the interpolation estimate $\|\cdot\|_{H^{1-\gamma}} \leq C\|\cdot\|_{L^{2}}^{\gamma}\|\cdot\|_{H^{1}}^{1-\gamma}$, and (2.34) for estimating the integral over the $L^{2}$ norm of $v$, we find

$$
\|v(\tau)\|_{L^{2}}^{2}+\int_{\tau}^{\tau+1}\|v(r)\|_{H^{1}}^{2} \mathrm{~d} r \leq C\|v(0)\|_{L^{2}}^{2} \mathrm{e}^{-k \tau}+C_{*},
$$

where $k$ and $C_{*}$ are independent of $\delta, \tau$ and $\varepsilon$. Furthermore, $v(0)=\partial_{t} u(0)$ can be found from

$$
v(0)+\Psi_{\delta}^{\prime}(v(0))=\Theta_{u}(0):=-\mathcal{B} u(0)-f_{\varepsilon}(0, u(0)),
$$

Since $u(0) \in H^{2}(\Omega)$, analogously to Lemma 2.4, we conclude that $\Theta_{u}(0)$ lies in $L^{2}(\Omega)$. Multiplying now equation (2.39) by $v(0)$ and using the monotonicity of $\Psi_{\delta}^{\prime}$, we obtain $\|v(0)\|_{L^{2}} \leq Q\left(\|u(0)\|_{H^{2}}\right)$. Combining this with (2.38) leads to

$$
\|v(\tau)\|_{L^{2}}^{2}+\int_{\tau}^{\tau+1}\|v(r)\|_{H^{1}}^{2} \mathrm{~d} r \leq Q\left(\|u(0)\|_{H^{2}}\right) \mathrm{e}^{-k \tau}+C_{*},
$$


where the constant $C_{*}$ and monotone function $Q$ are independent of $T$ and $\varepsilon$. Together with (2.17), this provides the desired control of the $H^{2}$ norm of $u$ and finishes the proof of the dissipative estimate (2.29).

Thus, we only need to verify (2.31). To this end, we multiply equation (2.35) by $\partial_{\tau} v(\tau)$ and integrate over $x$. Then, using that $\Psi_{\delta}$ is convex, we conclude that

$$
\left\|\partial_{\tau} v(\tau)\right\|_{L^{2}}^{2}+\frac{d}{d \tau}(\mathcal{B} v(\tau), v(\tau))_{L^{2}} \leq\left\|f_{\varepsilon, u}^{\prime}(\tau, u) v\right\|_{L^{2}}^{2}+\left\|f_{\varepsilon, \tau}^{\prime}(\tau, u)\right\|_{L^{2}}^{2} .
$$

Moreover, due to (2.11b) and (2.11c), we have

$$
\left\|f_{\varepsilon, u}^{\prime}(\tau, u) v\right\|_{L^{2}} \leq Q\left(\|u\|_{H^{2}}\right)\|v\|_{H^{1}}, \quad\left\|f_{\varepsilon, \tau}^{\prime}(\tau, u)\right\|_{L^{2}} \leq Q\left(\|u\|_{H^{2}}\right),
$$

where the function $Q$ is independent of $\varepsilon, \tau, u$ and $v$. Multiplying now estimate (2.41) by $\left(\tau-\tau_{1}\right)$ and integrating over $\tau \in\left(\tau_{1}, \tau_{2}\right)$, we arrive at

$$
\begin{aligned}
\left(\tau_{2}-\tau_{1}\right)\left\|v\left(\tau_{2}\right)\right\|_{H^{1}}^{2} & +\int_{\tau_{1}}^{\tau_{2}}\left(\tau-\tau_{1}\right)\left\|\partial_{\tau} v(\tau)\right\|_{L^{2}}^{2} d \tau \\
& \leq Q\left(\|u\|_{L^{\infty}\left(\left[\tau_{1}, \tau_{2}\right], H^{2}\right)}\right)\left(\tau_{2}-\tau_{1}+1\right) \int_{\tau_{1}}^{\tau_{2}}\left\|\partial_{\tau} u(\tau)\right\|_{H^{1}}^{2} \mathrm{~d} \tau
\end{aligned}
$$

which together with estimate (2.6) give (2.31) and finishes the proof of Theorem 2.8.

Our next task is to construct a strong solution of (2.12) by passing to the limit $\delta \rightarrow 0$ in equations (2.27).

Theorem 2.9. Let the above assumptions hold and let $u_{0} \in H^{2}(\Omega)$. Then, problem (2.12) possesses a (unique) strong solution $u$ in the sense of Definition 2.6. This solution satisfies the energy equality (2.18) and higher energy estimate (2.30) (with $\delta=0$ ). Moreover, $u$ also satisfies the dissipative estimates (2.29) and (2.31) uniformly with respect to $\varepsilon \rightarrow 0$.

Proof. We will construct the required solution $u$ of problem (2.12) as a limit $\delta \rightarrow 0$ of the solutions $u_{\delta}$ of the regularized problems (2.27). Indeed, using the dissipative estimate (2.29), we see that the sequence $u_{\delta}$ is uniformly bounded in $L^{\infty}\left(\mathbb{R}_{+}, H^{2}(\Omega)\right)$ and the corresponding sequence of derivatives $\partial_{\tau} u_{\delta}$ is uniformly bounded in $L^{\infty}\left([0, R], L^{2}(\Omega)\right) \cap L^{2}\left([0, R], H_{0}^{1}(\Omega)\right)$, for every $R>0$. Thus, choosing a subsequence $\left(u_{\delta_{n}}\right)_{n \in \mathbb{N}}$ with $\delta_{n} \rightarrow 0$ we can assume that there exists a weak limit $u=\lim _{n \rightarrow \infty} u_{\delta_{n}}$ such that

$u_{n} \rightarrow u$ weak ${ }^{*}$ in $L^{\infty}\left([0, R], H^{2}(\Omega)\right) \cap W^{1, \infty}\left([0, R], L^{2}(\Omega)\right) \cap H^{1}\left([0, R], H_{0}^{1}(\Omega)\right)$.

Moreover, due to estimate (2.31), we may additionally assume that

$$
u_{n} \rightarrow u \text { weakly* in } W_{l o c}^{1, \infty}\left((0, R], H^{1}(\Omega)\right) \cap H_{l o c}^{2}\left((0, R], L^{2}(\Omega)\right) .
$$


It remains to prove that $u$ solves (2.12). To this end, we set

$$
v_{n}:=\partial_{\tau} u_{n}, \quad \Theta_{n}:=\Theta_{u_{n}}=\Psi_{\delta_{n}}^{\prime}\left(v_{n}\right)+v_{n}
$$

Since the sequence $\partial_{t} u_{n}$ is uniformly bounded in $L^{\infty}\left([0, R], L^{2}(\Omega)\right)$ and $\Psi_{\delta}^{\prime}$ is bounded in $L^{\infty}$, the sequence $\Theta_{n}$ is uniformly bounded in $L^{\infty}\left([0, R], L^{2}(\Omega)\right)$. Consequently, without loss of generality, we can assume that $\Theta_{n} \rightarrow \Theta$ weakly* in that space. We need to prove

$$
\text { (a) } \Theta=\Theta_{u}:=\mathcal{B} u-f_{\varepsilon}(\tau, u), \quad \text { (b) } \Theta \in \partial \Psi(v)+v \text {, }
$$

where $v=\partial_{\tau} u$. Furthermore, the first equality is obvious. Indeed, the operator $\mathcal{B}$ is linear and, thus, $\mathcal{B} u_{n} \rightarrow \mathcal{B} u$ weakly* in $L^{\infty}\left([0, R], L^{2}(\Omega)\right)$. In order to pass to the limit in the term $f_{\varepsilon}\left(\tau, u_{n}\right)$, it is sufficient to note that the weak convergence (2.42) implies the strong convergence

$$
u_{n} \rightarrow u \text { strongly in } C\left([0, R], H^{1-\gamma}(\Omega)\right)
$$

for any $\gamma>0$ and use the first assertion of Lemma 2.1 to conclude that $f_{\varepsilon}\left(u_{n}\right) \rightarrow$ $f_{\varepsilon}(u)$ in $C\left([0, R], H^{-1}\right)$, and $(2.45)($ a) is established.

To check (2.45)(b) let $A_{\delta}(w):=\Psi_{\delta}(w)+1 / 2\|w\|^{2}(c f$. (A.12)). Then, multiplying (2.27) by $v_{n}$, we find that, analogously to (2.18),

$$
\begin{aligned}
& \left\langle A_{\delta_{n}}^{\prime}\left(v_{n}\right), v_{n}\right\rangle>_{R}+\frac{1}{2}\left(\mathcal{B} u_{n}(R), u_{n}(R)\right)_{L^{2}} \\
& \left.\quad=\frac{1}{2}(\mathcal{B} u(0)), u(0)\right)_{L^{2}}-\left\langle f_{\varepsilon}\left(\tau, u_{n}\right), v_{n}\right\rangle>_{R},
\end{aligned}
$$

where by $\langle\cdot, \cdot\rangle_{R}$ we denote the scalar product in $L^{2}([0, R] \times \Omega)$ and $v_{n}:=\partial_{\tau} u_{n}$. Passing now to the limit $n \rightarrow \infty$ in that relation and using that $f_{\varepsilon}\left(u_{n}\right) \rightarrow f_{\varepsilon}(u)$ strongly in $C\left([0, R], H^{-1}\right)$ and that $\mathcal{B}$ is positive definite, we infer

$$
\begin{aligned}
\limsup _{n \rightarrow \infty}\left\langle A_{\delta_{n}}^{\prime}\left(v_{n}\right), v_{n}\right\rangle_{R} \leq & \frac{1}{2}(\mathcal{B} u(0), u(0))_{L^{2}}-\frac{1}{2}(\mathcal{B} u(R), u(R))_{L^{2}} \\
& -\left\langle f_{\varepsilon}(\tau, u), v\right\rangle_{R} .
\end{aligned}
$$

Analogously, multiplying relation $(2.45)$ (a) by $v$ and integrating over $(\tau, x)$, we get

$$
\langle\Theta, v\rangle_{R}=\frac{1}{2}(\mathcal{B} u(0), u(0))_{L^{2}}-\frac{1}{2}(\mathcal{B} u(R), u(R))_{L^{2}}-\left\langle f_{\varepsilon}(\tau, u), v\right\rangle_{R},
$$

which, together with (2.48), gives

$$
\limsup _{n \rightarrow \infty}\left\langle A_{\delta_{n}}^{\prime}\left(v_{n}\right), v_{n}\right\rangle_{R} \leq\langle\Theta, v\rangle_{R} .
$$


Let us now consider the convex functional

$$
\mathcal{A}_{\delta}(w):=\int_{(\tau, x) \in[0, R] \times \Omega} A_{\delta}(w(\tau, x)) \mathrm{d} \tau \mathrm{d} x
$$

on the space $L^{2}([0, R] \times \Omega)$. Then, $(2.45)(\mathrm{b})$ means that $\Theta \in \partial \mathcal{A}_{0}(v)$. Let now $(w, z) \in\left[L^{2}([0, R] \times \Omega)\right]^{2}$ with $z \in \partial \mathcal{A}_{0}(w)$ be an arbitrary point of the graph of maximal monotone operator $\partial \mathcal{A}_{0}$. Then, due to Lemma A.3, there exists a sequence $w_{n}$ strongly convergent in $L^{2}([0, R] \times \Omega)$ to $w$ such that $\mathcal{A}_{\delta_{n}}^{\prime}\left(w_{n}\right)=z$. Since $\mathcal{A}_{\delta_{n}}^{\prime}$ is monotone, we have

$$
\left\langle\mathcal{A}_{\delta_{n}}^{\prime}\left(v_{n}\right)-z, v_{n}-w_{n}\right\rangle_{R}=\left\langle\mathcal{A}_{\delta_{n}}^{\prime}\left(v_{n}\right)-\mathcal{A}_{\delta_{n}}^{\prime}\left(w_{n}\right), v_{n}-w_{n}\right\rangle_{R} \geq 0 .
$$

Passing to the limit $n \rightarrow \infty$ in (2.51) and using (2.50), we finally have

$$
\langle\Theta-z, v-w\rangle_{R} \geq 0, \quad w \in L^{2}([0, R] \times \Omega), \quad z \in \partial \mathcal{A}_{0}(w) .
$$

Since $(z, w)$ is an arbitrary point in the graph, maximal monotonicity of the subdifferential $\partial \mathcal{A}_{0}$ implies $\Theta \in \partial \mathcal{A}_{0}(v)$. Thus, (2.45)(b) holds, and $u$ solves the limit problem (2.12).

We are now ready to check the remaining assertions of the theorem. Indeed, the weak convergence (2.42) and (2.43) is sufficient to pass to the limit $n \rightarrow \infty$ in the dissipative estimates (2.29) and (2.31) for $u_{n}$ and the energy equality for $u$ was verified in Corollary 2.5 . To check the remaining higher energy estimate (2.30), we need a strong convergence, namely

$$
\partial_{\tau} u_{n} \rightarrow \partial_{\tau} u \text { strongly in } L^{2}\left([0, R], L^{2}(\Omega)\right) .
$$

To this end, we rewrite the energy equality (2.18) in the form

$$
\begin{aligned}
2\left\langle\Psi\left(v_{n}\right), 1\right\rangle_{R}+2\left\langle\left|v_{n}\right|^{2}, 1\right\rangle_{R}+\left(\mathcal{B} u_{n}(R), u_{n}(R)\right)_{L^{2}}-(\mathcal{B} u(0), u(0))_{L^{2}} \\
=2\left\langle\Psi_{\delta_{n}}\left(v_{n}\right)-\Psi\left(v_{n}\right), 1\right\rangle_{R}-2\left\langle P_{\delta_{n}}\left(v_{n}\right), 1\right\rangle_{R}-2\left\langle f_{\varepsilon}\left(\tau, u_{n}\right), v_{n}\right\rangle_{R} .
\end{aligned}
$$

Using the fact that $f_{\varepsilon}$ is relatively compact and estimates (A.4) and (A.7), passing to the limit $n \rightarrow \infty$ gives

$$
\begin{aligned}
& \left.\lim _{n \rightarrow \infty}\left[\left\langle 2 \Psi\left(v_{n}\right)+2\left|v_{n}\right|^{2}, 1\right\rangle_{R}+\left(\mathcal{B} u_{n}(R)\right), u_{n}(R)\right)_{L^{2}}\right] \\
& \quad=(\mathcal{B} u(0), u(0))_{L^{2}}-2\left\langle f_{\varepsilon}(\tau, u), v\right\rangle_{R} .
\end{aligned}
$$

Together with the energy equality for the limit equation (2.12) gives

$$
\begin{gathered}
\lim _{n \rightarrow \infty}\left[\left\langle 2 \Psi\left(v_{n}\right)+2\left|v_{n}\right|^{2}, 1\right\rangle_{R}+\left(\mathcal{B} u_{n}(R), u_{n}(R)\right)_{L^{2}}\right] \\
=\left\langle 2 \Psi(v)+2|v|^{2}, 1\right\rangle_{R}+(\mathcal{B} u(R), u(R))_{L^{2} .}
\end{gathered}
$$


Since the operators $\Psi$ and $\mathcal{B}$ are convex, this implies

$$
\lim _{n \rightarrow \infty}\left\langle\left|v_{n}\right|^{2}, 1\right\rangle_{R}=\left\langle|v|^{2}, 1\right\rangle_{R} \text { and } \lim _{n \rightarrow \infty}\left(\mathcal{B} u_{n}(R), u_{n}(R)\right)_{L^{2}}=(\mathcal{B} u(R), u(R))_{L^{2}}
$$

Since $L^{2}([0, R] \times \Omega)$ is uniformly convex, we deduce the strong convergence (2.52).

It is now not difficult to pass to the limit in (2.30). Indeed, the strong convergence (2.52) together with weak convergence (2.42) imply that $\partial_{\tau} u_{n} \rightarrow \partial_{\tau} u$ strongly in $L^{2}\left([0, R], W^{1-\gamma}(\Omega)\right)$, for every $\gamma>0$ and $\left\|\partial_{\tau} u_{n}(\tau)\right\|_{L^{2}} \rightarrow\left\|\partial_{\tau} u(\tau)\right\|_{L^{2}}$ for almost all $\tau \in \mathbb{R}_{+}$. These convergence, together with assertions 1) and 3) of Lemma 2.1 and convergence (2.46) allow us to pass to the limit $\delta \rightarrow 0$ in (2.30) and show that the higher energy estimate holds for almost all $0 \leq \tau_{1} \leq \tau_{2}$ (including $\tau_{1}=0$ ). In order to see that this estimate actually holds for all $\tau_{1}$ and $\tau_{2}$, it is sufficient to recall that, due to estimate $(2.31), \partial_{\tau}^{2} u \in L^{2}([0, R] \times \Omega)$ and, consequently, the function $\tau \mapsto\left\|\partial_{\tau} u(\tau)\right\|_{L^{2}}^{2}$ is continuous (and even absolutely continuous) on $[0, R]$. Thus, Theorem 2.9 is proved.

\subsection{Rate-independent a priori estimates}

Our next task now is to deduce several crucial estimates for $\partial_{\tau} u(\tau)$ based on the energy equality and the higher order energy estimate (2.30). The emphasis here is to obtain estimates that are suitable for the vanishing-viscosity limit, i.e., they have be uniform for $\varepsilon \rightarrow 0$. We start from the dissipation integral for solutions of problem (2.12).

Corollary 2.10. Let the assumptions of Theorem 2.9 hold and let $u$ be a solution of problem (2.12). Then, for every $\tau_{1}, \tau_{2} \in \mathbb{R}_{+}, \tau_{2} \geq \tau_{1}$, one has

$$
\int_{\tau_{1}}^{\tau_{2}}\left\|\partial_{\tau} u(\tau)\right\|_{L^{1}(\Omega)}+\left\|\partial_{\tau} u(\tau)\right\|_{L^{2}}^{2} \mathrm{~d} \tau \leq Q\left(\|u(0)\|_{H^{2}(\Omega)}\right)\left[1+\varepsilon\left(\tau_{2}-\tau_{1}\right)\right],
$$

where the monotone function $Q$ is independent of $\varepsilon, \tau_{1}$ and $\tau_{2}$.

Proof. Estimate (2.53) follows from energy equality (2.18) and the uniform bounds for the $H^{2}$-norm of the solution obtained above. Indeed, using (2.18) together with (2.29), (2.9) and the obvious equality $f_{\varepsilon}(\tau, u) . \partial_{\tau} u=\partial_{\tau} F(\varepsilon \tau, u)-\varepsilon F_{t}^{\prime}(\varepsilon \tau, u)$, we get

$$
\int_{\tau_{1}}^{\tau_{2}} \widetilde{\Psi}\left(\partial_{\tau} u(\tau)\right)+\frac{1}{2}\left\|\partial_{\tau} u(\tau)\right\|_{L^{2}}^{2} \mathrm{~d} \tau \leq Q\left(\left\|u_{0}\right\|_{H^{1}}\right)+\int_{\tau_{1}}^{\tau_{2}} \varepsilon\left(F_{t}^{\prime}(\varepsilon \tau, u(\tau)), 1\right)_{L^{2}} \mathrm{~d} \tau .
$$

Using estimates (2.9) and (2.29) in order to estimate the integral with $F_{t}^{\prime}$ and estimate (2.3)(1) and for estimating the term with $\Psi$, we obtain the required estimate (2.53) and finish the proof of Corollary 2.10. 
We formulate now the $L^{1}\left([0, R], H^{1}\right)$-estimate for the derivative $\partial_{\tau} u$ which is crucial for what follows. The important structure here is that the $L^{1}$ norm with respect to $t \in[0, T]$ is scaling invariant. In fact, it would be enough to control $\int_{\tau_{1}}^{\tau_{2}}\left\|\partial_{\tau} u(\tau)\right\|_{L^{2}} \mathrm{~d} \tau$, but we state the stronger result in terms of the $H^{1}$ norm, as this improvement comes for free from the parabolic regularity theory. The crucial interpolation estimate is

$$
\|v\|_{L^{2}} \leq C \tilde{\Psi}(v)^{\gamma}\|v\|_{H^{1}}^{1-\gamma} \quad \text { for all } v \in H^{1}(\Omega) .
$$

Since $\widetilde{\Psi}$ is bounded from below by a multiple of the norm in $L^{1}(\Omega)$, we obtain this with $\gamma=2 /(n+2)$ from standard Gagliardo-Nirenberg estimates.

Proposition 2.11. Let the assumptions of Section 2.1 hold and let $u=u(\tau)$ be a solution of problem (2.12) in the sense of Definition 2.6. Then, the following estimate holds:

$$
\int_{\tau_{1}}^{\tau_{2}}\left\|\partial_{\tau} u(\tau)\right\|_{H^{1}} \mathrm{~d} \tau \leq C\left(1+\varepsilon\left(\tau_{2}-\tau_{1}\right)\right)
$$

where $C>0$ depends on $\|u(0)\|_{H^{2}}$, but is independent of $\varepsilon>0, \tau_{1} \geq 0$, and $\tau_{2}>\tau_{1}$.

Proof. As we will see, the desired estimate is a corollary of estimate (2.30) and the dissipation integral (2.53). Indeed, using $f_{\varepsilon, \tau}^{\prime}(\tau, u)=\varepsilon f_{t}^{\prime}(\varepsilon \tau, u),(2.8),(2.10)$, and the dissipative estimate (2.29), one can transform estimate (2.30) into

$\left\|v\left(\tau_{2}\right)\right\|_{L^{2}}^{2}-\left\|v\left(\tau_{1}\right)\right\|_{L^{2}}^{2}+\kappa \int_{\tau_{1}}^{\tau_{2}}\|v(s)\|_{H^{1}}^{2} \mathrm{~d} s \leq C \int_{\tau_{1}}^{\tau_{2}}\left(\varepsilon^{2}+\|v(s)\|_{L^{2}}^{2}\right) \mathrm{d} s$,

where $v=\partial_{\tau} u$ and $C$ depends only on $\|u(0)\|_{H^{2}}$. Moreover, according to Lemma B.1, for every function $\phi \in C^{1}\left(\mathbb{R}_{+}\right)$, we have

$$
\begin{gathered}
\phi\left(\tau_{2}\right)\left\|v\left(\tau_{2}\right)\right\|_{L^{2}}^{2}-\phi\left(\tau_{1}\right)\left\|v\left(\tau_{1}\right)\right\|_{L^{2}}^{2}+\kappa \int_{\tau_{1}}^{\tau_{2}} \phi(r)\|v(r)\|_{H^{1}}^{2} \mathrm{~d} r \\
\leq C \int_{\tau_{1}}^{\tau_{2}}\left[\phi(r)+\phi^{\prime}(r)\right]\left(\varepsilon^{2}+\|v(r)\|_{L^{2}}^{2}\right) \mathrm{d} r .
\end{gathered}
$$

We now fix $\tau_{1}=\tau, \tau \leq \tau_{2} \leq \tau+2$ and $\phi(r)=\phi_{\tau}(r):=(\tau-r)^{N}$ for $\tau \geq r$ and $\phi(r)=0$ for $\tau \leq r$ where $N>1$ is a sufficiently large exponent which will be fixed below. Then, this function satisfies the estimate

$$
\left|\phi^{\prime}(r)\right| \leq N \phi(r)^{\delta_{N}} \quad \text { with } \delta_{N}:=\frac{N-1}{N}
$$


This and the interpolation estimate (2.54) allow us to estimate the integral in the right-hand side of (2.57) via

$$
\begin{aligned}
& \int_{\tau}^{\tau_{2}}\left[\phi(r)+\phi^{\prime}(r)\right]\|v(r)\|_{L^{2}}^{2} \mathrm{~d} r \leq C_{N} \int_{\tau}^{\tau_{2}} \phi^{\delta_{N}}(r)\|v(r)\|_{L^{2}}^{2} \mathrm{~d} r \\
& \leq C_{N}^{\prime} \int_{\tau}^{\tau_{2}}\left(\phi(r)^{1 / 2}\|v(r)\|_{H^{1}}\right)^{2-2 \gamma}\left(\phi(r)^{\beta / 2}\|v(r)\|_{L^{1}(\Omega)}\right)^{2 \gamma} \mathrm{d} r \\
& \quad \leq C_{N}^{\prime}\left(\int_{\tau}^{\tau_{2}} \phi(r)\|v(r)\|_{H^{1}}^{2} \mathrm{~d} r\right)^{1-\gamma}\left(\int_{\tau}^{\tau_{2}} \phi(r)^{\beta}\|v(r)\|_{L^{1}(\Omega)}^{2} \mathrm{~d} r\right)^{\gamma} \\
& \quad \leq \frac{\kappa}{2 C} \int_{\tau}^{\tau_{2}} \phi(r)\|v(r)\|_{H^{1}}^{2} \mathrm{~d} r+C_{N}^{\prime \prime} \int_{\tau}^{\tau_{2}} \phi(r)^{\beta}\|v(r)\|_{L^{1}}^{2} \mathrm{~d} r,
\end{aligned}
$$

where $\beta=\left(\delta_{N}-1+\gamma\right) / \gamma=1-\left(1-\delta_{N}\right) / \gamma$. Clearly, $\beta<1$, but we may fix $N$ such that $\beta$ remains positive. Fixing now $N=\frac{2}{\gamma}$ gives $\beta=\frac{1}{2}$ and (2.57) takes the form

$$
\phi\left(\tau_{2}\right)\left\|v\left(\tau_{2}\right)\right\|_{L^{2}}^{2}+\int_{\tau}^{\tau_{2}} \phi(r)\|v(r)\|_{H^{1}}^{2} \mathrm{~d} r \leq C\left(\varepsilon^{2}+\int_{\tau}^{\tau_{2}} \phi(r)^{1 / 2}\|v(r)\|_{L^{1}(\Omega)}^{2} \mathrm{~d} r\right) .
$$

Furthermore, we estimate the integral in the right-hand side via

$$
\begin{aligned}
\int_{\tau}^{\tau_{2}} \phi(r)^{1 / 2}\|v(r)\|_{L^{1}(\Omega)}^{2} \mathrm{~d} r & \leq C \sup _{r \in\left[\tau, \tau_{2}\right]}\left\{\phi(r)^{1 / 2}\|v(r)\|_{L^{2}}\right\} \int_{\tau}^{\tau_{2}}\|v(r)\|_{L^{1}} \mathrm{~d} r \\
& \leq 1 /\left(2 C \sup _{r \in\left[\tau, \tau_{2}\right]}\left\{\phi(r)\left\|\partial_{\tau} u(r)\right\|_{L^{2}}^{2}\right\}+C_{1}\left(\int_{\tau}^{\tau_{2}}\|v(r)\|_{L^{1}} \mathrm{~d} r\right)^{2} .\right.
\end{aligned}
$$

Inserting this estimate into (2.58) with $\tau_{2}=\tau+2$, we have

$$
\int_{\tau}^{\tau+2} \phi(r)\|v(r)\|_{H^{1}}^{2} \mathrm{~d} r \leq C\left(\varepsilon+\int_{\tau}^{\tau+2}\|v(r)\|_{L^{1}(\Omega)} \mathrm{d} r\right)^{2}
$$

Moreover, using $\phi(r) \geq 1$ for $r \geq \tau+1$ we obtain

$$
\left(\int_{\tau+1}^{\tau+2}\|v(r)\|_{H^{1}} \mathrm{~d} r\right)^{2} \leq \int_{\tau+1}^{\tau+2}\|v(r)\|_{H^{1}}^{2} \mathrm{~d} r \leq C \int_{\tau}^{\tau+2} \phi(r)\|v(r)\|_{H^{1}}^{2} \mathrm{~d} r
$$

and conclude

$$
\int_{\tau+1}^{\tau+2}\|v(r)\|_{H^{1}} \mathrm{~d} r \leq C\left(\varepsilon+\int_{\tau}^{\tau+2}\|v(r)\|_{L^{1}(\Omega)} \mathrm{d} r\right),
$$

where the constant $C$ depends on $\|u(0)\|_{H^{2}}$ but not on $\tau \geq 0$ and $\varepsilon>0$. This estimate allows us to estimate the norm of $v(\tau)$ for $\tau \geq 1$. 
For the interval $\tau \in[0,1]$ we argue analogously, but without the usage of the cut-off function $\phi$. Working on (2.56) directly we find

$$
\int_{0}^{1}\|v(r)\|_{H^{1}} \mathrm{~d} r \leq C\left(\varepsilon+\|v(0)\|_{L^{2}}+\int_{0}^{1}\|v(r)\|_{L^{1}(\Omega)} \mathrm{d} r\right) .
$$

To finish the proof we sum the estimates (2.59) with $\tau=\tau_{1}-1, \tau_{1}, \tau_{1}+1, \cdots, \tau_{1}+$ $\left[\tau_{2}-\tau_{1}\right]$. In the case $\tau_{1}<1$ use (2.60) for the first step instead of (2.59). This gives

$$
\int_{\tau_{1}}^{\tau_{2}}\|v(\tau)\|_{H^{1}} \mathrm{~d} \tau \leq C\left(\varepsilon\left(\tau_{2}-\tau_{1}\right)+1+\int_{\max \left\{\tau_{1}-1,0\right\}}^{\tau_{2}}\|v(\tau)\|_{L^{1}(\Omega)} \mathrm{d} \tau\right) .
$$

Employing the dissipation bound (2.53) gives the desired estimate (2.55).

We are now able to return back to the original time $t=\varepsilon \tau$ and the associated equation (2.1). The results obtained from this time rescaling read as follows.

Theorem 2.12. Let the assumptions of Section 2.1 hold. Then, for all $\varepsilon>0$, there exists a unique strong solution $u$ of problem (2.1). This solution satisfies the dissipative estimate

$$
\|u(t)\|_{H^{2}}+\varepsilon\left\|\partial_{t} u(t)\right\|_{L^{2}} \leq Q\left(\|u(0)\|_{H^{2}}\right) \mathrm{e}^{-\alpha t / \varepsilon}+C_{f},
$$

where $\alpha>0, C_{f}>0$, and the monotone function $Q$ are independent of $\varepsilon$ and $t$. Moreover, we have

$$
\int_{T}^{T+1}\left\|\partial_{t} u(t)\right\|_{H^{1}} \mathrm{~d} t \leq C
$$

where $C$ depends on $\|u(0)\|_{H^{2}}$, but not on $\varepsilon$ and $T \geq 0$.

Indeed, estimate (2.62) is an immediate corollary of estimates (2.29) and estimates (2.63) follow from Proposition 2.11 taking into the account that the $L^{1}([0, T], Y)$-norm of $\partial_{t} u$ is scaling invariant, where $Y$ is any Banach space. This means more precisely if $u_{\varepsilon}(t)=\tilde{u}(\varepsilon t)$, then $\int_{0}^{T}\left\|\partial_{t} u_{\varepsilon}(t)\right\|_{Y} \mathrm{~d} t=\int_{0}^{T / \varepsilon}\left\|\partial_{\tau} \tilde{u}(\tau)\right\|_{Y} \mathrm{~d} \tau$.

Remark 2.13. As we see, the possibility to construct a strong solution of problem (2.1) and to verify the crucial estimates (2.62) and (2.63) is strongly based on the validity of the higher energy estimate (2.30) which is a weakened integrated form of the equality (2.22). The method of proving (2.30) given above does not allow to obtain the higher energy equality (2.22) since we do not know how to verify the strong convergence of $\partial_{\tau} u_{n}$ in $L^{2}\left([0, R], H^{1}(\Omega)\right)$ in Theorem 2.9 (and even earlier, in the proof of the existence of approximating solution via the Galerkin method). By this reason, the equality may be lost under the passage to the limit and only the higher energy estimate can be obtained.

We will see in Section 3, using an alternative direct method which does not require the approximations, that the higher energy equality (2.22) holds for a.a. $\tau>0$. However, it seems difficult to obtain (2.30) directly from (2.22) since the 
function $\tau \mapsto \partial_{\tau} u(\tau) \in L^{2}(\Omega)$ is continuous for $\tau>0$ only, and we do not know how to verify even the weak continuity $\partial_{\tau} u \in C_{w}\left([0, R], L^{2}\right)$ at $\tau=0$. By this reason, we cannot deduce (2.30) with $S=0$ directly from (2.22) and some approximations are still required, see Section 3 for more details.

Remark 2.14. We also note that assumption (2.4) on the gradient structure of the non-linearity $f$ can be somehow relaxed. Indeed, it is not difficult to see that this assumption has been used only in order to prove the dissipative estimate (2.53). Thus, if this estimate is a priori known by some other arguments, then all of the results of the paper will hold even without the gradient assumption (2.4). In particular, if the nonlinearity $f$ has the structure

$$
f(t, u)=f_{0}(t, u)+f_{1}(t, u)
$$

where $f_{0}$ is gradient and satisfies all of the assumptions of Section 2.1 and the function $f_{1}$ is uniformly bounded and subordinated to $\partial \Psi$, i.e.

$$
\left|f_{1}(t, u)\right| \leq \beta<\beta_{1}
$$

where $\beta_{1}$ is as in (2.3a). Then the dissipative estimate (2.53) holds, since we have

$$
\int_{\tau_{1}}^{\tau} \Psi\left(\partial_{\tau} u(\tau)\right) \mathrm{d} \tau+\int_{\tau_{1}}^{\tau_{2}}\left(f_{1}(\varepsilon \tau, u), \partial_{\tau} u\right)_{L^{2}} \mathrm{~d} \tau \geq\left(\beta_{1}-\beta\right) \int_{\tau_{1}}^{\tau_{2}}\left\|\partial_{\tau} u(\tau)\right\|_{L^{1}(\Omega)} \mathrm{d} \tau
$$

and (2.53) can be obtained exactly as in the gradient case. Thus, all of the results of the paper remain true in the slightly non-gradient case (2.64).

\subsection{The vanishing-viscosity limit}

The aim of this section is to clarify the limiting behavior of the solutions $u_{\varepsilon}(t)$ of (2.1) for $\varepsilon \rightarrow 0$. We follow the approach in [10] (see also $[19,20]$ ) where solutions are considered as curves in the extended state space $[0, T] \times L^{2}(\Omega)$. Thus we hope to understand the convergence of the whole graph rather than the function only. In order to do so, we introduce an arc-length parametrization via the scaled time variable

$$
s(t):=t+\int_{0}^{t}\left\|\partial_{t} u_{\varepsilon}(r)\right\|_{L^{2}} \mathrm{~d} r
$$

where $u_{\varepsilon}(t)$ solves (2.1). Then, $\frac{d s}{d t}=1+\left\|\partial_{t} u_{\varepsilon}(t)\right\|_{L^{2}}$ and, due to estimate (2.62), we have

$$
1 \leq \frac{d s}{d t} \leq C / \varepsilon
$$

where the constant $C$ depends only on the initial data. Thus, we can define the inverse function $t_{\varepsilon}(s)$ to (2.66) and rewrite equation (2.1) with respect to new dependent variable $\tilde{u}_{\varepsilon}(s):=u_{\varepsilon}\left(t_{\varepsilon}(s)\right)$. To simplify the notations, we will write again $u_{\varepsilon}(s)$ instead of $\tilde{u}_{\varepsilon}(s)$, since keeping the argument $s$ indicates that we are dealing 
with the arc-length parametrization. As a consequence we are now looking for a pair $\left(u_{\varepsilon}, t_{\varepsilon}\right):[0, S] \rightarrow L^{2}(\Omega) \times \mathbb{R}$ which has to satisfy the problem

$$
\begin{aligned}
& \partial \Psi\left(\partial_{s} u_{\varepsilon}(s)\right)+\frac{\varepsilon}{1-\left\|\partial_{s} u_{\varepsilon}(s)\right\|_{L^{2}}} \partial_{s} u_{\varepsilon}(s)+\mathcal{B} u_{\varepsilon}(s)+f\left(t_{\varepsilon}(s), u_{\varepsilon}(s)\right) \ni 0, \\
& t_{\varepsilon}^{\prime}(s)+\left\|\partial_{s} u_{\varepsilon}(s)\right\|_{L^{2}}=1, \\
& u_{\varepsilon}(0)=u_{0}, \quad t_{\varepsilon}(0)=0 .
\end{aligned}
$$

In particular, again due to (2.62), we have

$$
\left\|\partial_{s} u(s)\right\|_{L^{2}}=\left\|\partial_{t} u_{\varepsilon}(t)\right\|_{L^{2}}\left(\frac{d s}{d t}\right)^{-1}=\frac{\left\|\partial_{t} u_{\varepsilon}(t)\right\|_{L^{2}}}{1+\left\|\partial_{t} u_{\varepsilon}(t)\right\|_{L^{2}}} \leq \frac{C}{C+\varepsilon}<1 .
$$

Consequently, for $\varepsilon>0$ equation (2.68) is fully equivalent to (2.1) if the corresponding rescalings are performed.

Following [10] we introduce the convex functional $\mathcal{C}_{\varepsilon}: L^{2}(\Omega) \rightarrow \mathbb{R}_{+}$via

$$
\mathcal{C}_{\varepsilon}(v):= \begin{cases}\varepsilon\left(\ln \left(\frac{1}{1-\|v\|_{L^{2}}}\right)-\|v\|_{L^{2}}\right) & \text { for }\|v\|_{L^{2}}<1 \\ \infty & \text { for }\|v\|_{L^{2}} \geq 1\end{cases}
$$

A simple calculation shows that

$$
\partial \mathcal{C}_{\varepsilon}(v)= \begin{cases}\frac{\varepsilon}{1-\|v\|_{L^{2}}} v & \text { for }\|v\|_{L^{2}}<1 \\ \varnothing & \text { for }\|v\|_{L^{2}} \geq 1\end{cases}
$$

Thus, system (2.68) can be rewritten in the following equivalent form:

$$
\left\{\begin{array}{l}
\partial \Psi\left(\partial_{s} u_{\varepsilon}(s)\right)+\partial \mathcal{C}_{\varepsilon}\left(\partial_{s} u_{\varepsilon}(s)\right)+\mathcal{B} u_{\varepsilon}(s)+f\left(t_{\varepsilon}(s), u_{\varepsilon}(s)\right) \ni 0, \\
t_{\varepsilon}^{\prime}(s)+\left\|\partial_{s} u_{\varepsilon}(s)\right\|_{L^{2}}=1 \\
u_{\varepsilon}(0)=u_{0}, \quad t_{\varepsilon}(0)=0
\end{array}\right.
$$

It is easy to see that the functionals $\mathcal{C}_{\varepsilon}$ converge to $\mathcal{C}_{0}$ given by

$$
\mathcal{C}_{0}(v):= \begin{cases}0 & \text { for }\|v\|_{L^{2}} \leq 1 \\ \infty & \text { for }\|v\|_{L^{2}}>1\end{cases}
$$

Moreover, $\partial \mathcal{C}_{\varepsilon}$ converges to the limit operator $\partial \mathcal{C}_{0}$, see Appendix A. Thus, we can expect that a subsequence of the solutions $\left(u_{\varepsilon}, t_{\varepsilon}\right)$ converge, in a suitable sense, to the solution $(u, t)$ of the limit problem

$$
\left\{\begin{array}{l}
\partial \Psi\left(\partial_{s} u(s)\right)+\partial \mathcal{C}_{0}\left(\partial_{s} u(s)\right)+\mathcal{B} u(s)+f(t(s), u(s)) \ni 0 \\
t^{\prime}(s)+\left\|\partial_{s} u(s)\right\|_{L^{2}}=1 \\
u(0)=u_{0}, \quad t(0)=0 .
\end{array}\right.
$$

The rest of the current subsection is devoted to a rigorous justification of the passage from (2.72) to (2.74). We start with a control of new time $s=s_{\varepsilon}(t)$, which is uniform with respect to $\varepsilon \rightarrow 0$. 
Lemma 2.15. Let the assumptions of Section 2.1 hold and let $u=u_{\varepsilon}(t)$ is a strong solution of problem (2.1). Then, the scaled time $t_{\varepsilon}(s)$ satisfies estimate:

$$
s \geq t_{\varepsilon}(s) \geq s / C-C, \quad t_{\varepsilon}(0)=0, \quad t_{\varepsilon}^{\prime}(s) \geq 0,
$$

for some positive constant $C$, which is independent of $\varepsilon$.

Indeed, estimate (2.75) follows immediately from the definition (2.66) of the scaled time $s$ and the uniform estimate (2.63) for the integral of $\partial_{t} u(t)$. The next lemma interprets the uniform estimates obtained in the previous section in terms of the solutions $\left(u_{\varepsilon}(s), t_{\varepsilon}(s)\right)$ of problem $(2.72)$.

Lemma 2.16. Let the above conditions hold. Then, the solution $\left(u_{\varepsilon}, t_{\varepsilon}\right)$ of problem (2.72) satisfy the following estimates:

$$
\begin{array}{ll}
\left\|u_{\varepsilon}(s)\right\|_{H^{2}} \leq C=C\left(\left\|u_{0}\right\|_{H^{2}}\right), & \int_{S}^{S+1}\left\|\partial_{s} u_{\varepsilon}(s)\right\|_{H^{1}} \mathrm{~d} s \leq C, \\
s \geq t_{\varepsilon}(s) \geq s / C-C, & 1 \geq t_{\varepsilon}^{\prime}(s) \geq 0,
\end{array}
$$

where $C$ is independent of $\varepsilon \rightarrow 0, s \geq 0$ and $S \geq 0$.

Indeed, first estimate of (2.76) is an immediate corollary of (2.62) and the scaling invariance of the $L^{\infty}$ norm. Analogously, the second estimate of (2.76) follows from (2.63) and the scaling invariance of the $L^{1}$-norm of the time derivative. Finally, the third one is just repeats estimate (2.75). We are now ready to pass to the limit $\varepsilon \rightarrow 0$ and formulate the main result of this section.

Theorem 2.17. Let the assumptions of Section 2.1 hold and for $\varepsilon>0$ let $\left(u_{\varepsilon}, t_{\varepsilon}\right)$ : $[0, S] \rightarrow L^{2}(\Omega) \times \mathbb{R}$ be solutions of problem (2.72) as constructed above. Then, there exists a sequence $\varepsilon_{n} \rightarrow 0$ such that the associated solutions $\left(u_{n}(s), t_{n}(s)\right):=$ $\left(u_{\varepsilon_{n}}(s), t_{\varepsilon_{n}}(s)\right)$ tend to the limit pair $(u(s), t(s))$, with $t^{\prime}(s)+\left\|\partial_{s} u(s)\right\|_{L^{2}} \leq 1$ a.e., in the following sense:

$$
\begin{aligned}
& u_{n} \rightarrow u \text { weakly* in } L^{\infty}\left([0, S], H^{2}(\Omega)\right) \cap W^{1, \infty}\left([0, S], L^{2}(\Omega)\right), \\
& u_{n} \rightarrow u \text { strongly in } C\left([0, S], W^{2-\kappa, 2}(\Omega)\right), \quad \kappa>0, \\
& \partial_{s} u_{n} \rightarrow \partial_{s} u \text { weakly in } L^{1 / \kappa}\left([0, S], W^{\kappa, 2}(\Omega)\right), \quad 0 \leq \kappa<1, \\
& t_{n} \rightarrow t \text { weakly* in } W^{1, \infty}([0, S]), \\
& \int_{s_{2}}^{s_{1}} \widetilde{\Psi}\left(\partial_{s} u_{n}(s)\right) \mathrm{d} s \rightarrow \int_{s_{2}}^{s_{1}} \widetilde{\Psi}\left(\partial_{s} u(s)\right) \mathrm{d} s, \quad s_{1} \geq s_{2} \geq 0, \\
& \int_{s_{1}}^{s_{2}}\left(\partial \mathcal{C}_{\varepsilon_{n}}\left(\partial_{s} u_{n}(s), \partial_{s} u_{n}(s)\right)_{L^{2}} \mathrm{~d} s \rightarrow \int_{s_{1}}^{s_{2}}\left(\varphi_{0}(s), \partial_{s} u(s)\right)_{L^{2}} \mathrm{~d} s,\right.
\end{aligned}
$$

for a function $\varphi_{0} \in L^{2}\left([0, S], L^{2}(\Omega)\right)$ with $\varphi_{0}(s) \in \partial \mathcal{C}_{0}\left(\partial_{s} u(s)\right)$ for almost all $s$ 
The limit $(u, t)$ satisfies the following weakened version of (2.74):

$$
\left\{\begin{array}{l}
\partial \Psi\left(\partial_{s} u(s)\right)+\partial \mathcal{C}_{0}\left(\partial_{s} u(s)\right)+\mathcal{B} u(s)+f(t(s), u(s)) \ni 0 \\
t^{\prime}(s)+\left\|\partial_{s} u(s)\right\|_{L^{2}}=R(s) \\
u(0)=u_{0}, \quad t(0)=0
\end{array}\right.
$$

where the function $R \in L^{\infty}\left(\mathbb{R}_{+}\right)$satisfies $0 \leq R(s) \leq 1$ a.e. and the scaled time $t(s)$ satisfies

$$
s \geq t(s) \geq s / C-C, \quad 1 \geq t^{\prime}(s) \geq 0 .
$$

where the positive constant $C$ is independent of $s$.

The proof of this theorem is similar to the proof of Theorem 2.9 and based on the uniform estimates proved above and the convergence of subdifferentials established in Lemma A.4. The only difference is that we do not have now any strong convergence for $\partial_{s} u(s)$ and, for this reason, cannot prove that $R(s) \equiv 1$ by passing to the limit $\varepsilon \rightarrow 0$ in (2.68b). (As we will see in Section 2.6, $R(s)$ may be really strictly less than 1 in some examples.) Instead of this, we can only claim that $R(s) \leq 1$ using the weak convergence. The detailed proof of this theorem will be given in the next part for the more complicated quasi-linear case, see Theorem 4.16.

The next Corollary gives the straightforward lower bounds for the scaling factor $R(s)$ which will be improved in the next section using more refined methods.

Corollary 2.18. Let the assumptions of Theorem 2.17 hold. Then, for every $s_{2} \geq$ $s_{1} \geq 0$, the following estimate is valid:

$$
\int_{s_{2}}^{s_{1}} R(s) \mathrm{d} s \geq \min \left\{\left(s_{1}-s_{2}\right) / 2,\left(s_{1}-s_{2}\right)^{K} / C\right\}, \text { for } s_{1} \geq s_{2} \geq 0,
$$

where the positive constants $C$ and $K$ are independent of $s_{1}$ and $s_{2}$.

Proof. To prove (2.80), we will use the convergence (2.77e) and the interpolation estimate (2.54). For all intervals $I \subset[0, S]$ the latter combined with Hölder's inequality implies

$$
\|w\|_{L^{1}\left(I, L^{2}\right)} \leq C\|w\|_{L^{1}\left(I, L^{1}\right)}^{\gamma}\|w\|_{L^{1}\left(I, H^{1}\right)}^{1-\gamma} \leq C_{1}\|\Psi(w)\|_{L^{1}\left(I, L^{1}\right)}^{\gamma}\|w\|_{L^{1}\left(I, H^{1}\right)}^{1-\gamma},
$$

where $\gamma=\frac{2}{n+2}$ and $n$ is the space dimension. Integrating (2.68b) over $s \in I:=\left[s_{2}, s_{1}\right]$ and using (2.81) together with the bound (2.76) for $\partial_{s} u_{n}$ in $L^{1}\left(\left[s_{2}, s_{1}\right], H^{1}(\Omega)\right)$, we infer

$$
\begin{aligned}
t_{n}\left(s_{1}\right)-t_{n}\left(s_{2}\right) & =s_{1}-s_{2}-\int_{s_{2}}^{s_{1}}\left\|\partial_{s} u_{n}(s)\right\|_{L^{2}} \mathrm{~d} s \\
& \geq s_{1}-s_{2}-C\left(\int_{s_{2}}^{s_{1}}\left(\Psi\left(\partial_{s} u_{n}(s)\right), 1\right)_{L^{2}} \mathrm{~d} s\right)^{\gamma}
\end{aligned}
$$


where the constant $C$ is independent of $s_{1}, s_{2}$ and $n$. Finally, the convergence $(2.77 \mathrm{e})$ allows us to pass to the limit $n \rightarrow \infty$ in (2.82). Estimating the $L^{1}$ norm by the $L^{2}$ norm we obtain

$$
t\left(s_{1}\right)-t\left(s_{2}\right) \geq s_{1}-s_{2}-C_{2}\left(\int_{s_{2}}^{s_{1}}\left\|\partial_{s} u(s)\right\|_{L^{2}} \mathrm{~d} s\right)^{\gamma} .
$$

Using the second equation of (2.78) and $0 \leq v=\int_{s_{2}}^{s_{1}}\left\|\partial_{s} u\right\|_{L^{2}} \mathrm{~d} s \leq \int_{s_{2}}^{s_{1}} R(s) \mathrm{d} s$ we find

$$
\int_{s_{2}}^{s_{1}} R(s) \mathrm{d} s=v+t\left(s_{1}\right)-t\left(s_{2}\right) \geq s_{1}-s_{2}+v-C_{2} v^{\gamma} \geq s_{1}-s_{2}-C_{2}\left(\int_{s_{2}}^{s_{1}} R(s) \mathrm{d} s\right)^{\gamma} .
$$

Now it is easy to see that (2.80) holds with $K=1 / \gamma$ and $C=\left(2 C_{2}\right)^{K}$.

In the next section the lower bound (2.80) for $R$ is improved to $R(s) \geq \rho_{*}>0$ a.e. by more delicate arguments, i.e., we show that we may choose $K=1$ in (2.80).

Thus, due to Theorem 2.17 , the vanishing-viscosity solution $(u(s), t(s))$ satisfy the limit system (2.78) which differs from the intuitive limit equations (2.74) by the presence of a factor $R=R(s)$. However, systems (2.78) is, in a fact, not essentially different from (2.74) and can be transformed to it by a rescaling as described in the following remark.

Remark 2.19. We assume that $(t, u) \in W^{1, \infty}\left([0, S], \mathbb{R} \times L^{2}(\Omega)\right)$ satisfies $(2.78)$ and let

$$
\bar{s}(s):=\int_{0}^{s} R(s) \mathrm{d} s, \bar{S}:=\int_{0}^{S} R(s) \mathrm{d} s, \bar{u}(\bar{s}):=u(s(\bar{s})), \text { and } \bar{t}(\bar{s}):=t(s(\bar{s})) .
$$

Due to $(2.80)$, the function $\bar{s}:[0, S] \rightarrow[0, \bar{S}]$ is invertible, and the inverse function $s:[0, \bar{S}] \rightarrow[0, S]$ is Hölder continuous. Using the second equation of (2.78) and the regularity properties of $(u(s), t(s))$ obtained in Theorem 2.17 , one can easily verify that

$$
(\bar{t}, \bar{u}) \in W^{1, \infty}\left([0, \bar{S}], \mathbb{R} \times L^{2}(\Omega)\right) \text { and } \bar{t}^{\prime}(\bar{s})+\left\|\partial_{\bar{S}} \bar{u}(\bar{s})\right\|_{L^{2}}=1 \text { a.e. in }[0, \bar{S}] .
$$

Moreover, since $\partial_{s} u(s)=R(s) \partial_{\bar{s}} \bar{u}(\bar{s}(s))$ with $R(s) \in[0,1]$ we can use the special form of $\mathcal{C}_{0}$, which implies $\partial \mathcal{C}_{0}(\rho v) \subset \partial \mathcal{C}_{0}(v)$ for all $\rho \in[0,1]$ and $v$ with $\|v\|_{L^{2}}$ Thus, we obtain

$$
\partial \mathcal{C}_{0}\left(\partial_{s} u(s)\right) \subset \partial \mathcal{C}_{0}\left(\partial_{\bar{s}} \bar{u}(\bar{s}(s))\right) \quad \text { a.e. in }[0, S]
$$

Since the term $\partial \Psi\left(\partial_{s} u\right)$ is scaling invariant, then we can conclude that the scaled functions $(\bar{u}(\bar{s}), \bar{t}(\bar{s}))$ satisfies indeed (2.74). Therefore, the proved Theorem 2.17 gives, in particular, the existence of a solution of problem (2.74) belonging to the class (2.85). 
The next result provides some further regularity property. The proof will be given at the end of Section 2.5.

Corollary 2.20. Let the assumptions of Theorem 2.9 hold. Then, the solution $(\bar{u}(\bar{s}), \bar{t}(\bar{s}))$ of problem (2.74) constructed via the additional scaling (2.84) belongs to the following class

$$
\bar{u} \in W^{1, \infty}\left([0, S], L^{2}(\Omega)\right) \cap H^{1}\left([0, S], H^{1}(\Omega)\right) \cap L^{\infty}\left([0, S], H^{2}(\Omega)\right)
$$

for all $S>0$.

\subsection{Strict positivity of the scaling factor $R(s)$}

As we have seen at the end of the previous section, the convergence $(2.77 \mathrm{e})$ implies the Hölder continuity (2.80). In this section, we will show that both convergences (2.80)e) and (2.80)f) together with the higher energy estimate for problem (2.72) allow us to conclude that $K=1$ and, therefore the function $R(s)$ is separated from 0 almost everywhere. To be more precise, the following result holds.

Theorem 2.21. Let the assumptions of Theorem 2.9 hold and let $\left(u_{n}, t_{n}\right):=\left(u_{\varepsilon_{n}}, t_{\varepsilon_{n}}\right)$ be a sequence of solutions of (2.68) with $\varepsilon_{n} \rightarrow 0$ tending to the limit solution $(u, t)$ of problem (2.78) constructed in Theorem 2.17. Then, we additionally have

$$
\partial_{s} u_{n} \rightarrow \partial_{s} u \text { weakly in } L^{2}\left([0, S], H^{1}(\Omega)\right)
$$

and the limit function $R(s)$ in the second equation of (2.78) is strictly separated from 0 :

$$
1 \geq R(s) \geq \beta>0 \text { for almost all } s \in \mathbb{R},
$$

where the constant $\beta$ depends only on the $H^{2}$-norm of the initial data $u_{0}$.

The proof of this theorem is based on the following lemma which gives the analog of the higher energy estimate (2.30) for the solutions of the rescaled problem (2.68) (see also the proof of Theorem 4.17 below). In the proof we will use the functional

$$
\widehat{\mathcal{C}_{\varepsilon}}(v):=\left(\partial \mathcal{C}_{\varepsilon}(v), v\right)_{L^{2}}-\mathcal{C}_{\varepsilon}(v)=\varepsilon \hat{g}\left(\|v\|_{L^{2}}\right) \geq 0
$$

for a suitable function $\hat{g}:[0,1) \rightarrow[0, \infty)$. The construction of $\widehat{\mathcal{C}_{\varepsilon}}$ is such that

$$
\frac{d}{d s} \widehat{\mathcal{C}}_{\varepsilon}(v(s))=\left(D^{2} \mathcal{C}_{\varepsilon}(v(s)) v^{\prime}(s), v(s)\right)_{L^{2}},
$$

because of some cancellations.

Lemma 2.22. Let the above assumptions hold and let $(t(s), u(s))$ be a (unique strong) solution of problem (2.68). Then, for $0 \leq s_{1} \leq s_{2}$ we have, with $v(s)=$ $\partial_{s} u(s)$,

$$
\begin{aligned}
& \widehat{\mathcal{C}_{\varepsilon}}\left(v\left(s_{2}\right)\right)-\widehat{\mathcal{C}_{\varepsilon}}\left(v\left(s_{1}\right)\right)+\kappa \int_{s_{1}}^{s_{2}}\|v(s)\|_{H^{1}}^{2} \mathrm{~d} s \\
& \leq-\int_{s_{1}}^{s_{2}}\left(t^{\prime}(s) f_{t}^{\prime}(t(s), u(s))+f_{u}^{\prime}(t(s), u(s)) v(s), v(s)\right)_{L^{2}} \mathrm{~d} s .
\end{aligned}
$$


Estimate (2.90) can be verified exactly as we prove estimate (2.30) for solutions of problem (2.12) (by approximating the non-smooth term $\partial \Psi\left(\partial_{s} u\right)$ by $\Psi_{\delta}^{\prime}\left(\partial_{s} u\right)$, establishing this estimate for the auxiliary approximating problem (by differentiating the equation by $s$, multiplying it by $\partial_{s} u$, and employing (2.89)) and then passing to the limit $\delta \rightarrow 0$. So, we leave the proof of this lemma to the reader.

Proof of Theorem 2.21. We denote the right-hand side of (2.90) by $\mathcal{I}_{s_{1}, s_{2}}^{\varepsilon}$, when the solutions $\left(u_{\varepsilon}, t_{\varepsilon}\right)$ is inserted. Using (2.8) and (2.10) we find (analogously to (2.37))

$$
\begin{aligned}
\mathcal{I}_{s_{1}, s_{2}}^{\varepsilon} & \leq \int_{s_{1}}^{s_{2}}\left[t_{\varepsilon}^{\prime}(s)\left\|v_{\varepsilon}(s)\right\|_{H^{1-\gamma}}+\left\|v_{\varepsilon}(s)\right\|_{H^{1-\gamma}}^{2}\right] \mathrm{d} s \\
& \leq \int_{s_{1}}^{s_{2}}\left[\kappa / 2\left\|v_{\varepsilon}(s)\right\|_{H^{1}}^{2}+\mu t_{\varepsilon}^{\prime}(s)^{2}+C_{\mu}\left\|v_{\varepsilon}(s)\right\|_{L^{2}}^{2}\right] \mathrm{d} s,
\end{aligned}
$$

where $\mu>0$ is arbitrary and $C_{\mu}$ depends only on $\mu$ and the $H^{2}$-norm of $u_{0}$. Inserting this into (2.90) gives the estimate

$$
\widehat{\mathcal{C}_{\varepsilon}}\left(v\left(s_{2}\right)\right)-\widehat{\mathcal{C}_{\varepsilon}}\left(v_{\varepsilon}\left(s_{1}\right)\right)+\frac{\kappa}{2} \int_{s_{1}}^{s_{2}}\left\|v_{\varepsilon}(s)\right\|_{H^{1}}^{2} \mathrm{~d} s \leq \int_{s_{1}}^{s_{2}} \mu t^{\prime}(s)^{2}+C_{\mu}\left\|v_{\varepsilon}(s)\right\|_{L^{2}}^{2} \mathrm{~d} s .
$$

Using $t_{\varepsilon}^{\prime}(s)+\left\|\partial_{s} u_{\varepsilon}(s)\right\|_{L^{2}} \leq 1$ we deduce that

$$
\widehat{\mathcal{C}_{\varepsilon}}\left(v\left(s_{2}\right)\right)-\widehat{\mathcal{C}_{\varepsilon}}\left(v_{\varepsilon}\left(s_{1}\right)\right) \leq C_{1}\left(s_{2}-s_{1}\right)
$$

for $0 \leq s_{1}<s_{2} \leq S$ with $C_{1}$ independent of $\varepsilon$. Moreover, the explicit formula (2.70) for $\mathcal{C}_{\varepsilon}(v)$ and the a priori estimate (2.62) yield

$$
0 \leq \mathcal{C}_{\varepsilon}\left(v_{\varepsilon}(s)\right) \leq C \varepsilon \ln \frac{C}{\varepsilon} \quad \text { and } \quad 0 \leq \widehat{\mathcal{C}_{\varepsilon}}(v(s)) \leq C
$$

with $C$ depending only on the $H^{2}$-norm of $u_{0}$ (and is uniform with respect to $\varepsilon \rightarrow 0)$.

Thus, $s \mapsto \widehat{\mathcal{C}_{\varepsilon}}(v(s))-C_{1} s$ is nonincreasing and uniformly bounded. Using (2.91) for $s_{1}=0$ and $s_{2}=S$ and (2.93) provides uniform boundedness of $\partial_{s} u_{\varepsilon}=v_{\varepsilon}$ in $L^{2}\left([0, S], H^{1}(\Omega)\right)$. Thus, we are above to extract a subsequence $\varepsilon_{n} \rightarrow 0$ such that, for $\left(u_{n}, t_{n}\right)=\left(u_{\varepsilon_{n}}, t_{\varepsilon_{n}}\right)$ and $v_{n}=\partial_{s} u_{n},(2.87)$ holds and that

$$
\widehat{\mathcal{C}_{\varepsilon}}\left(v_{n}(s)\right) \rightarrow c_{0}(s) \text { for all } s \in[0, T] .
$$

Using $\mathcal{C}_{\varepsilon}\left(v_{\varepsilon}(s)\right) \rightarrow 0$ by (2.93), the definition of $\widehat{\mathcal{C}_{\varepsilon}}$, and the convergence (2.77f) we conclude

$c_{0}(s)=\left(\varphi_{0}(s), \partial_{s} v(s)\right)_{L^{2}}$ with $\varphi_{0} \in L^{2}\left([0, S], L^{2}(\Omega)\right)$ and $\varphi_{0}(s) \in \partial \mathcal{C}_{0}(v(s)) .(2.94)$ 
To verify estimate (2.88) we deduce some special integral estimate involving $R$ and $(u, t)$. For this we have to find a suitable estimate involving $\left(u_{n}, t_{n}\right)$ that allows us to pass to the limit. We use estimate (2.91) to infer

$$
\begin{aligned}
\int_{s_{1}}^{s_{1}}\left\|v_{n}(s)\right\|_{H^{1}}^{2} \mathrm{~d} s \leq & C\left(\widehat{\mathcal{C}}_{\varepsilon}\left(v_{n}\left(s_{1}\right)\right)-\widehat{\mathcal{C}}_{\varepsilon}\left(v_{n}\left(s_{2}\right)\right)\right)+\mu \int_{s_{1}}^{s_{2}} t^{\prime}(s)^{2} \mathrm{~d} s \\
& +C_{\mu} \int_{s_{1}}^{s_{2}}\left\|v_{n}(s)\right\|_{L^{1}(\Omega)}^{2} \mathrm{~d} s,
\end{aligned}
$$

where we have implicitly used the interpolation estimate in order to estimate the $L^{2}$ norm through $L^{1}$ and $H^{1}$ norms. Therefore, choosing $\mu=1 / 2$, using $\left\|v_{n}(s)\right\|_{L^{1}} \leq$ $C\left\|v_{n}(s)\right\|_{L^{2}}, t_{n}^{\prime}(s)+\left\|v_{n}(s)\right\|_{L^{2}} \leq 1$, and assumptions (2.3a), we get

$$
\begin{aligned}
s_{2}-s_{1} & =t_{n}\left(s_{2}\right)-t_{n}\left(s_{1}\right)+\int_{s_{1}}^{s_{2}}\left\|v_{n}(s)\right\|_{L^{2}} \mathrm{~d} s \\
& \leq t_{n}\left(s_{2}\right)-t_{n}\left(s_{1}\right)+\int_{s_{1}}^{s_{2}}\left\|v_{n}(s)\right\|_{H^{1}} \mathrm{~d} s \\
& \leq t_{n}\left(s_{2}\right)-t_{n}\left(s_{1}\right)+\frac{1}{2}\left(s_{2}-s_{1}\right)+\frac{1}{2} \int_{s_{1}}^{s_{2}}\left\|v_{n}(s)\right\|_{H^{1}}^{2} \mathrm{~d} s \\
& \leq \frac{1}{2}\left(s_{2}-s_{1}\right)+\frac{3}{2}\left(t_{n}\left(s_{2}\right)-t_{n}\left(s_{1}\right)\right)+C\left(\widehat{\mathcal{C}}_{\varepsilon}\left(v_{n}\left(s_{1}\right)\right)+\int_{s_{1}}^{s_{2}} \widetilde{\Psi}\left(v_{n}(s)\right) \mathrm{d} s\right),
\end{aligned}
$$

where $C$ depends on the $H^{2}$-norm of $u_{0}$, but is independent of $\varepsilon$. In this estimate we may pass to the limit $n \rightarrow \infty$, since all terms converge. After a simple rearrangement we find

$$
s_{2}-s_{1} \leq 3\left(t\left(s_{2}\right)-t\left(s_{1}\right)\right)+2 C c_{0}\left(s_{1}\right)+2 C \int_{s_{1}}^{s_{2}} \tilde{\Psi}(v(s)) \mathrm{d} s .
$$

This estimate is now used to obtain

$$
\begin{aligned}
\int_{s_{1}}^{s_{2}} R(s) \mathrm{d} s & =t\left(s_{2}\right)-t\left(s_{1}\right)+\int_{s_{1}}^{s_{2}}\|v(s)\|_{L^{2}} \mathrm{~d} s \\
& \geq t\left(s_{2}\right)-t\left(s_{1}\right)+\frac{1}{C_{2}} \int_{s_{1}}^{s_{2}} \widetilde{\Psi}(v(s)) \mathrm{d} s \\
& \geq \beta\left(3\left(t\left(s_{2}\right)-t\left(s_{1}\right)\right)+2 C \int_{s_{1}}^{s_{2}} \widetilde{\Psi}(v(s)) \mathrm{d} s\right) \\
& \geq \beta\left(s_{2}-s_{1}\right)-C_{2} c_{0}\left(s_{1}\right),
\end{aligned}
$$

with $\beta=1 / \max \left\{3,2 C_{1} C\right\}$.

To conclude $R(s) \geq \beta$ a.e., we consider the two alternative possibilities (i) $c_{0}\left(s_{1}\right)>0$ and (ii) $c_{0}\left(s_{1}\right)=0$. Since $s \mapsto c_{0}(s)-C_{1} s$ is nonincreasing, the 
function $c_{0}$ is continuous a.e. Thus, it suffices to consider $s_{1}$ in which $c_{0}$ is continuous. In case (i) the explicit description of $c_{0}$ in (2.94) gives $\left\|v\left(s_{1}\right)\right\|_{L^{2}}=1$ and, consequently, $R\left(s_{1}\right)=1 \geq \beta$. In case (ii) we also assume that $s_{1}$ is a Lebesgue point of $R$. Dividing (2.95) by $s_{2}-s_{1}$ and passing to the limit $s_{2} \rightarrow s_{1}$, we get $R\left(s_{1}\right) \geq \beta>0$. Thus, estimate (2.88) is verified and Theorem 2.21 is proved.

Remark 2.23. Differentiating equation (2.68) by $s$, multiplying it by $\partial_{s}^{2} u(s)$ and arguing as in the derivation of (2.31), one can obtain the uniform estimate of $\partial_{s} u_{\varepsilon}$ in the space $L^{\infty}\left(\left[s_{1}, S\right], H^{1}(\Omega)\right)$ for all $s_{1} \in(0, S)$. This shows that the limit solution $u$ of problem (2.78) satisfies $\partial_{s} u \in L_{\mathrm{loc}}^{\infty}\left((0, S], H^{1}(\Omega)\right)$.

Proof of Corollary 2.20. Indeed, due to estimate (2.88) both functions $\bar{s}(s)$ and $s(\bar{s})$ are uniformly Lipschitz continuous and, therefore, the regularity (2.86) of the function $\bar{u}$ follows immediately from the analogous regularity of $u(s)$ proved in Theorem 2.21.

\subsection{An example}

We conclude the section by showing that the result of Theorem 2.21 is, in a sense, sharp and cannot be, in general, improved till the desired equality $R(s) \equiv 1$. To this end, we first note that, although, for simplicity, we have considered above only the case where the non-linearity $f$ and the initial data $u_{0}$ are independent of $\varepsilon$, all of the results, obviously, remain true in the case where $f=f(\varepsilon, \cdot))$ and $u_{0}=u_{0}(\varepsilon)$ and the dependence on $\varepsilon$ is regular as $\varepsilon \rightarrow 0$.

Let us consider now the following system of two ODEs on the plane $u \in \mathbb{R}^{2}$ :

$$
\begin{cases}6 A \operatorname{sgn}\left(u_{1}^{\prime}\right)+\varepsilon u_{1}^{\prime}=\chi_{A}\left(u_{2}\right) u_{2}+f_{A}\left(u_{1}\right)+6 A+6 A^{2} \varepsilon-A t, & u_{1}(0)=0 \\ 6 A \operatorname{sgn}\left(u_{2}^{\prime}\right)+\varepsilon u_{u}^{\prime}=-\chi_{A}\left(u_{1}\right) u_{1}+f_{A}\left(u_{2}\right)+6 A+6 A^{2} \varepsilon+A t, & u_{2}(0)=\varepsilon\end{cases}
$$

where the nonlinearity $f_{A}(z)=0$ for $|z| \leq 2 A$ and is dissipative for large $z$. The parameter $A>1$ is fixed, and the cut-off function $\chi_{A}: \mathbb{R} \rightarrow[0,1]$ is such that $\chi_{A}(z) \equiv 1$ for $|z| \leq 2 A$ and 0 for $|z| \geq 3 A$.

On the one hand, we see that the assertion of the Theorem 2.21 still holds for this system. Indeed, the non-gradient part of the nonlinearity $f$ can be estimated via

$$
\left\|\left(\chi_{A}\left(u_{2}\right) u_{2},-\chi_{A}\left(u_{1}\right) u_{1}\right)\right\|_{\mathbb{R}^{2}} \leq 3 \sqrt{2} A
$$

and, consequently, the subordination condition (2.65) is satisfied, while the other assumptions are obvious. On the other hand, the unique solution $u_{\varepsilon}$ is given explicitly via

$$
u_{\varepsilon}(t)=\left(A t+\varepsilon \sin \frac{t}{\varepsilon}, A t+\varepsilon \cos \frac{t}{\varepsilon}\right) \text { for } t \in[0,1] .
$$

Obviously, for $\varepsilon \rightarrow 0$ this solution tends to the limit solution $u(t)=(A t, A t)$ of problem (2.96) with $\varepsilon=0$. Moreover, it follows from Theorem 2.21 that the scaled solutions $\left(t_{\varepsilon}(s), \tilde{u}_{\varepsilon}(s)\right)$ of problem (2.68) tend as $\varepsilon \rightarrow 0$ to a limit solution 
$\left(t_{0}(s), \tilde{u}_{0}(s)\right)$ of problem (2.78) with some $R(s)$ in the right-hand side of the second equation.

We show that $R(s)<1$ by an explicit calculation of the limit solution $\left(t_{0}, \tilde{u}_{0}\right)$. For $\varepsilon>0$ we have

$$
\begin{aligned}
s_{\varepsilon}(t) & =t+\int_{0}^{t} \sqrt{\left|u_{1, \varepsilon}^{\prime}(r)\right|^{2}+\left|u_{2, \varepsilon}^{\prime}(r)\right|^{2}} \mathrm{~d} r \\
& =t+\int_{0}^{t} \sqrt{\left(A+\cos \frac{r}{\varepsilon}\right)^{2}+\left(A-\sin \frac{r}{\varepsilon}\right)^{2}} \mathrm{~d} r
\end{aligned}
$$

and find the limit $s_{\varepsilon}(t) \rightarrow(1+B) t$ for $\varepsilon \rightarrow 0$ with

$$
B=\frac{1}{2 \pi} \int_{0}^{2 \pi} \sqrt{(A+\cos \phi)^{2}+(A-\sin \phi)^{2}} \mathrm{~d} \phi>\sqrt{2} A .
$$

Denote by $t_{\varepsilon}(s)$ the inverse of $s_{\varepsilon}(t)$, then with $\tilde{u}_{\varepsilon}(s)=u_{\varepsilon}\left(t_{\varepsilon}(s)\right)$ we have $t_{0}(s)=$ $\lim _{\varepsilon \rightarrow 0} t_{\varepsilon}(s)=\frac{s}{1+B}$ and $\tilde{u}_{0}(s)=\lim _{\mathcal{E} \rightarrow 0} \tilde{u}_{\varepsilon}(s)=\frac{A s}{1+B}(1,1)$. Thus, we find

$$
R(s)=t_{0}^{\prime}(s)+\left\|\tilde{u}_{0}^{\prime}(s)\right\|_{\mathbb{R}^{2}}=\frac{1}{1+B}+\frac{\sqrt{2} A}{1+B}=\frac{1+\sqrt{2} A}{1+B}<1 .
$$

\section{The abstract semilinear case: an alternative approach}

The aim of this section is to discuss briefly the general semilinear case

$$
0 \in \partial \tilde{\Psi}(\dot{u})+\varepsilon I \dot{u}+\mathcal{B} u+f(t, u), \quad u(0)=u_{0},
$$

where the underlying space is an abstract Hilbert space $H$ with scalar product $(\cdot, \cdot)$ and Riesz isomorhpism $I: H \rightarrow H^{*}$. We will identify $H$ and $H^{*}$ and hence drop the operator $I$. On the one hand, the analysis here is similar to the one given in the previous section, so we will just highlight the general principles without going into too much technicalities. On the other hand, we provide an alternative approach to obtain the central estimate for the control of $\int_{T}^{T+1}\|\dot{u}(t)\|_{H^{1}} \mathrm{~d} t$. In particular, we are able to do all the estimates in the original time variable $t$ and can avoid the usage of $\tau=\varepsilon t$ completely.

\subsection{Assumptions}

On the Hilbert space $H$ the linear operator $\mathcal{B}: D(\mathcal{B}) \subset H \rightarrow H$ is assumed to be self-adjoint with compact inverse and to be positive definite, i.e., there exists $\kappa>0$ with

$$
(\mathcal{B} u, u)_{H} \geq \kappa\|u\|^{2} \text { for all } u \in D(\mathcal{B}) .
$$

As usual, the operator $\mathcal{B}$ generates a scale of Hilbert spaces $H_{\alpha}:=D\left(\mathcal{B}^{\alpha / 2}\right), \alpha \in \mathbb{R}$, equipped with the (graph) norm $\|u\|_{\alpha}=\|u\|_{H_{\alpha}}=\left\|\mathcal{B}^{\alpha / 2} u\right\|=\left(\mathcal{B}^{\alpha} u, u\right)_{H}^{1 / 2}$ and $\|u\|_{0}=\|u\|$. The compactness of $\mathcal{B}^{-1}$ implies $H_{\alpha} \Subset H_{\beta}$ for $\beta<\alpha$. 
The dissipation functional $\widetilde{\Psi}: H \rightarrow[0, \infty)$ is assumed to be continuous, convex, homogeneous of degree 1 , and positive, i.e., for all $u, v \in H$ and $\alpha \geq 0$ we have

$$
\begin{array}{ll}
\widetilde{\Psi}(u) \leq C\|u\|, & \widetilde{\Psi}(u+v) \leq \widetilde{\Psi}(u)+\widetilde{\Psi}(v), \\
\widetilde{\Psi}(\alpha u)=\alpha \widetilde{\Psi}(u), & \widetilde{\Psi}(u)=0 \Leftrightarrow u=0 .
\end{array}
$$

From assumption (3.3a) we conclude that the subdifferential $\partial \Psi(u)$ is uniformly bounded in $H$ :

$$
\|\eta\| \leq C \text { for all } u \in H \text { and all } \eta \in \partial \Psi(u) .
$$

Usually, it is assumed that $\widetilde{\Psi}$ is coercive on a suitable Banach space $X$ into which the Hilbert space $H$ is continuously embedded. Here, we avoid the usage of the space $X$ and work instead with $\widetilde{\Psi}$ directly. The connection of $\widetilde{\Psi}$ with the Hilbert spaces $H_{\alpha}$ is incorporated into the interpolation condition

$$
\exists \theta \in(0,1), C>0: \quad\|u\| \leq C \tilde{\Psi}(u)^{\theta}\|u\|_{1}^{1-\theta} \text { for all } u \in H_{1} .
$$

The nonlinear operator $f(t, \cdot)$ is assumed to map $H_{1-\gamma}$ into $H_{-1+\gamma}$ for some $\gamma \in(0,1)$. Moreover, it is assumed to be potential, i.e., $f(t, u)=\Phi_{u}^{\prime}(t, u)$ for some nonlinear functional $\Phi(t, \cdot)$ on $H_{1-\gamma}$. In addition, we impose the following dissipativity assumptions analogous to $(2.7)$ :

$$
\begin{aligned}
& (f(t, u), u)_{H} \geq-C ; \\
& \Phi_{t}^{\prime}(t, u) \leq \kappa_{3}(f(t, u), u)_{H}+C\left(1+\|u\|^{2}\right) ; \\
& \Phi(t, u) \leq \kappa_{3}(f(t, u), u)_{H}+C\left(1+\|u\|^{2}\right) .
\end{aligned}
$$

Moreover, we impose the growth and regularity assumptions formulated as assertions in Lemma 2.1. This leads to the following assumptions for the abstract case:

$$
\begin{aligned}
& \|f(t, u)\|_{H_{-1+\gamma}}+\left\|f_{t}^{\prime}(t, u)\right\|_{H_{-1+\gamma}}+|\Phi(t, u)|+\left|\Phi_{t}^{\prime}(t, u)\right| \leq Q\left(\|u\|_{H_{1-\gamma}}\right) \text { and } \\
& \left|\left(f_{u}^{\prime}(t, u) v, v\right)_{H}\right| \leq Q\left(\|u\|_{H_{1-\gamma}}\right)\|v\|_{H_{1-\gamma}}^{2} \quad \text { for all } u, v \in H_{1-\gamma} \\
& \|f(t, u)\|_{H} \leq Q\left(\|u\|_{H^{1}}\right)\|u\|_{H_{2}}^{1-\gamma} \text { for all } u \in H_{2} ; \\
& \left\|f_{u}^{\prime}(t, u) v\right\|_{H} \leq Q\left(\|u\|_{H_{1}}\right)\left(1+\|u\|_{H_{2}}\right)\|v\|_{H_{1}} \text { for all } u \in H_{2}, v \in H_{1} \\
& \left\|f_{t}^{\prime}(t, u)\right\|_{H} \leq Q\left(\|u\|_{H_{2}}\right) \text { for all } u \in H_{2}
\end{aligned}
$$

for some positive $\gamma$ and a monotonously increasing function $Q$.

\subsection{Wellposedness and energy estimates}

Most parts of the estimates obtained in previous sections can be transferred word by word to the abstract semilinear case. For this reason, we only indicate them below 
leaving the proof to the reader. However, there is a difference here. Namely, the explicit construction of smooth approximations to $\Psi(v)$ given in Appendix A does not work in the infinite-dimensional case. Although it does not seem a big problem to construct smooth approximations for the infinite-dimensional case (using, e.g., the proper combination of Yosida approximations), we prefer to give below an alternative scheme which works directly with non-smooth potential $\Psi$ and Galerkin approximations.

As in Section 2.2 (see Definition 2.3), a function $u=u(t)$ is a weak energy solution of problem (3.1) if $u \in L^{\infty}\left([0, T], H_{1}\right) \cap H^{1}([0, T], H)$ and satisfies the equation as an equality in $L^{2}\left([0, T], H_{-1}\right)$. Moreover, exactly as in Lemma 2.4, we establish that every weak energy solution satisfies $u \in L^{2}\left([0, T], H_{2}\right)$ and $f(\cdot, u) \in L^{2}([0, T], H)$. In particular, this means that every weak solution satisfies the energy equality

$$
\mathcal{E}(t, u(t))+\int_{0}^{t} \tilde{\Psi}(\dot{u}(r))+\varepsilon\|\dot{u}(r)\|^{2} \mathrm{~d} r=\mathcal{E}(0, u(0))+\int_{0}^{t} \mathcal{E}_{t}^{\prime}(r, u(r)) \mathrm{d} r,
$$

where $\mathcal{E}(t, u)=\frac{1}{2}(\mathcal{B} u, u)+\Phi(t, u)$. In order to obtain this estimate, we need to multiply (3.1) by $\dot{u}$, use that $(\partial \Psi(\dot{u}), \dot{u})=\Psi(\dot{u})$ and integrate over $[0, t]$.

Furthermore, arguing exactly as in Theorem 2.8 (see the derivation of (2.34)), we have

$$
\|u(t)\|_{1}^{2}+\varepsilon \int_{t}^{t+1}\|\dot{u}(r)\|^{2} \mathrm{~d} r \leq Q\left(\|u(0)\|_{1}\right) \mathrm{e}^{-\alpha t / \varepsilon}+C_{*},
$$

where the positive constants $\alpha$ and $C_{*}$ and the monotone function $Q$ are independent of $\varepsilon$ and $u(0)$. This estimate, the energy equality (3.8) and assumption (3.6) gives the control of the dissipation via

$$
\int_{t_{1}}^{t_{2}} \widetilde{\Psi}(\dot{u}(t)) \mathrm{d} t \leq C\left(1+t_{2}-t_{1}\right) \text { for } 0 \leq t_{1} \leq t_{2},
$$

where the constant $C$ depends on $u(0)$, but is independent of $t_{1}, t_{2}$ and $\varepsilon$, see Corollary 2.10. Finally, we have the following wellposedness result for the weak energy solutions of problem (3.1).

Lemma 3.1. Let the above assumptions hold. Then, for every $u_{0} \in H_{1}$, problem (3.1) possesses a unique weak energy solution $u$ in the above described sense.

Indeed, the existence of a solution is standard (see e.g., [6]) and the uniqueness can be established exactly as in Proposition 2.7.

\subsection{Higher energy estimates}

As already mentioned, estimate (3.10) is not sufficient for passing to the vanishingviscosity limit $\varepsilon \rightarrow 0$. We need an analogous estimate for the $H$-norm (or $H_{1}$-norm) 
of $\dot{u}$. For this, we would like to differentiate the equation with respect to $t$ and write out the energy estimate for that differentiated equation. However, the term $\partial \Psi(\dot{u})$ is clearly not differentiable and, consequently, this procedure requires a non-trivial justification.

In the previous section, we managed this by constructing explicitly smooth approximations for the functional $\Psi$ and passing to the limit. In this section, we employ a technique using difference quotients $v_{h}(t)=\frac{1}{h}(u(t+h)-u(t))$, which was already successfully exploited in [18] for nonsmooth problems in plasticity. The idea is to derive estimates uniform in $h>0$ for $v_{h}$, which then implies that $\dot{u}$ exists and satisfies the same bounds.

We say that the energy solution $u$ of equation (3.1) is a strong solution if

$$
u \in L^{\infty}\left([0, T], H_{2}\right), \quad \dot{u} \in L^{2}\left([0, T], H_{1}\right) \cap L^{\infty}([0, T], H) .
$$

We first verify the smoothing property for strong solutions which is analogous to (2.31). That additional smoothness will be essentially used below for verifying the analog of the energy equality (2.22). In the subsequent Theorem 3.3 we then show that energy solutions are, in fact, strong solutions.

Lemma 3.2. Let the above assumptions hold. Then, any strong solution $u$ of problem (3.1) becomes more regular for $t>0$, namely

$$
\dot{u} \in L^{\infty}\left(\left[t_{1}, T\right], H_{1}\right) \cap H^{1}\left(\left[t_{1}, T\right], H\right) \quad \text { for all } t_{1} \in(0, T)
$$

and satisfies the following estimate:

$$
\varepsilon \int_{0}^{t} r\|\ddot{u}(r)\|^{2} \mathrm{~d} r+t\|\dot{u}(t)\|_{H_{1}}^{2} \leq \frac{C(t+1)}{\varepsilon} \int_{0}^{t}\|\dot{u}(r)\|_{H_{1}}^{2} \mathrm{~d} r .
$$

where the constant $C$ depends on the $L^{\infty}\left([0, T], H_{2}\right)$-norm of the solution $u$, but is independent of $t$ and $\varepsilon$.

Proof. Note that (3.1) is equivalent to

$$
\forall_{\text {a.a. }} t \in[0, T] \forall \hat{v} \in H: \quad(\varepsilon \dot{u}+\mathcal{B} u+f(t, u), \hat{v}-\dot{u})_{H}+\widetilde{\Psi}(\hat{v})-\tilde{\Psi}(\dot{u}) \geq 0 .
$$

Choosing $t$ and $h$ such that $t, t+h \in[0, T]$ and we may use (3.14) at $t$ and $t+h$ and test with $\hat{v}=\dot{u}(t+h)$ and $\hat{v}=\dot{u}(t)$, respectively. Adding the two estimates all terms involving $\widetilde{\Psi}$ cancel. Dividing by $h^{2}$ and using $v_{h}(t):=\frac{1}{h}(u(t+h)-u(t))$ we arrive at $\varepsilon\left\|\dot{v}_{h}(t)\right\|^{2}+\frac{1}{2} \frac{d}{d t}\left(\mathcal{B} v_{h}(t), v_{h}\right)_{H}+\left(\frac{1}{h}[f(t+h, u(t+h))-f(t, u(t))], \dot{v}_{h}(t)\right)_{H} \leq 0$.

Employing (3.7d) and (3.7e) gives

$$
\varepsilon\left\|\dot{v}_{h}(t)\right\|^{2}+\frac{d}{d t}\left(\mathcal{B} v_{h}(t), v_{h}(t)\right)_{H} \leq \frac{C}{\varepsilon}\left(1+\left\|v_{h}(t)\right\|_{1}^{2}\right),
$$


where the constant $C$ depends on the $L^{\infty}\left([0, T], H_{2}\right)$-norm of the solution $u$, but is independent of $t$ and $\varepsilon$. Multiplying this estimate by $t$ and integrating in time, we get

$$
\varepsilon \int_{0}^{t} r\left\|\dot{v}_{h}(r)\right\|^{2} \mathrm{~d} r+t\left\|v_{h}(t)\right\|_{1}^{2} \leq \frac{C(t+1)}{\varepsilon}\left(1+\int_{0}^{t}\left\|v_{h}(r)\right\|_{1}^{2} \mathrm{~d} r\right) .
$$

Since the strong solution $u$ is assumed belonging to $H^{1}\left([0, T], H_{1}\right)$, we may pass to the limit $h \rightarrow 0$ in estimate (3.15) which gives all the assertions of the lemma.

We now verify the analog of energy equality (2.22) for $\dot{u}$ that does not depend on $\Psi$. For this, we replace $v$ by $\lambda v$ in (3.14), use the 1-homogeneity in $v$ and divide by $\lambda>0$. Passing after that to the limit $\lambda \rightarrow \infty$, we have

$$
\forall_{\text {a.a. }} t \in[0, T] \forall v \in H: \quad(\varepsilon \dot{u}+\mathcal{B} u+f(t, u), v)_{H}+\Psi(v) \geq 0 .
$$

However, inserting $v=\dot{u}$ into here and $v=0$ into (3.14) we also obtain

$$
\forall_{\text {a.a. }} t \in[0, T]: \quad(\varepsilon \dot{u}+\mathcal{B} u+f(t, u), \dot{u})_{H}+\Psi(\dot{u})=0,
$$

which is just the differentiated version of the energy balance (3.8).

Let $g(t)=\varepsilon \dot{u}+\mathcal{B} u+f(t, u)$. Then, using the regularities (3.11) and (3.12), we conclude that $g \in A C\left((0, T), H_{-1}\right)$. Choosing now $v=\dot{u}\left(t_{*}\right)$ for some $t_{*} \in(0, T)$ (the element $v$ exists for all $t_{*}$ since $\dot{u} \in C((0, T], H)$ and even $C_{w}\left((0, T], H_{1}\right)$ due to the smoothing (3.12)), we have

$$
\left(g(t), \dot{u}\left(t_{*}\right)\right)_{H} \geq-\Psi\left(\dot{u}\left(t_{*}\right)\right)=\left(g\left(t_{*}\right), \dot{u}\left(t_{*}\right)\right)_{H}
$$

for all $t, t_{*} \in(0, T)$. Thus, the left-hand side as a function of $t$ attains its minimum at $t=t_{*}$. Since $g \in A C\left((0, T), H_{-1}\right)$, this implies that $g$ is differentiable a.e. with $\left(\dot{g}\left(t_{*}\right), \dot{u}\left(t_{*}\right)\right)_{H}=0$ for almost all $t_{*} \in(0, T)$. Inserting the definition of $g$, we establish the energy equality for the time derivative $\dot{u}$, namely

$$
\frac{\varepsilon}{2} \frac{\mathrm{d}}{\mathrm{d} t}\|\dot{u}\|^{2}+(\mathcal{B} \dot{u}, \dot{u})_{H}+\left(f_{u}^{\prime}(t, u) \dot{u}, \dot{u}\right)_{H}+\left(f_{t}^{\prime}(t, u), \dot{u}\right)_{H}=0 .
$$

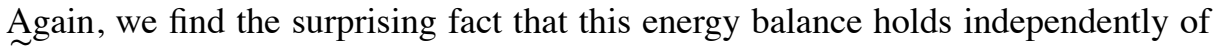
$\widetilde{\Psi}$.

We can now state the main result on the wellposedness for the abstract problem (3.1).

Theorem 3.3. Let the above assumptions hold and let $u_{0} \in H_{2}$. Then, the energy solution u of problem (3.1) is unique, is a strong solution, and satisfies the dissipative estimate

$$
\|u(t)\|_{H_{2}}^{2}+\varepsilon^{2}\|\dot{u}(t)\|^{2}+\varepsilon \int_{t}^{t+1}\|\dot{u}(s)\|_{H_{1}}^{2} \mathrm{~d} s \leq Q\left(\|u(0)\|_{H_{2}}\right) \mathrm{e}^{-\alpha t / \varepsilon}+C_{*}
$$

for some monotone function $Q$ and positive constants $\alpha$ and $C_{*}$, which are independent of $\varepsilon$, $u$ and $t \geq 0$. 
Proof. As in previous sections, the higher energy equality (3.17) together with the additional assumption $\dot{u} \in C([0, T], H)$ would allow us to establish the abstract analog of the dissipative estimate (3.18), which is the abstract analog of (2.29). Yet, we do not succeed to prove that any strong solution $u$ of problem (3.1) satisfies $u \in C^{1}([0, T], H)$, since Lemma 3.2 only implies $u \in C^{1}((0, T], H)$.

However, in the finite-dimensional case $\operatorname{dim} H<\infty$, this regularity is immediate and, in addition, any energy solution is automatically a strong solution. This observation allows us to overcome the above difficulty and establish the basic dissipative estimate (3.18) without the continuity assumption. To this end, we consider the standard Galerkin approximations to problem (3.1):

$$
0 \in \partial \tilde{\Psi}\left(\dot{u}_{N}\right)+\varepsilon \dot{u}_{N}+\mathcal{B} u_{N}+P_{N} f\left(t, u_{N}\right), \quad u_{N}(0)=P_{N} u_{0},
$$

where $P_{N}$ is an orthoprojector to the first $N$ eigenvalues of $\mathcal{B}$ and $u_{N} \in H_{N}:=$ $P_{N} H$. Since $u_{N} \in C^{1}\left([0, T], H_{N}\right)$ we can use (3.17) to derive the basic dissipative estimate (3.18) for $u_{N}$ with $Q$ and $C_{*}$ independent of $N$. Moreover, since $u_{0} \in H_{2}$ we also have $\left\|u_{N}(0)\right\|_{2} \leq\|u(0)\|_{2}=\left\|u_{0}\right\|_{2}$, such that the right-hand side will be uniformly bounded with respect to $N \rightarrow \infty$. Then passing to the limit $N \rightarrow \infty$, we establish the existence of a strong solution for problem (3.1) and the validity of the dissipative estimate (3.18). Thus, we have proved the theorem.

\subsection{Basic $L^{1}$ estimate for the time derivative}

We are now ready to verify the analog of estimate $(2.63)$, which provides the $L^{1}$ estimate with respect to $t \in[0, T]$ in the $H_{1}$ norm.

Lemma 3.4. Let the above assumptions hold and $u_{0} \in H_{2}$. Then, energy solutions $u$ of (3.1) satisfies the following estimates:

$$
\begin{aligned}
& \|\dot{u}(t)\| \leq C / \varepsilon \text { a.e.in }[0, T], \\
& \int_{t_{1}}^{t_{2}}\|\dot{u}(t)\|_{1} \mathrm{~d} t \leq C\left(1+t_{2}-t_{1}\right) \text { for } 0 \leq t_{1}<t_{2} \leq T,
\end{aligned}
$$

where the constant $C$ depends on $\left\|u_{0}\right\|_{2}$, but is independent of $\varepsilon, t_{1}, t_{2}$, and $T$.

Proof. Estimate (3.20) can be verified exactly as in the proof of Proposition 2.11. However, here we present an alternative, probably simpler proof, which works directly in the scaling of the time $t$ and thus avoids usage of the slow time $\tau=\varepsilon t$. Using the higher energy equality (3.17), together with (3.9) and the assumptions (3.7d) and (3.7e) we have

$$
\frac{\varepsilon}{2} \frac{d}{d t}\|v(t)\|^{2}+\kappa\|v(t)\|_{1}^{2} \leq C\left(1+\|v(t)\|_{1}\right)\|v(t)\|, \quad \text { where } v(t)=\dot{u}(t),
$$

for some positive constant $C$, which is independent of $t$ and $\varepsilon$. 
To simplify the notation in the subsequent arguments we introduce the shorthands

$$
\psi(t)=\widetilde{\Psi}(v(t)), \quad \mu(t)=\|v(t)\|, \quad \text { and } v(t)=\|v(t)\|_{1}
$$

for the different norms of the rate $v=\dot{u}$. Our assumptions give

$$
\psi \leq C \mu, \quad \mu \leq C \nu, \quad \mu \leq C \psi^{\theta} \nu^{1-\theta}, \quad \text { and } \mu(0) \leq C / \varepsilon .
$$

With these abbreviations (3.21) takes the form $\varepsilon \mu \dot{\mu}+\kappa v^{2} \leq C(1+v) \mu$. Using

$$
\mu \nu \leq C \psi^{\theta} v^{2-\theta} \leq \frac{\kappa}{2} v^{2}+C \psi^{2} \leq \frac{\kappa}{2} v^{2}+C^{2} \psi \mu
$$

we obtain the following the differential estimate $\varepsilon \mu \dot{\mu}+\frac{\kappa}{2 C} \mu \nu \leq C(1+\psi) \mu$. We first assume that $\mu>0$, then dividing by $\mu$ gives

$$
\dot{\mu}+\frac{\kappa}{2 \varepsilon C} v \leq \frac{C}{\varepsilon}(1+\psi) \text {. }
$$

Estimating $v \geq \mu / C$ we find $\dot{\mu}+\alpha_{\varepsilon} \mu \leq C(1+\psi) / \varepsilon$ with $\alpha_{\varepsilon}=\kappa /\left(2 \varepsilon C^{2}\right)$, which leads to

$$
\mu(t) \leq \mu(0) \mathrm{e}^{-\alpha_{\varepsilon} t}+\int_{0}^{t} \mathrm{e}^{-\alpha_{\varepsilon}(t-s)} \frac{C}{\varepsilon}(1+\psi(s)) \mathrm{d} s \quad \text { for } 0<t \leq T .
$$

Using $\mu(0) \leq C / \varepsilon$ and estimate (3.10) yields (3.20a). Integrating (3.22) we obtain

$$
\int_{t_{1}}^{t_{2}}\|\dot{u}(t)\|_{1} \mathrm{~d} t=\int_{t_{1}}^{t_{2}} v(t) \mathrm{d} t \leq \int_{t_{1}}^{t_{2}} \frac{2 C^{2}}{\kappa}(1+\psi(t)) \mathrm{d} t+\frac{2 \varepsilon C}{\kappa} \mu\left(t_{1}\right) .
$$

Together with (3.20a) we find the desired estimate (3.20b).

In case we do not have $\mu>0$, we may change the definition of $\psi, \mu$, and $v$ into

$$
\psi(t)=\left(\widetilde{\Psi}(v(t))^{2}+\delta\right)^{1 / 2}, \mu(t)=\left(\|v(t)\|^{2}+\delta\right)^{1 / 2} \text {, and } v(t)=\left(\|v(t)\|_{1}^{2}+\delta\right)^{1 / 2},
$$

where $\delta>0$ is arbitrary. It is easy to see that the above estimates remain valid with slightly larger constants. With $\delta \rightarrow 0$ the desired estimates are then established.

\subsection{The vanishing-viscosity limit}

Above we have derived uniform estimates with respect to $\varepsilon>0$. Thus, the the limit passage $\varepsilon \rightarrow 0$ in equation (3.1) can be done repeating the arguments given in sections 2.4 and 2.5 word by word. For this reason, we only formulate the main result and leave the details to the reader.

As in Section 2.4 we introduce the new scaled time

$$
s(t)=t+\int_{0}^{t}\|\dot{u}(t)\| \mathrm{d} t
$$


which is in fact the arc-length in the extended state space $[0, T] \times H$. Using the inverse $t_{\varepsilon}$ and $u_{\varepsilon}(s)=u\left(t_{\varepsilon}(s)\right.$ ) equation (3.1) takes the equivalent form (see also (2.68))

$$
\left\{\begin{array}{l}
0 \in \partial \widetilde{\Psi}\left(u_{\varepsilon}^{\prime}(s)\right)+\frac{\varepsilon}{1-\left\|u_{\varepsilon}^{\prime}(s)\right\|} u_{\varepsilon}^{\prime}(s)+\mathcal{B} u_{\varepsilon}(s)+f\left(t_{\varepsilon}(s), u_{\varepsilon}(s)\right) \\
t_{\varepsilon}^{\prime}(s)+\left\|u_{\varepsilon}^{\prime}(s)\right\|=1 \\
u_{\varepsilon}(0)=u_{0}, \quad t_{\varepsilon}(0)=0
\end{array}\right.
$$

where $^{\prime}=\frac{d}{d s}$.

Theorem 3.5. Let the assumptions of Theorem 3.3 hold and let $u_{\varepsilon}(0)=u_{0} \in \mathrm{H}_{2}$ be independent of $\varepsilon$. Then, there exists a sequence $\varepsilon_{n} \rightarrow 0$ such that the associated sequence $\left(t_{n}(s), u_{n}(s)\right):=\left(t_{\varepsilon_{n}}(s), u_{\varepsilon_{n}}(s)\right)$ of solutions of problems (3.23) converges to a limit pair $(t(s), u(s))$ in the sense of $(2.77 \mathrm{a}-\mathrm{f})$, where the spaces $W^{s, 2}(\Omega)$ are replaced by the spaces $H_{s}$. Moreover, the limit $(t(s), u(s))$ solves the analog of problem (2.78), namely

$$
\left\{\begin{array}{l}
0 \in \partial \widetilde{\Psi}\left(u^{\prime}(s)\right)+\partial \mathcal{C}_{0}\left(u^{\prime}(s)\right)+\mathcal{B} u(s)+f(t(s), u(s)) \\
t^{\prime}(s)+\left\|u^{\prime}(s)\right\|=R(s) \\
u(0)=u_{0}, \quad t(0)=0
\end{array}\right.
$$

where $\mathcal{C}_{0}(v)=0$ for $\|v\| \leq 1$ and $\infty$ else. Finally, the function $t(s)$ satisfies estimates (2.79) and the scaling factor $R(s)$ is such that $1 \geq R(s) \geq \beta>0$ for almost all $s$.

Of course, the solutions in the above result can be rescaled to in such a way that $R(s)=1$ a.e., see Remark 2.19 .

\subsection{BV solutions}

In [19-21] the concept of BV solutions is studied in oder to understand the pointwise limits of the unscaled function $u_{\varepsilon}(t)$. Such pointwise limits are sometimes also called approximable solutions, $c f$. $[13,19,27]$. However, it is desirable to characterize these solutions in terms of differential inclusions. We refer to [16] for a survey.

The name "BV solutions" derives from the fact that $\widehat{u}$ needs to lie in $B V([0, T], H)$. Thus for all $t \in[0, T]$ the limit $\widehat{u}\left(t^{+}\right)$and the limit $\widehat{u}\left(t^{-}\right)$from the left exist (in the strong $H$ topology). We can define the set $J(\widehat{u})$ of jump times, at which $\widehat{u}:[0, T] \rightarrow H$ is not continuous. A function $\widehat{u}:[0, T] \rightarrow H$ is called 
$B V$ solution of (3.1) if

$$
\left.\begin{array}{l}
\widehat{u} \in B V([0, T], H) \cap L^{\infty}\left([0, T], H_{1}\right) ; \\
0 \in \partial \Psi(0)+\mathcal{B} \widehat{u}(t)+f(t, \widehat{u}(t)) \quad \text { for all } t \in[0, T] \backslash J(\widehat{u}) ; \\
\forall t \in J(\widehat{u}) \exists \lambda_{t}>0 \exists y_{t} \in W^{1, \infty}([0,1], H) \cap L^{\infty}\left([0, T], H_{1}\right): \\
\widehat{u}\left(t^{-}\right)=y_{t}(0), \widehat{u}\left(t^{+}\right)=y_{t}(1), \widehat{u}(t)=y_{t}\left(\theta_{t}\right) \text { for some } \theta^{t} \in[0,1], \\
0 \in \partial \Psi\left(y_{t}^{\prime}(\theta)\right)+\partial \mathcal{C}_{0}\left(\lambda_{t} y_{t}^{\prime}(\theta)\right)+\mathcal{B} y_{t}(\theta)+f\left(t, y_{t}(\theta)\right) \text { for a.a. } \theta \in[0,1] ;
\end{array}\right\}
$$

where $\mathcal{E}(t, u)=\frac{1}{2}\langle\mathcal{B} u, u\rangle+\Phi(t, u)$ and $\Delta:[0, T] \backslash J(\widehat{u}) \rightarrow \mathbb{R}$ is defined via

$$
\Delta(t)=\int_{0}^{t} \widetilde{\Psi}(\mathrm{d} \widehat{u})+\sum_{\tau \in[0, t] \cap J(\widehat{u})}\left(\mathbb{J}(\tau)-\widetilde{\Psi}\left(\widehat{u}\left(\tau^{+}\right)-\widehat{u}\left(\tau^{-}\right)\right)\right)
$$

with $\mathbb{J}(\tau)=\int_{0}^{1} \widetilde{\Psi}\left(y_{\tau}^{\prime}(\theta)\right)+\left\|y_{\tau}^{\prime}(\theta)\right\| M\left(\mathcal{B} y_{\tau}(\theta)+f\left(\tau, y_{\tau}(\theta)\right)\right) \mathrm{d} \theta$ and $M(\xi)=$ $\min \{\|\xi+\kappa\|: \kappa \in \partial \widetilde{\Psi}(0)\}$.

The last condition (3.25d) is the energy balance corresponding to obtained from the viscous energy balance (3.8). The additional terms in $\Delta$ arising at the jumps are obtained as limit contributions of $\int_{0}^{T} \varepsilon\left\|\dot{u}_{\varepsilon}(s)\right\|^{2} \mathrm{~d} s$. Because (3.25c) postulates the existence of optimal jump paths, this notion is called connectable BV solution in [16, Def.4.21].

Clearly, the parametrized solutions constructed in Theorem 3.5 provide such BV solutions in the following way. For a parametrized solution $(t, u):[0, S] \rightarrow$ $\mathbb{R} \times H_{1}$ we let $T=t(S)$ and choose for each $t \in[0, T]$ an $s=\sigma(t) \in[0, S]$ such that $t(\sigma(t))=t$. Since the function $t:[0, S] \rightarrow[0, T]$ is nondecreasing and Lipschitz continuous, we see that every such $\sigma:[0, T] \rightarrow[0, S]$ is strictly increasing. We define $\widehat{u}(t)=u(\sigma(t))$ and claim that $\widehat{u}$ is a BV solution.

We sketch the arguments and refer to [16] for the details. First note that

$$
\begin{aligned}
\operatorname{Var}_{H}(\widehat{u},[0, T]) & :=\sup \left\{\sum_{i=1}^{N}\left\|\widehat{u}\left(t_{j}\right)-\widehat{u}\left(t_{j-1}\right)\right\|_{H}, N \in \mathbb{N}, 0 \leq t_{0}<t_{1}<\cdots<t_{N} \leq T\right\} \\
& \leq \operatorname{Var}_{H}(u,[0, S]) \leq \int_{0}^{S} R(s) \mathrm{d} s<\infty .
\end{aligned}
$$

which provides $\widehat{u} \in B V([0, T], H)$. Moreover, $u \in L^{\infty}\left([0, S], H_{1}\right)$ implies $\widehat{u} \in$ $L^{\infty}\left([0, T], H_{1}\right)$ and (3.25a) is established. The local stability condition (3.25b) follows from the first equation in (3.24). If $t_{*} \notin J(\widehat{u})$, then there is a unique $s_{*}$ with $t_{*}=t(s)$ and there is a sequence of Lebesgue points $s_{k} \rightarrow s_{*}$ of $u^{\prime}$ such that $\left\|u^{\prime}\left(s_{k}\right)\right\|<1$. Thus, the first equation in (3.24) holds with $\partial \widetilde{\Psi}\left(u^{\prime}\left(s_{k}\right)\right)+\partial \mathcal{C}_{0}\left(u^{\prime}\left(s_{k}\right)\right)$ 
replaced by $\partial \widetilde{\Psi}(0)$, since rate independence gives $\partial \widetilde{\Psi}(v) \subset \partial \widetilde{\Psi}(0)$. We conclude that (3.25b) holds at $t_{k}=t\left(s_{k}\right) \rightarrow t_{*}$. Because of $t \notin J(\widehat{u})$ we know also that $t \mapsto \widehat{u}(t) \in H_{1}$ is weakly continuous in $H_{1}$. Since $\partial \widetilde{\Psi}(0) \subset H_{1}^{*}$ is weakly closed we conclude that (3.25b) also holds at $t_{*}$.

For $t_{*} \in J(\widehat{u})$ we use the fact, that the preimage of $s \mapsto t(s)$ of $t_{*}$ is a full interval $\left[s_{1}^{*}, s_{2}^{*}\right]$. The desired jump path $y_{t_{*}}$ is then given via $y_{t_{*}}(\theta)=u\left(s_{1}^{*}+\theta\left(s_{2}^{*}-s_{1}^{*}\right)\right)$. The energy balance (3.25d) can be obtained by integrating along the parametrized curved and then identifying the jump terms accordingly.

As a consequence of this fact, it is possible to show that from each family $u_{\varepsilon}:[0, T] \rightarrow H_{1}$ of solutions of (3.1) with $u_{0} \in H_{2}$, we can extract a subsequence $u_{m}=u_{\varepsilon_{m}}$ with $\varepsilon_{m} \rightarrow 0$, such that for all $t \in[0, T]$ we have $u_{m}(t) \rightarrow \widehat{u}(t)$ weakly in $H$, where $\widehat{u}:[0, T] \rightarrow H_{1}$ is a BV solution. We refer to [20, Thm.4.10] or [16, Thm.4.23] for the details.

\section{The quasi-linear case}

In that chapter, we consider the general quasi-linear case with convex leading part. In addition, we do not assume that the underlying domain $\Omega$ is smooth, but only Lipschitz continuous. The main difficulty here, in comparison with the semi-linear case studied before, is the absence of the uniqueness result (even for the case of strong solutions). Moreover, we cannot even verify that all reasonably defined solutions satisfy the higher energy estimate (which is crucial for the rate-independent limit). By this reason, we have to proceed in much more delicate way including, in particular, the required higher energy estimate into the definition of a solution.

As in the previous chapter, the rigorous formulation of the problem, assumptions on $B, \Psi$ and $F$ and some basic preliminary facts are given in Section 4.1. The uniform with respect to $\varepsilon \rightarrow 0$ estimates and the existence of a solution for the general quasi-linear case is proved in Section 4.2 and the passage to the vanishingviscosity limit $\varepsilon \rightarrow 0$ is justified in Section 4.3.

\subsection{Preliminaries}

In this section we start to study the quasi-linear problem of the form:

$$
\left\{\begin{array}{l}
\varepsilon \partial_{t} u+\partial \Psi\left(\partial_{t} u\right) \ni \operatorname{div} b\left(\nabla_{x} u\right)-f(t, u), \\
\left.u\right|_{\partial \Omega}=0, \quad u(0, \cdot)=u_{0}
\end{array}\right.
$$

where $u(t, x)=\left(u^{1}(t, x), \cdots, u^{m}(t, x)\right)$ is an unknown vector-valued function, $\Omega \Subset \mathbb{R}^{n}$ is a bounded domain in $\mathbb{R}^{n}$ which is now only Lipschitz continuous and $\varepsilon>0$ is a small positive parameter.

We assume that the nonlinear function $b: \mathbb{R}^{m n} \rightarrow \mathbb{R}^{m n}$ has a gradient structure

$$
b(w)=\nabla_{w} B(w), \quad w \in \mathbb{R}^{n m}, \text { for some } B \in C^{2}\left(\mathbb{R}^{n m}, \mathbb{R}\right)
$$


and satisfies the following assumptions:

$$
\left\{\begin{array}{l}
\text { 1. }(b(w), w)_{\mathbb{R}^{n m}} \geq \alpha\|w\|^{p}-C, \\
\text { 2. }\left\|b^{\prime}(w)\right\| \mathcal{L}\left(\mathbb{R}^{n m}, \mathbb{R}^{n m}\right) \leq C\left(1+\|w\|^{p-2}\right), \\
\text { 3. }\left(b^{\prime}(w) \theta, \theta\right)_{\mathbb{R}^{n m}} \geq \kappa\|\theta\|_{\mathbb{R}^{n m}}^{2}, \quad \kappa>0
\end{array}\right.
$$

for some $p \geq 2$. In particular, first and second assumptions of (4.3) imply that

$$
\alpha_{1}\|w\|^{p}-C_{1} \leq B(w) \leq \alpha_{2}\|w\|^{p}+C_{2}
$$

for some positive $C_{i}$ and $\alpha_{i}$ and the third assumption of (4.3) guarantees that the potential $B(w)$ is strictly convex. Thus, the associated differential operator

$$
\mathcal{B}(u):=-\operatorname{div} b\left(\nabla_{x} u\right)
$$

is a non-degenerate second order quasilinear elliptic operator. Moreover, the above assumptions imply also that the operator $\mathcal{B}$ is a potential maximal monotone operator in $W_{0}^{1, p}(\Omega)$ (with respect to the standard inner product of $L^{2}(\Omega)$ ).

As in the semilinear case, we assume that $\partial \Psi(v)$ is a subdifferential of of a convex order one homogeneous functional $\Psi$ on $\mathbb{R}^{m}$, i.e., the function $\Psi: \mathbb{R}^{m} \rightarrow \mathbb{R}$ satisfies assumptions (2.3).

Finally the third nonlinearity $f: \mathbb{R} \times \mathbb{R}^{m} \rightarrow \mathbb{R}^{m}$ is supposed to be potential (i.e. (2.4) is assumed to be true) and to satisfy the growth restrictions (2.5) where the limit exponent exponent is now defined by $r<r_{\max }:=\frac{2 p}{n-p}\left(q_{\max }=\infty\right.$ if $p \geq n$ ). Moreover, the dissipativity assumption (2.6) is also takes place.

In particular, exactly as in Section 2.1, growth restrictions (2.5) and the dissipativity assumption (2.6) imply that

$$
F_{t}^{\prime}(t, u) \leq \kappa_{3} f(t, u) \cdot u+C\left(1+|u|^{r}\right), \quad \kappa_{3}>0 .
$$

Analogously to Lemma 2.1, the following lemma shows, the growth restrictions (2.5) guarantee that the term $f(t, u)$ is subordinated to the main elliptic operator $\mathcal{B}(u)$ and collects all necessary properties of the non-linear function $f(t, u)$.

Lemma 4.1. Let the function $f$ satisfy growth restrictions (2.5). Then,

1) The operators $u \rightarrow f(t, u)$ and $u \rightarrow f_{t}^{\prime}(t, u)$ are uniformly bounded continuous operators from $W^{1-\gamma, p}(\Omega)$ to $H^{-1}(\Omega)$, for some $\gamma>0$

$$
\|f(t, u)\|_{H^{-1}(\Omega)}+\left\|f_{t}^{\prime}(t, u)\right\|_{H^{-1}(\Omega)} \leq C\left(1+\|u\|_{W^{1-\gamma, p}(\Omega)}^{r+1}\right)
$$

with $C$ independent of $u$ and $t$.

2) The functionals $u \rightarrow(F(t, u), 1)_{L^{2}}$ and $u \rightarrow\left(F_{t}^{\prime}(t, u), 1\right)_{L^{2}}$ are bounded and continuous on $W^{1-\gamma, p}(\Omega)$ :

$$
\left|(F(t, u), 1)_{L^{2}}\right|+\left|\left(F_{t}^{\prime}(t, u), 1\right)_{L^{2}}\right| \leq C\left(1+\|u\|_{W^{1-\gamma, p}(\Omega)}^{r+2}\right) .
$$


Here and below $(u, v)_{L^{2}}$ denotes the scalar product in $L^{2}(\Omega)$.

3) The functional $u, v \rightarrow\left(f_{u}^{\prime}(t, u) v, v\right)_{L^{2}}$ is bounded and continuous on $W^{1-\gamma, p}(\Omega) \times W^{1-\gamma, 2}(\Omega)$ and

$$
\left|\left(f_{u}^{\prime}(t, u) v, v\right)_{L^{2}}\right| \leq C\left(1+\|u\|_{W^{1-\gamma, p}(\Omega)}^{r}\right)\|v\|_{W^{1-\gamma, 2}(\Omega)}^{2},
$$

where the constant $C$ is independent of $t, u$ and $v$.

Proof. Since the assertions of the lemma are more or less standard, we check below only the most complicated estimate (4.9) (which defines the limit exponent $r_{\max }$ ) leaving all other assertions to the reader. Indeed, due to (2.5),

$$
\left|\left(f_{u}^{\prime}(t, u) v, v\right)_{L^{2}}\right| \leq C\left(\|v\|_{L^{2}}^{2}+\left(|u|^{r}, v^{2}\right)_{L^{2}}\right) .
$$

Thus, we only need to estimate the second term in the right-hand side of (4.10). To this end, we will use the Sobolev embeddings $W^{1-\gamma, p} \subset L^{s_{\gamma}}$ and $W^{1-\gamma, 2} \subset L^{r_{\gamma}}$ with the exponents $r_{\gamma}$ and $s_{\gamma}$ satisfying

$$
\frac{1}{r_{\gamma}}=\frac{1}{2}-\frac{1-\gamma}{n}, \frac{1}{s_{\gamma}}=\frac{1}{p}-\frac{1-\gamma}{n} .
$$

Then, by the Hölder inequality, we want to have

$$
\left(|u|^{r}, v^{2}\right)_{L^{2}} \leq C\|u\|_{L^{s \gamma}(\Omega)}^{r}\|v\|_{L^{r_{\gamma}(\Omega)}}^{2} \leq C_{1}\|u\|_{W^{1-\gamma, p}(\Omega)}^{r}\|v\|_{W^{1-\gamma, 2}(\Omega)}^{2} .
$$

In order this estimate to be true, we need

$$
\frac{r}{s_{\gamma}}+\frac{2}{r_{\gamma}} \leq 1 \quad \text { or, equivalently, } \frac{r}{r_{\max }}+\gamma \frac{r+2}{2 n} \leq 1
$$

with $r_{\max }=\frac{2 p}{n-p}$. We see that condition (4.12) is satisfied for sufficiently small $\gamma>$ 0 if $r<r_{\max }$. Since estimate (4.11) implies (4.9), then Lemma 4.1 is proved.

Remark 4.2. We note that the theory developed below remains true if we add timeindependent monotone function $f_{0}(u)$ (i.e., with $f_{0}^{\prime}(u) \geq 0$ ) of arbitrary growth to the non-linearity $f$.

As in the semilinear case, in order to study the dependence of solutions of problem (4.1), it is more convenient to scale time $\tau=\varepsilon t$ and rewrite the problem in the equivalent form with respect to the function $\tilde{u}(\tau):=u(\varepsilon \tau)$. In order to simplify the notations, we will write below $u(\tau)$ instead of $\tilde{u}(\tau)$. Then, using that $\Psi$ is homogeneous of order one (and, consequently, $\partial \Psi$ is homogeneous of order zero), equation (4.1) reads

$$
\left\{\begin{array}{l}
\partial_{\tau} u+\partial \Psi\left(\partial_{\tau} u\right) \ni \operatorname{div} b\left(\nabla_{x} u\right)-f_{\varepsilon}(\tau, u), \\
\left.u\right|_{\partial \Omega}=0,\left.\quad u\right|_{\tau=0}=u_{0}
\end{array}\right.
$$


where, by definition,

$$
f_{\varepsilon}(\tau, u):=f(\varepsilon \tau, u) .
$$

In order to define the (strong) solution of equation (4.13), we first introduce a function

$$
\Theta_{u}(\tau):=\operatorname{div} b\left(\nabla_{x} u(\tau)\right)-f_{\varepsilon}(\tau, u(\tau)) .
$$

Definition 4.3. Let the above assumptions hold and let $u_{0} \in W_{0}^{1, p}(\Omega)$ is such that

$$
\Theta_{u}(0):=\operatorname{div} b\left(\nabla_{x} u_{0}\right)-f_{\varepsilon}\left(0, u_{0}\right) \in L^{2}(\Omega) .
$$

We say that a function $u=u(\tau, x)$ is a solution of (4.13) if, for every $R>0$,

$$
\left\{\begin{array}{l}
\text { 1) } u \in L^{\infty}\left([0, R], W_{0}^{1, p}(\Omega)\right), \\
\text { 2) } \partial_{\tau} u \in L^{\infty}\left([0, R], L^{2}(\Omega)\right) \cap L^{2}\left([0, R], H^{1}(\Omega)\right), \\
\text { 3) } \Theta_{u} \in L^{\infty}\left([0, R], L^{2}(\Omega)\right),
\end{array}\right.
$$

$u$ satisfies equation (4.26) in the sense of distributions and the following estimate

$$
\begin{aligned}
& \left\|\partial_{\tau} u\left(\tau_{2}\right)\right\|_{L^{2}}^{2}-\left\|\partial_{\tau} u\left(\tau_{1}\right)\right\|_{L^{2}}^{2}+2 \alpha \int_{\tau_{1}}^{\tau_{2}}\left\|\nabla_{x} \partial_{\tau} u(\tau)\right\|_{L^{2}}^{2} \mathrm{~d} \tau \\
& +2 \int_{\tau_{1}}^{\tau_{2}}\left[\left(f_{\varepsilon, \tau}^{\prime}(\tau, u(\tau)), \partial_{\tau} u(\tau)\right)_{L^{2}}+\left(f_{\varepsilon, u}^{\prime}(\tau, u(\tau)) \partial_{\tau} u(\tau), \partial_{\tau} u(\tau)\right)_{L^{2}}\right] \mathrm{d} \tau \leq 0
\end{aligned}
$$

holds for almost all $\tau_{2} \geq \tau_{1} \geq 0$ including $\tau_{1}=0$ (i.e., (4.18) holds for all $\tau_{1}, \tau_{2} \in$ $\mathbb{R}_{+} \backslash N, \tau_{2} \geq \tau_{1}$, with meas $N=0$ and $\left.0 \notin N\right)$. Here $\alpha>0$ is the same as in estimate (4.3)(1).

We now comment the requirements involved into the above solution's definition. We start with the third assumption of (4.17) on the $L^{2}$-regularity of the right-hand side $\Theta_{u}$ of equation (4.13). Let us consider the particular case $p=2$ and assume that the quasilinear elliptic operator $\mathcal{B}(u)$ possesses the $L^{2}$-maximal regularity property of the form: if

$$
\mathcal{B}(u):=-\operatorname{div} b\left(\nabla_{x} u\right)=h
$$

for some $u \in H_{0}^{1}(\Omega)$ and $h \in L^{2}(\Omega)$ then, necessarily, $u \in H^{2}(\Omega)$ and

$$
\|u\|_{H^{2}(\Omega)} \leq C\|h\|_{L^{2}}
$$

for some positive constant $C$ which can depend, in general on the $H^{1}$-norm of $u$.

Then, arguing as in Lemma 2.4, one can easily show that

$$
u_{0} \in H^{2}(\Omega) \text { and } u \in L^{\infty}\left(\mathbb{R}_{+}, H^{2}(\Omega)\right)
$$

in a complete agreement with the definition of a strong solution for the semilinear case, see Definition 2.6. 
However, we do not know the analog of such simple description for $p \neq 2$. Moreover, even for $p=2$, the maximal $\left(L^{2}, H^{2}\right)$-regularity for the elliptic operator $\mathcal{B}(u)$ is rather essential restriction and can be violated even in the linear case ( $\mathcal{B}$ is a linear operator) if the underlying domain is not $C^{1}$, see $e . g$. [24].

In contrast to that, as we will see below, assumption (4.17)(3) defines a reasonable class of solutions even in the general quasilinear situation described above.

Let us now consider the integral estimate (4.18). In a fact, it is nothing more than the integrated version of the higher order energy equality (2.22) (where the term $\left(\mathcal{B}^{\prime}(u) \partial_{t} u, \partial_{t} u\right)$ is replaced by its natural estimate from (4.3)(3)). However, in contrast to the semilinear case, we cannot verify that the strong solution satisfies automatically the higher energy equality (2.22) (or some reasonable estimate of that form) and have to include it into the definition of a strong solution. Thus, we have excluded from consideration the possible pathological solutions which do not satisfy the higher energy estimate (remind that the solution of problem (4.1) may be not unique and a pathological solution may exist simultaneously with a reasonable solution(s) for the same initial data).

It also worth to note that, in contrast to the semilinear case, we do not know whether or not the function $\tau \rightarrow\left\|\partial_{\tau} u(\tau)\right\|_{L^{2}}$ is continuous and, by this reason, we cannot obtain (4.18) for all $\tau_{1}, \tau_{2}$, but for almost all only.

We now verify that any strong solution satisfies the usual energy equality. To this end, we need the following lemma which is of independent interest as well.

Lemma 4.4. Let the function $b$ satisfy (4.3) and let

$$
\begin{aligned}
& \partial_{\tau} u \in L^{2}\left([0, R], H_{0}^{1}(\Omega)\right), \\
& u \in L^{\infty}\left([0, R], W_{0}^{1, p}(\Omega)\right), \\
& \operatorname{div} b\left(\nabla_{x} u\right) \in L^{2}\left([0, R], H^{-1}(\Omega)\right) .
\end{aligned}
$$

Then, $\left(B\left(\nabla_{x} u\right), 1\right)_{L^{2}} \in A C([0, R])$ and

$$
\frac{d}{d \tau}\left(B\left(\nabla_{x} u(\tau)\right), 1\right)_{L^{2}}=-\left(\operatorname{div} b\left(\nabla_{x} u(\tau)\right), \partial_{\tau} u(\tau)\right)_{L^{2}}
$$

for almost all $\tau$.

Proof. Let $h>0$ be arbitrary. Then, since $b^{\prime} \geq 0$, we have the following estimates:

$$
\begin{aligned}
& \frac{B\left(\nabla_{x} u(\tau+h)\right)-B\left(\nabla_{x} u(\tau)\right)}{h}=b\left(\nabla_{x} u(\tau)\right) \frac{\nabla_{x} u(\tau+h)-\nabla_{x} u(\tau)}{h} \\
& +\int_{0}^{1} r_{2} b^{\prime}\left(\nabla_{x} u(\tau)+r_{1} r_{2}\left[\nabla_{x} u(\tau+h)-\nabla_{x} u(\tau)\right]\right) \mathrm{d} r_{1} \mathrm{~d} r_{2} \frac{\left(\nabla_{x} u(\tau+h)-\nabla_{x} u(\tau)\right)^{2}}{h} \\
& \geq b\left(\nabla_{x} u(\tau)\right) \frac{\nabla_{x} u(\tau+h)-\nabla_{x} u(\tau)}{h}
\end{aligned}
$$


and

$$
\begin{aligned}
& \frac{B\left(\nabla_{x} u(\tau+h)\right)-B\left(\nabla_{x} u(\tau)\right)}{h}=b\left(\nabla_{x} u(\tau+h)\right) \frac{\nabla_{x} u(\tau+h)-\nabla_{x} u(\tau)}{h} \\
& -\int_{0}^{1}\left(1-r_{2}\right) b^{\prime}\left(\nabla_{x} u(\tau+h)-r_{1}\left(1-r_{2}\right)\left[\nabla_{x} u(\tau+h)-\nabla_{x} u(\tau)\right]\right) \mathrm{d} r_{1} \mathrm{~d} r_{2} \\
& \quad \times \frac{\left(\nabla_{x} u(\tau+h)-\nabla_{x} u(\tau)\right)^{2}}{h} \\
& \leq b\left(\nabla_{x} u(\tau+h)\right) \frac{\nabla_{x} u(\tau+h)-\nabla_{x} u(\tau)}{h} .
\end{aligned}
$$

Integrating these estimates with respect to $(t, x)$, we have

$$
\begin{aligned}
& -\int_{\tau_{1}}^{\tau_{2}}\left(\operatorname{div} b\left(\nabla_{x} u(\tau)\right), \frac{u(\tau+h)-u(\tau)}{h}\right)_{L^{2}} \mathrm{~d} \tau \\
& \leq \frac{1}{h} \int_{\tau_{2}}^{\tau_{2}+h}\left(B\left(\nabla_{x} u(\tau)\right), 1\right)_{L^{2}} \mathrm{~d} \tau-\frac{1}{h} \int_{\tau_{1}}^{\tau_{1}+h}\left(B\left(\nabla_{x} u(\tau)\right), 1\right)_{L^{2}} \mathrm{~d} \tau \\
& \leq-\int_{\tau_{1}}^{\tau_{2}}\left(\operatorname{div} b\left(\nabla_{x} u(\tau+h)\right), \frac{u(\tau+h)-u(\tau)}{h}\right)_{L^{2}} \mathrm{~d} \tau,
\end{aligned}
$$

where all of the integrals have sense due to (4.3) and assumption $u \in L^{\infty}\left(\mathbb{R}_{+}\right.$, $\left.W_{0}^{1, p}(\Omega)\right)$. Passing now to the limit $h \rightarrow 0$ and using the assumptions of the lemma, we deduce that, for almost all $\tau_{2}$ and $\tau_{1}$,

$$
\left(B\left(\nabla_{x} u\left(\tau_{2}\right)\right), 1\right)_{L^{2}}-\left(B\left(\nabla_{x} u\left(\tau_{1}\right)\right), 1\right)_{L^{2}}=-\int_{\tau_{1}}^{\tau_{2}}\left(\operatorname{div} b\left(\nabla_{x} u(\tau)\right), \partial_{\tau} u(\tau)\right)_{L^{2}} \mathrm{~d} \tau
$$

which shows that $\left(B\left(\nabla_{x} u\right), 1\right)_{L^{2}} \in A C([0, R])$ and (4.21) holds. Lemma 4.4 is proved.

Remark 4.5. The assertion of the lemma looks completely standard. However, in contrast to the standard situation, we cannot write that

$$
\left(\operatorname{div} b\left(\nabla_{x} u(\tau)\right), \partial_{\tau} u(\tau)\right)_{L^{2}}=-\left(b\left(\nabla_{x} u(\tau)\right), \partial_{\tau} \nabla_{x} u(\tau)\right)_{L^{2}}
$$

since $b\left(\nabla_{x} u\right) \notin L^{2}(\Omega)$. As a result, we cannot use the standard method of approximation of the function $u$ by smooth ones and have to use more delicate methods.

Corollary 4.6. Let the above assumptions hold. Then, any strong solution $u$ of problem (4.13) satisfies the energy identity

$$
\begin{aligned}
& \left(B\left(\nabla_{x} u\left(\tau_{2}\right)\right), 1\right)_{L^{2}}-\left(B\left(\nabla_{x} u\left(\tau_{1}\right)\right), 1\right)_{L^{2}} \\
& \quad+\int_{\tau_{1}}^{\tau_{2}}\left\|\partial_{\tau} u(\tau)\right\|_{L^{2}}^{2}+\left(\Psi\left(\partial_{\tau} u(\tau)\right), 1\right)_{L^{2}} \mathrm{~d} \tau \\
& =-\int_{\tau_{1}}^{\tau_{2}}\left(f_{\varepsilon}(\tau, u(\tau)), \partial_{\tau} u(\tau)\right)_{L^{2}} \mathrm{~d} \tau
\end{aligned}
$$

for all $0 \leq \tau_{1} \leq \tau_{2}$ 
Indeed, according to (4.17) and (4.7), we see that $\operatorname{div} b\left(\nabla_{x} u\right) \in L^{\infty}\left(\mathbb{R}_{+}, H^{-1}(\Omega)\right)$ since

$$
\operatorname{div} b\left(\nabla_{x} u(\tau)\right)=\Theta_{u}(\tau)-f_{\varepsilon}(\tau, u(\tau)) .
$$

Therefore, (4.21) holds. Multiplying then equation (4.13) by $\partial_{\tau} u$ and integrating over $\left[\tau_{1}, \tau_{2}\right] \times \Omega$, we deduce $(4.25)$

\subsection{Uniform estimates and existence of solutions}

The aim of this section is to deduce uniform with respect to $\varepsilon \rightarrow 0$ estimates for the solutions of problem (4.13) and prove the existence of a solution of that problem in the sense of Definition 4.3. To this end, we approximate the function $\Psi(v)$ by smooth ones $\Psi_{\delta}(v)$ with

$$
\Psi_{\delta}(v):=\int_{\mathbb{R}^{m}} \varphi(r / \delta) \Psi(v-r) \mathrm{d} r,
$$

where $\varphi$ is a standard smoothing kernel in $\mathbb{R}^{m}$, see Appendix A for the detailed study of the approximating functions $\Psi_{\delta}$, and consider the smoothed version of problem (4.13)

$$
\left\{\begin{array}{l}
\partial_{\tau} u+\Psi_{\delta}^{\prime}\left(\partial_{\tau} u\right)=\operatorname{div} b\left(\nabla_{x} u\right)-f_{\varepsilon}(\tau, u), \\
\left.u\right|_{\partial \Omega}=0, \quad u(0, \cdot)=u_{0} .
\end{array}\right.
$$

As in the semilinear case, our next task is to obtain uniform with respect to $\varepsilon$ and $\delta$ estimates for the solutions of the auxiliary equation (4.26). The required solution $u(\tau)$ of the initial problem (4.13) will be than obtained as a limit $\delta \rightarrow 0$.

We say that a function $u=u(\tau, x)$ is a solution of (4.26) if it satisfies all of the assertions of Definition 4.3 except of integral estimate (4.18) which should be replaced by

$$
\begin{aligned}
& \left\|\partial_{\tau} u\left(\tau_{2}\right)\right\|_{L^{2}}^{2}-\left\|\partial_{\tau} u\left(\tau_{1}\right)\right\|_{L^{2}}^{2}+2 \alpha \int_{\tau_{1}}^{\tau_{2}}\left\|\nabla_{x} \partial_{\tau} u(\tau)\right\|_{L^{2}}^{2} \mathrm{~d} \tau \\
& \quad+2 \int_{\tau_{1}}^{\tau_{2}}\left[\left(f_{\varepsilon, \tau}^{\prime}(\tau, u(\tau)), \partial_{\tau} u(\tau)\right)_{L^{2}}+\left(f_{\varepsilon, u}^{\prime}(\tau, u(\tau)) \partial_{\tau} u(\tau), \partial_{\tau} u(\tau)\right)_{L^{2}}\right] \mathrm{d} \tau \\
& \leq C \delta
\end{aligned}
$$

where the additional constant $C$ is independent of $\delta$ (and appears due to the fact that $\Psi_{\delta}(v)$ is not homogeneous of order one).

The next theorem gives the existence of such solutions.

Theorem 4.7. Let the above assumptions hold and let $u_{0} \in W^{1, p}(\Omega)$ and $\Theta_{u}(0) \in$ $L^{2}(\Omega)$. Then, for every $\delta>0$, there exists at least one solution $u=u_{\delta}$ in the sense 
of the above definition. Moreover, every such solution satisfies the energy identity

$$
\begin{aligned}
& \left(B\left(\nabla_{x} u\left(\tau_{2}\right)\right), 1\right)_{L^{2}}-\left(B\left(\nabla_{x} u\left(\tau_{1}\right)\right), 1\right)_{L^{2}} \\
& \quad+\int_{\tau_{1}}^{\tau_{2}}\left\|\partial_{\tau} u(\tau)\right\|_{L^{2}}^{2}+\left(\Psi_{\delta}^{\prime}\left(\partial_{\tau} u(\tau)\right), \partial_{\tau} u(\tau)\right)_{L^{2}} \mathrm{~d} \tau \\
& =-\int_{\tau_{1}}^{\tau_{2}}\left(f_{\varepsilon}(\tau, u(\tau)), \partial_{\tau} u(\tau)\right)_{L^{2}} \mathrm{~d} \tau
\end{aligned}
$$

for all $0 \leq \tau_{1} \leq \tau_{2}$ and the following dissipative estimate:

$$
\|u(\tau)\|_{W^{1, p}(\Omega)}+\int_{\tau}^{\tau+1}\left\|\partial_{\tau} u(s)\right\|_{L^{2}}^{2} d s \leq Q\left(\|u(0)\|_{W^{1, p}(\Omega)}\right) \mathrm{e}^{-k \tau}+C_{f},
$$

where $k, C_{F}>0$ and the monotone function $Q$ are independent of $\tau, \varepsilon$ and $\delta$.

Proof. Indeed, the energy identity follows from Lemma 4.4 exactly as in Corollary 4.6. Let us check the dissipative estimate. The energy equality together with the obvious formula $\frac{d}{d \tau}(F(\varepsilon \tau, u(\tau)), 1)_{L^{2}}=\left(f_{\varepsilon}(\tau, u(\tau)), \partial_{\tau} u(\tau)+\varepsilon\left(F_{t}^{\prime}(\varepsilon \tau, u(\tau)), 1\right)_{L^{2}}\right.$ gives

$$
\begin{aligned}
& \frac{d}{d \tau}\left(\left(b\left(\nabla_{x} u(\tau), 1\right)_{L^{2}}+(F(\varepsilon \tau, u(\tau)), 1)\right)_{L^{2}}+1 / 2\left\|\partial_{\tau} u(\tau)\right\|_{L^{2}}^{2}\right. \\
& \quad+\left(\Psi_{\delta}^{\prime}\left(\partial_{\tau} u(\tau)\right), \partial_{\tau} u(\tau)\right)_{L^{2}} \\
& =\varepsilon\left(F_{t}^{\prime}(\varepsilon \tau, u(\tau)), 1\right)_{L^{2}} .
\end{aligned}
$$

Furthermore, we multiply equation (4.26) by $u(\tau)$ and integrate by $x$. Then, using (4.3)(1) and the fact that $\Psi_{\delta}^{\prime}$ is uniformly bounded in $L^{\infty}$, we have

$$
\frac{d}{d \tau}\|u(\tau)\|_{L^{2}}^{2}+\alpha\|\nabla u(\tau)\|_{L^{p}(\Omega)}^{p}+2\left(f_{\varepsilon}(\tau, u(\tau)), u(\tau)\right)_{L^{2}} \leq C,
$$

where $C$ is independent of $\tau, \varepsilon$ and $\delta$. Multiplying estimate (4.31) by $2 L$ where $L$ is a sufficiently large positive number, taking a sum with equation (4.30), using (2.7) and the fact that $\Psi_{\delta}^{\prime} \leq C$, see Lemma A.2, we have

$$
\frac{d}{d \tau} E(\tau, u(\tau))+\theta\|u(\tau)\|_{W^{1, p}(\Omega)}^{p} \leq C_{1},
$$

where

$$
E(\tau, v):=L\|v\|_{L^{2}}^{2}+\left(B\left(\nabla_{x} v\right), 1\right)_{L^{2}}+(F(\varepsilon \tau, v), 1)_{L^{2}}
$$

and positive constants $\theta$ and $C_{1}$ are independent of $\varepsilon, \delta$ and $\tau$. Moreover, due to (2.6) and (4.4), we have

$$
\rho\|v\|_{W^{1, p}(\Omega)}^{p}-C \leq E(\tau, v) \leq Q\left(\|v\|_{W^{1, p}(\Omega)}\right)
$$


for some positive $\rho$ and monotone function $Q$. Estimate (4.29) follows now from (4.32) and (4.34) and the Gronwall-type estimate (see [3, Lem. 2.7]). Thus, the dissipative estimate (4.29) is proved.

To verify the existence of solutions, we use the Galerkin approximation method. Indeed, let $P_{N}$ be the orthoprojectors associated with a smooth complete orthonormal system in $L^{2}(\Omega)$ which is assumed to be complete in $W_{0}^{1, p}(\Omega)$ and let $u_{N}(t) \in P_{N} L^{2}(\Omega)$ be a solution of the following system of ODEs

$$
P_{N}\left(\partial_{\tau} u_{N}+\Psi_{\delta}^{\prime}\left(\partial_{\tau} u_{N}\right)\right)=P_{N} \operatorname{div} b\left(\nabla_{x} u_{N}(\tau)\right)-P_{N} f_{\varepsilon}\left(\tau, u_{N}\right) .
$$

We complete this system of equations by the initial value $u_{N}^{0}=u_{N}(0)$ constructed as follows: let $\Theta_{N}^{0} \in P_{N} L^{2}(\Omega)$ be some sequence converging strongly to $\Theta_{u}(0)$ in $L^{2}(\Omega)$ and let $\tilde{u}_{N}^{0} \in P_{N} W_{0}^{1, p}(\Omega)$ be a sequence converging to $u(0)$, then we find $w=u_{N}^{0} \in P_{N} W_{0}^{1, p}(\Omega)$ as a solution of the following equation

$$
-M \tilde{u}_{N}^{0}+\Theta_{N}^{0}=P_{N} \operatorname{div} b\left(\nabla_{x} v\right)-P_{N} f_{\varepsilon}(0, v)-M v,
$$

where the (large) positive constant $M$ will be chosen in the next lemma.

Lemma 4.8. Let the above assumptions hold. Then, there exists a positive M such that, for every $N$, equation (4.36) has a unique solution $u_{N}^{0}$ and this solution tends to $u(0)$ as $N \rightarrow \infty$ in the space $W_{0}^{1, p}(\Omega)$.

Proof. Let the sequences $\tilde{u}_{N}^{0}$ and $\Theta_{N}^{0}$ be fixed. We first deduce the a priori estimate

$$
\|v\|_{W^{1, p}(\Omega)} \leq C
$$

for the solution $v$ of (4.36), where the constant $C$ is independent of $M$ and $N$. To this end, we set $\bar{w}:=v-\tilde{u}_{N}^{0}$ and multiply equation (4.36) by $\bar{w}$ and obtain that

$$
\left(b\left(\nabla_{x} \bar{w}+\nabla_{x} \tilde{u}_{N}^{0}\right), \nabla_{x} \bar{w}\right)_{L^{2}}+\left(f_{\varepsilon}\left(0, \bar{w}+\tilde{u}_{N}^{0}\right), \bar{w}\right)_{L^{2}}+M\|\bar{w}\|_{L^{2}}^{2}+\left(\Theta_{N}^{0}, \bar{w}\right)_{L^{2}}=0 .
$$

Using now assumptions (4.3) for the function $b$ and the fact that the sequence $\tilde{u}_{N}^{0}$ is uniformly bounded in $W^{1, p}(\Omega)$, we see that $\left(b\left(\nabla_{x} \bar{w}+\nabla_{x} \tilde{u}_{N}^{0}\right), \nabla_{x} \bar{w}\right)_{L^{2}} \geq$ $\alpha\|v\|_{W^{1, p}(\Omega)}^{p}-C$, where $\alpha>0$ and $C$ are independent of $N$ and $M$. Analogously, using assumptions (2.6) and (2.5) for the function $f$ and the embed$\operatorname{ding} W^{1, p} \subset L^{r_{\max }+2}$ (the subordination assumption), we see that $\left(f_{\varepsilon}(0, \bar{w}+\right.$ $\left.\left.\tilde{u}_{N}^{0}\right), \bar{w}\right)_{L^{2}} \geq \beta\|v\|_{L^{r+2}(\Omega)}^{r+2}-C_{1}$ for some $\beta, C_{1}>0$ which are also independent of $N$ and $M$. Inserting these estimates into (4.38) and using that $\Theta_{N}^{0}$ is also uniformly bounded in $L^{2}(\Omega)$, we deduce a priori estimate (4.37).

To finish the proof of the lemma, we verify the existence of a solution for (4.36) in a standard way using a priori estimate (4.37). Let us verify the uniqueness. 
Indeed, let $v_{1}$ and $v_{2}$ be two solutions of (4.36) and let $w=v_{1}-v_{2}$. Then, this function solves

$$
P_{N} \operatorname{div}\left[b\left(\nabla_{x} v_{1}\right)-b\left(\nabla_{x} v_{2}\right)\right]-P_{N}\left[f_{\varepsilon}\left(0, v_{1}\right)-f_{\varepsilon}\left(0, v_{2}\right)\right]-M w=0 .
$$

Multiplying this equation by $w$, integrating over $x$ and using assumption (4.3)(3) and estimate (4.9) together with the uniform estimate (4.37) for $v_{1}$ and $v_{2}$, we see that

$$
\kappa\|w\|_{H^{1}}^{2}-K\|w\|_{W^{1-\gamma, 2}(\Omega)}^{2}+M\|w\|_{L^{2}}^{2} \leq 0,
$$

where positive constants $K$ and $\kappa$ are independent of $M$ and $\gamma>0$ is the same as in (4.9) and $N$. Together with the standard interpolation estimate, this estimate implies $w \equiv 0$ if $M>M_{0}(\kappa, \gamma, K)$. Thus, uniqueness is also verified.

Finally, since the sequence of solutions $v=u_{N}^{0}$ of problem (4.36) is uniformly bounded in $W_{0}^{1, p}(\Omega)$, we can assume, without loss of generality, that it converges weakly to some function $u_{0} \in W_{0}^{1, p}(\Omega)$ which, by standard arguments, should satisfy the limit equation

$$
-M u(0)+\Theta_{u}(0)=\operatorname{div} b\left(\nabla_{x} u_{0}\right)-f_{\varepsilon}\left(0, u_{0}\right)-M u_{0} .
$$

Since, by the definition of $\Theta_{u}(0)$, the function $u(0)$ solves this equation, then, necessarily, $u_{0}=u(0)$. The strong convergence of $u_{N}^{0}$ can be proved by the standard monotonicity arguments. Lemma 4.8 is proved.

We are now ready to finish the proof of the theorem. To this end, we note that, multiplying equation (4.35) by $\partial_{\tau} u_{N}+2 L u_{N}$ and arguing as before, we can prove that the solution $u_{N}(t)$ of (4.35) satisfies the analog of (4.28) and (4.29) (uniformly with respect to $N)$. Since (4.35) is, in a fact, a system of ODEs, this a priori estimate is sufficient to establish the global solvability of the Galerkin approximation system. Moreover, since the sequence $u_{N}^{0}$ is also uniformly bounded, the dissipative estimate (4.29) shows that

$$
\left\|u_{n}(\tau)\right\|_{W^{1, p}(\Omega)}+\int_{\tau}^{\tau+1}\left\|\partial_{\tau} u_{N}(r)\right\|_{L^{2}}^{2} \mathrm{~d} r \leq C,
$$

where the constant $C$ is independent of $\tau$ and $N$.

We now derive estimates for $\partial_{\tau} u$ and estimate (4.27). To this end, we differentiate equation (4.35) by $\tau$, denote $v_{N}(\tau)=\partial_{\tau} u_{N}(\tau)$ and multiply it by $v_{N}(\tau)$. Then, we have

$$
\begin{aligned}
& \frac{d}{d \tau}\left[\left(\Psi_{\delta}^{\prime}\left(v_{N}\right) \cdot v_{N}-\Psi_{\delta}\left(v_{N}\right), 1\right)_{L^{2}}+1 / 2\left\|v_{N}\right\|_{L^{2}}^{2}\right] \\
& \quad+\left(b^{\prime}\left(\nabla_{x} u_{N}\right) \nabla_{x} v_{N}, \nabla_{x} v_{N}\right)_{L^{2}}+\left(f_{\varepsilon, \tau}^{\prime}\left(\tau, u_{N}\right), v_{N}\right)_{L^{2}}+\left(f_{\varepsilon, u}^{\prime}\left(\tau, u_{N}\right) v_{N}, v_{N}\right)_{L^{2}} \\
& =0
\end{aligned}
$$


Integrating this equality by $\tau \in\left[\tau_{1}, \tau_{2}\right]$ and using (4.3)(3) and (A.7), we deduce the analog of (4.27) for $u_{N}$ :

$$
\begin{aligned}
& \left\|\partial_{\tau} u_{N}\left(\tau_{2}\right)\right\|_{L^{2}}^{2}-\left\|\partial_{\tau} u_{N}\left(\tau_{1}\right)\right\|_{L^{2}}^{2}+2 \kappa \int_{\tau_{1}}^{\tau_{2}}\left\|\nabla_{x} \partial_{\tau} u_{N}(\tau)\right\|_{L^{2}}^{2} \mathrm{~d} \tau \\
& +2 \int_{\tau_{1}}^{\tau_{2}}\left[\left(f_{\varepsilon, \tau}^{\prime}\left(\tau, u_{N}(\tau)\right), \partial_{\tau} u_{N}(\tau)\right)_{L^{2}}+\left(f_{\varepsilon, u}^{\prime}\left(\tau, u_{N}(\tau)\right) \partial_{\tau} u_{N}(\tau), \partial_{\tau} u_{N}(\tau)\right)_{L^{2}}\right] \mathrm{d} \tau \\
& \leq C \delta
\end{aligned}
$$

with the constant $C$ independent of $N$. Moreover, using estimates (4.7) and (4.9) together with (4.39), we deduce from (4.40) that

$$
\frac{d}{d \tau}\left[2\left(\Psi_{\delta}^{\prime}\left(v_{N}\right) \cdot v_{N}-\Psi_{\delta}\left(v_{N}\right), 1\right)_{L^{2}}+\left\|v_{N}\right\|_{L^{2}}^{2}\right]+\kappa\left\|\nabla_{x} v_{N}\right\|_{L^{2}}^{2} \leq C\left(1+\left\|v_{N}\right\|_{W^{1-\gamma, 2}(\Omega)}^{2}\right) .
$$

Applying the Gronwall estimate to this relation and using (A.7), interpolation estimate $\|\cdot\|_{W^{1-\gamma, 2}} \leq C\|\cdot\|_{L^{2}}^{\gamma}\|\cdot\|_{H^{1}}^{1-\gamma}$ and estimate (4.39) for estimating the integral over the $L^{2}$-norm of $v$, we will have

$$
\left\|v_{N}(\tau)\right\|_{L^{2}}^{2}+\int_{\tau}^{\tau+1}\left\|\nabla_{x} v_{N}(r)\right\|_{L^{2}}^{2} \mathrm{~d} r \leq C\left(1+\left\|v_{N}(0)\right\|_{L^{2}}^{2}\right),
$$

where $C$ is independent of $N$. Furthermore, $v_{N}(0)=\partial_{t} u_{N}(0)$ can be found from

$$
P_{N}\left[v_{N}(0)+\Psi_{\delta}^{\prime}\left(v_{N}(0)\right)\right]=\Theta_{u_{N}}(0):=P_{N}\left[\operatorname{div} b\left(\nabla_{x} u_{N}(0)\right)-f_{\varepsilon}\left(0, u_{N}(0)\right)\right] .
$$

But, according to (4.36)

$$
\Theta_{u_{N}}(0)=\Theta_{N}^{0}+M\left(u_{N}(0)-\tilde{u}_{N}^{0}\right)
$$

and, consequently, $\Theta_{u_{N}}(0)$ is uniformly bounded in $L^{2}(\Omega)$ and tends strongly as $N \rightarrow \infty$ to $\Theta_{u}(0)$. ¿From equation (4.44) and the monotonicity of $\Psi_{\delta}^{\prime}$, we conclude that the same is true for the functions $v_{N}(0)=\partial_{\tau} u(0)$ as well. Thus, estimate (4.43) gives

$$
\left\|v_{N}(\tau)\right\|_{L^{2}}^{2}+\int_{\tau}^{\tau+1}\left\|\nabla_{x} v_{N}(r)\right\|_{L^{2}}^{2} \mathrm{~d} r \leq C_{1},
$$

where the constant $C_{1}$ is independent of $\tau$ and $N$.

Finally, the uniform estimates (4.39) and (4.45) allow to pass in a standard way to the weak limit $N \rightarrow \infty$ in equations (4.35) and to obtain a solution $u(\tau)$ of the limit problem (4.26) belonging to the class (4.17). Moreover, the monotonicity arguments (see e.g. the proof of the next theorem) show that $\partial_{\tau} u_{N}$ converges to 
$\partial_{\tau} u$ strongly in $L^{2}([0, R] \times \Omega)$. This convergence, together with Lemma 4.1 (see (4.7) and (4.9)) allow to pass to the limit $N \rightarrow \infty$ in estimate (4.41) and verify that (4.27) is satisfied for almost all $\tau_{1}$ and $\tau_{2}$. Thus, Theorem 4.7 is proved.

Our next task is to construct solutions for (4.13) by passing to the limit $\delta \rightarrow 0$ in (4.26).

Theorem 4.9. Let the above assumptions hold. Then, problem (4.13) possesses at least one solution $u$ in the sense of Definition 4.3. Every such solution satisfies the dissipative estimate (4.29) (uniformly with respect to $\varepsilon$ ) and the energy equality (4.25).

Proof. Indeed, the energy equality and dissipative estimate for the solution $u$ of problem (4.13) can be verified exactly as in the previous theorem. So, it remains to check existence.

We will construct the required solution $u(\tau)$ of problem (4.13) as a limit $\delta \rightarrow 0$ of the solutions $u_{\delta}(\tau)$ of the regularized problems (4.26). Indeed, using the dissipative estimate (4.29) and estimate (4.27), we see that the sequence $u_{\delta}$ is uniformly bounded in $L^{\infty}\left(\mathbb{R}_{+}, W_{0}^{1, p}(\Omega)\right)$. Moreover, using (4.27) and the Gronwall estimate (analogously to (4.43)), we see that the corresponding sequence of derivatives $\partial_{\tau} u_{\delta}$ is uniformly bounded in $L^{\infty}\left([0, R], L^{2}(\Omega)\right) \cap L^{2}\left([0, R], H_{0}^{1}(\Omega)\right)$, for every $R>0$. Thus, without loss of generality, we can assume that there exists a weak limit $u=\lim _{n \rightarrow \infty} u_{\delta_{n}}$ where $\delta_{n} \rightarrow 0$ as $n \rightarrow \infty$ and $u_{n}=u_{\delta_{n}}$ solves (4.26) with $\delta=\delta_{n}$. To be more precise,

$$
\begin{aligned}
& u_{n} \rightarrow u \text { weakly* in } \\
& L^{\infty}\left([0, R], W^{1, p}(\Omega)\right) \cap W^{1, \infty}\left([0, R], L^{2}(\Omega)\right) \cap H^{1}\left([0, R], H_{0}^{1}(\Omega)\right) .
\end{aligned}
$$

Let us prove that $u$ solves (4.13). To this end, we set

$$
v_{n}:=\partial_{\tau} u_{n}, \quad \Theta_{n}:=\Theta_{u_{n}}=\Psi_{\delta_{n}}^{\prime}\left(v_{n}\right)+v_{n}
$$

Since the sequence $\partial_{t} u_{n}$ is uniformly bounded in $L^{\infty}\left([0, R], L^{2}(\Omega)\right)$ and $\Psi_{\delta}^{\prime}$ is bounded in $L^{\infty}$, the sequence $\Theta_{n}$ is uniformly bounded in $L^{\infty}\left([0, R], L^{2}(\Omega)\right)$. Consequently, without loss of generality, we can assume that $\Theta_{n} \rightarrow \Theta$ weakly* in that space. We need to prove that

$$
\text { (1) } \Theta=\Theta_{u}:=\operatorname{div} B^{\prime}\left(\nabla_{x} u\right)-f_{\varepsilon}(\tau, u), \quad \text { (2) } \Theta \in \partial \Psi(v)+v
$$

where $v=\partial_{\tau} u$. To verify (1) we let $\mathcal{B}(u):=-\operatorname{div}\left(b\left(\nabla_{x} u\right)\right)$. Then, due to assumptions (4.3), this operator is monotone as the operator from the space $W:=$ $L^{p}\left([0, R], W_{0}^{1, p}(\Omega)\right)$ to $W^{*}$. Moreover, this operator is maximal monotone in $W$ as a derivative of a convex functional, see e.g., [29]. Let now $\psi_{n}:=\mathcal{B}\left(u_{n}\right)$. Then, $\psi_{n}$ is uniformly bounded in $W^{*}$ and, consequently, $\psi_{n} \rightarrow \psi$ weakly in $W^{*}=L^{q}\left([0, R], W^{-1, q}(\Omega)\right)$. Taking in mind that the operator $f_{\varepsilon}$ is subordinated 
to $\mathcal{B}$ (see Lemma 4.1 and estimate (4.7)) and the functions $u_{n}$ converge strongly in $L^{p}\left([0, R], W^{1-\beta, p}(\Omega)\right)$, for any $\beta>0$, we have

$$
\Theta+\psi+f_{\varepsilon}(\tau, u)=0 \text {. }
$$

On the other hand, multiplying equation (4.26) by $u_{n}$, we have

$$
\left.\left(\mathcal{B}\left(u_{n}\right), u_{n}\right)\right)_{L^{2}([0, R] \times \Omega)}=-\left(\Theta_{n}, u_{n}\right)_{L^{2}([0, R] \times \Omega)}-\left(f_{\varepsilon}\left(\tau, u_{n}\right), u_{n}\right)_{L^{2}([0, R] \times \Omega)} .
$$

Passing to the limit in the right-hand side of (4.50) and using again that $f_{\varepsilon}$ is subordinated, we deduce

$$
\lim _{n \rightarrow \infty}\left(\mathcal{B}\left(u_{n}\right), u_{n}\right)=-(\Theta, u)-\left(f_{\varepsilon}(\tau, u)=(\psi, u) .\right.
$$

Since $\mathcal{B}$ is maximal monotone, the last equality implies $\mathcal{B}(u)=\psi$, and (4.48)(1) is verified.

The inclusion (4.48)(2) can be checked exactly as in the proof of Theorem 2.9. Thus, $u$ solves indeed the limit problem (4.13).

Furthermore, to verify that $u$ is a solution of (4.13) in the sense of Definition 4.3, we want to pass to the limit in estimate (4.27) for $u_{n}$, thus needing the strong convergence

$$
u_{n} \rightarrow u \text { strongly in } L^{p}\left([0, R], W^{1, p}(\Omega) \cap H^{1}\left([0, R], L^{2}(\Omega)\right) .\right.
$$

To this end, we rewrite energy equality (4.28) in the form

$$
\begin{aligned}
\left\langle\Psi_{\delta_{n}}\left(v_{n}\right), 1\right\rangle_{R} & +\left\langle\left|v_{n}\right|^{2}, 1\right\rangle_{R}+\left(B\left(\nabla_{x} u_{n}(R)\right), 1\right)_{L^{2}} \\
& =\left(B\left(\nabla_{x} u(0)\right), 1\right)_{L^{2}}-\left\langle P_{\delta_{n}}\left(v_{n}\right), 1\right\rangle_{R}-\left\langle f_{\varepsilon}\left(\tau, u_{n}\right), v_{n}\right\rangle_{R},
\end{aligned}
$$

where $P_{\delta}(v):=\Psi_{\delta}^{\prime}(v) \cdot v-\Psi_{\delta}(v)$, see Appendix A.

Using the fact that $f_{\varepsilon}$ is relatively compact and estimate (A.7) and passing to the limit $n \rightarrow \infty$, we get

$$
\begin{aligned}
\lim _{n \rightarrow \infty}\left\langle\Psi_{\delta_{n}}\left(v_{n}\right), 1\right\rangle_{R}+\left\langle\left|v_{n}\right|^{2}, 1\right\rangle_{R} & +\left(B\left(\nabla_{x} u_{n}(R)\right), 1\right)_{L^{2}} \\
& =\left(B\left(\nabla_{x} u(0)\right), 1\right)_{L^{2}}-\left\langle f_{\varepsilon}(\tau, u), v\right\rangle_{R}
\end{aligned}
$$

which together with the energy equality for the limit equation (4.13) gives

$$
\begin{aligned}
\lim _{n \rightarrow \infty}\left[\left\langle\Psi\left(v_{n}\right), 1\right\rangle_{R}+\langle|\right. & \left.\left.\left.v_{n}\right|^{2}, 1\right\rangle_{R}+\left(B\left(\nabla_{x} u_{n}(R)\right), 1\right)_{L^{2}}\right] \\
& =\langle\Psi(v), 1\rangle_{R}+\left\langle|v|^{2}, 1\right\rangle_{R}+\left(B\left(\nabla_{x} u(R)\right), 1\right)_{L^{2}} .
\end{aligned}
$$


Since the functions $\Psi$ and $B$ are convex, equality (4.55) implies

$$
\begin{aligned}
& \text { 1) } \lim _{n \rightarrow \infty}\left\langle\left|v_{n}\right|^{2}, 1\right\rangle_{R}=\left\langle|v|^{2}, 1\right\rangle_{R}, \\
& \text { 2) } \lim _{n \rightarrow \infty}\left(B\left(\nabla_{x} u_{n}(R)\right), 1\right)_{L^{2}}=\left(B\left(\nabla_{x} u(R)\right), 1\right)_{L^{2}}
\end{aligned}
$$

Since the spaces $L^{2}$ and $W^{1, p}$ are uniformly convex, $B$ is strictly convex and $R>0$ is arbitrary, then (4.56) implies (4.52).

It is now not difficult to pass to the limit in (4.27). Indeed, the strong convergence (4.52) together with weak convergence (4.46) imply that $u_{n} \rightarrow u$ strongly in $L^{s}\left([0, R], W^{1, p}(\Omega)\right), \partial_{\tau} u_{n} \rightarrow \partial_{\tau} u$ strongly in $L^{2}\left([0, R], W^{1-\gamma}(\Omega)\right)$, for every $s<$ $\infty$ and $\gamma>0$ and $\left\|\partial_{\tau} u_{n}(\tau)\right\|_{L^{2}} \rightarrow\left\|\partial_{\tau} u(\tau)\right\|_{L^{2}}$ for almost all $\tau \in \mathbb{R}_{+}$. These convergence, together with Lemma 4.1 justify the passing to the limit $\delta \rightarrow 0$ in (4.27) and show that the solution $u$ satisfies (4.18). Thus, Theorem 4.9 is proved.

Analogously to the semilinear case, estimate (4.18) and energy equality allow to deduce the crucial estimate of $\partial_{\tau} u$ in the space $L^{2}\left([0, R], H^{1}(\Omega)\right)$. Next corollary is the analog of the dissipation integral (2.53) for solutions of problem (4.13).

Corollary 4.10. Let the assumptions of Theorem 4.9 hold and let $u$ be a solution of problem (4.13). Then, for every $\tau_{1}, \tau_{2} \in \mathbb{R}_{+}, \tau_{2} \geq \tau_{1}$, one has

$$
\int_{\tau_{1}}^{\tau_{2}}\left\|\partial_{\tau} u(\tau)\right\|_{L^{1}(\Omega)}+\left\|\partial_{\tau} u(\tau)\right\|_{L^{2}}^{2} \mathrm{~d} \tau \leq Q\left(\|u(0)\|_{W^{1, p}(\Omega)}\right)\left[1+\varepsilon\left(\tau_{2}-\tau_{1}\right)\right],
$$

where the monotone function $Q$ is independent of $\varepsilon, \tau_{1}$ and $\tau_{2}$.

Proof. In order to obtain estimate (4.57), it is sufficient to integrate the energy equality (4.25) and use the uniform bounds for the $W^{1, p}$-norm of the solution obtained above. Indeed, integrating (4.25) by $\tau \in\left[\tau_{1}, \tau_{2}\right]$ and using (4.29) and (4.8) and the obvious equality $f_{\varepsilon}(\tau, u) . \partial_{\tau} u=\partial_{\tau} F(\varepsilon \tau, u)-\varepsilon F_{t}^{\prime}(\varepsilon \tau, u)$, we get

$$
\begin{aligned}
\int_{\tau_{1}}^{\tau_{2}}\left(\Psi\left(\partial_{\tau} u(\tau)\right), 1\right)_{L^{2}}+1 / 2\left\|\partial_{\tau} u(\tau)\right\|_{L^{2}}^{2} \mathrm{~d} \tau & \leq Q\left(\left\|u_{0}\right\|_{W^{1, p}(\Omega)}\right) \\
& +\int_{\tau_{1}}^{\tau_{2}} \varepsilon\left(F_{t}^{\prime}(\varepsilon \tau, u(\tau)), 1\right)_{L^{2}} \mathrm{~d} \tau .
\end{aligned}
$$

Using estimates (4.8) and (4.29) in order to estimate the integral with $F_{t}^{\prime}$ and estimate $(2.3)(1)$ and for estimating the term with $\Psi$, we obtain the required estimate (4.57) and finish the proof of Corollary 4.10.

Next proposition is the analog of Proposition 2.11. 
Proposition 4.11. Let the assumptions of Section 4.1 hold and let $u=u(\tau)$ be a solution of problem (4.13) in the sense of Definition 4.3. Then, the following estimate holds:

$$
\int_{\tau_{1}}^{\tau_{2}}\left\|\partial_{\tau} u(\tau)\right\|_{H^{1}} \mathrm{~d} \tau \leq C\left(1+\varepsilon\left(\tau_{2}-\tau_{1}\right)\right),
$$

where the constant $C$ depends on the appropriate norms of $u(0)$ and $\Theta_{u}(0)$, but is independent of $\varepsilon>0, \tau_{1} \geq 0$ and $\tau_{2} \geq \tau_{1}$.

Proof. Indeed, since $f_{\varepsilon, \tau}^{\prime}(\tau, u)=\varepsilon f_{t}^{\prime}(\varepsilon \tau, u)$, then using (4.7), (4.9) and the dissipative estimate (4.29), one can transform estimate (4.18) as follows:

$$
\begin{aligned}
\left\|\partial_{\tau} u\left(\tau_{2}\right)\right\|_{L^{2}}^{2} & -\left\|\partial_{\tau} u\left(\tau_{1}\right)\right\|_{L^{2}}^{2} \\
& \quad+\kappa \int_{\tau_{1}}^{\tau_{2}}\left\|\nabla_{x} \partial_{\tau} u(r)\right\|_{L^{2}}^{2} \mathrm{~d} r \leq C\left(\varepsilon^{2}+\int_{\tau_{1}}^{\tau_{2}}\left\|\partial_{\tau} u(r)\right\|_{L^{2}}^{2} \mathrm{~d} r\right),
\end{aligned}
$$

where the constant $C$ depends only on the $W^{1, p}$-norm of $u(0)$. The rest of the proof repeats word by word the proof of Proposition 2.11 and, by this reason, is omitted.

Corollary 4.12. Let the above assumptions hold and let $u=u(\tau)$ be a solution of problem (4.13). Then, the following estimate holds:

$$
\left\|\partial_{\tau} u(\tau)\right\|_{L^{2}}+\left\|\Theta_{u}(\tau)\right\|_{L^{2}} \leq Q\left(\left\|\Theta_{u}(0)\right\|_{L^{2}}+\|u(0)\|_{W^{1, p}(\Omega)}\right) \mathrm{e}^{-\alpha \tau}+C_{F},
$$

where the positive constants $C_{F}$ and $\alpha$ and the monotone function $Q$ are independent of $\tau, u$ and $\varepsilon$.

Proof. Indeed, in order to obtain the required estimate for $\partial_{\tau} u(\tau)$ it is sufficient to use estimate (4.60) with $\tau_{1}=0, \tau_{2}=\tau$, apply estimate (B.2) with $\phi(s):=$ $\mathrm{e}^{\alpha(s-\tau)}$ to it and use the dissipation integral (4.57) for estimating the integral in the right-hand side. Analogous estimate for $\Theta_{u}(\tau)$ follows from the obvious relation $\Theta_{u}(\tau) \in \partial_{\tau} u(\tau)+\Psi\left(\partial_{\tau} u(\tau)\right)$ and the fact that $\|\Psi(v)\|_{L^{\infty}} \leq C$ which, in turns, implies that

$$
\left\|\partial_{\tau} u(\tau)\right\|_{L^{2}} \leq\left\|\Theta_{u}(\tau)\right\|_{L^{2}} \leq\left\|\partial_{\tau} u(\tau)\right\|_{L^{2}}+C .
$$

Thus, Corollary 4.12 is proved.

We are now able to return back to the variable $t=\varepsilon \tau$ and the associated equation (4.1) and summarize the obtained results in the following theorem.

Theorem 4.13. Let the assumptions of Section 4.1 hold. Then, for all $\varepsilon>0$, there exists at least one solution u of problem (4.1) (such that $\tilde{u}(\tau):=u(\varepsilon \tau)$ is a solution 
of the scaled problem (4.13) in the sense of Definition 4.3). Every such solution satisfies the following dissipative estimate:

$$
\begin{aligned}
\|u(t)\|_{W^{1, p}(\Omega)}+\varepsilon \| \partial_{t} u(t) & \left\|_{L^{2}}+\right\| \Theta_{u}(0) \|_{L^{2}} \\
& \leq Q\left(\|u(0)\|_{W^{1, p}(\Omega)}+\left\|\Theta_{u}(0)\right\|_{L^{2}}\right) \mathrm{e}^{-\alpha t / \varepsilon}+C_{f},
\end{aligned}
$$

where the positive constants $\alpha$ and $C_{f}$ and monotone function $Q$ are independent of $\varepsilon$ and $t$. Moreover, the time derivative of that solution satisfies:

$$
\int_{T}^{T+1}\left\|\partial_{t} u(t)\right\|_{H^{1}} \mathrm{~d} t \leq C,
$$

where $C$ depends on $\|u(0)\|_{W^{1, p}(\Omega)}$ and $\left\|\Theta_{u}(0)\right\|_{L^{2}}$, but is independent of $\varepsilon$ and $T \in \mathbb{R}_{+}$.

Indeed, estimate (4.63) is an immediate corollary of estimates (4.29) and (4.61) and estimates (4.64) follow from Proposition 4.11 taking into the account that the $L^{1}\left(H^{1}\right)$-norm of $\partial_{t} u$ is scaling invariant.

\subsection{The vanishing-viscosity limit}

The aim of this section is to clarify the behavior of solutions of (4.1) as $\varepsilon \rightarrow 0$. In order to do so, exactly as in the semilinear case, we introduce the scaled time variable

$$
s(t):=t+\int_{0}^{t}\left\|\partial_{t} u_{\varepsilon}(r)\right\|_{L^{2}} \mathrm{~d} r,
$$

where $u_{\varepsilon}(t)$ solves (4.1) and rewrite equation (4.1) with respect to new dependent variable $\tilde{u}_{\varepsilon}(s):=u\left(t_{\varepsilon}(s)\right.$ ) (here and below $t_{\varepsilon}(s)$ denotes the inverse function to $s(t))$. Moreover, for simplifying the notations, we will write again $u(s)$ instead of $\tilde{u}(s)$. With respect to the new variables $u_{\varepsilon}(s)$ and $t_{\varepsilon}(s)(4.1)$ reads

$$
\left\{\begin{array}{l}
\operatorname{div}\left(b\left(\nabla_{x} u_{\varepsilon}(s)\right)\right)-f\left(t_{\varepsilon}(s), u_{\varepsilon}(s)\right) \in \partial \Psi\left(\partial_{s} u_{\varepsilon}(s)\right)+\varepsilon \frac{\partial_{s} u_{\varepsilon}(s)}{1-\left\|\partial_{s} u_{\varepsilon}(s)\right\|_{L^{2}}}, \\
t_{\varepsilon}^{\prime}(s)+\left\|\partial_{s} u_{\varepsilon}(s)\right\|_{L^{2}}=1, \quad t_{\varepsilon}(0)=0 \\
u_{\varepsilon}(0)=u_{0} .
\end{array}\right.
$$

In particular, this shows that

$$
\left\|\partial_{s} u(s)\right\|_{L^{2}}<1 \text { for almost all } s \geq 0 .
$$

Moreover, using the convex functional $\mathcal{C}_{\varepsilon}: L^{2}(\Omega) \rightarrow \mathbb{R}_{+}$given by (2.70), system (4.66) can be rewritten in the equivalent form:

$$
\left\{\begin{array}{l}
\partial \Psi\left(\partial_{s} u_{\varepsilon}(s)\right)+\partial \mathcal{C}_{\varepsilon}\left(\partial_{s} u_{\varepsilon}(s)\right) \ni \operatorname{div}\left(b\left(\nabla_{x} u_{\varepsilon}(s)\right)\right)-f\left(t_{\varepsilon}(s), u_{\varepsilon}(s)\right), \\
t_{\varepsilon}^{\prime}(s)+\left\|\partial_{s} u_{\varepsilon}(s)\right\|_{L^{2}}=1, \quad t_{\varepsilon}(0)=0 \\
u_{\varepsilon}(0)=u_{0}
\end{array}\right.
$$


Furthermore, since $\partial \mathcal{C}_{\varepsilon}$ converges to the limit operator $\partial \mathcal{C}_{0}$ defined by (2.73), we expect that the solutions $\left(u_{\varepsilon}, t_{\varepsilon}\right)$ converge, in the proper sense, to the solution $(u, t)$ of the limit problem

$$
\left\{\begin{array}{l}
\partial \Psi\left(\partial_{s} u(s)\right)+\partial \mathcal{C}_{0}\left(\partial_{s} u(s)\right) \ni \operatorname{div}\left(b\left(\nabla_{x} u(s)\right)\right)-f(t(s), u(s)), \\
t^{\prime}(s)+\left\|\partial_{s} u(s)\right\|_{L^{2}}=1, \quad t(0)=0 \\
u(0)=u_{0}
\end{array}\right.
$$

The rest of this section is devoted to the justification of the passage from (4.68) to (4.69). To this end, analogously to Lemma 2.15 , we first need to control new time $s=s_{\varepsilon}(t)$.

Lemma 4.14. Let the assumptions of Section 4.1 hold and let $u=u_{\varepsilon}(t)$ be a solution of (4.1) in the sense of Theorem 4.13. Then, the scaled time s satisfies the estimate

$$
s \geq t_{\varepsilon}(s) \geq \beta s-C, \quad t_{\varepsilon}(0)=0, \quad t_{\varepsilon}^{\prime}(s) \geq 0
$$

for some positive constants $\beta$ and $C$.

Indeed, estimate (4.70) follows immediately from the definition (4.65) of the scaled time $s$ and the uniform estimate (4.64) for the integral of $\partial_{t} u(t)$.

The next lemma interprets the uniform estimates obtained in the previous section in terms of the solutions $\left(u_{\varepsilon}(s), t_{\varepsilon}(s)\right)$ of problem (4.68).

Lemma 4.15. Let the above conditions hold. Then, the solutions $\left(u_{\varepsilon}, t_{\varepsilon}\right)$ of problems (4.68) satisfy the following estimates:

$$
\begin{aligned}
& \left\|u_{\varepsilon}(s)\right\|_{W^{1, p}(\Omega)}+\left\|\Theta_{u_{\varepsilon}}(s)\right\|_{L^{2}} \leq C=C\left(\left\|u_{0}\right\|_{W^{1, p}(\Omega)},\left\|\Theta_{u}(0)\right\|_{L^{2}}\right), \\
& \int_{S}^{S+1}\left\|\partial_{s} u_{\varepsilon}(s)\right\|_{H^{1}} \mathrm{~d} s \leq C, \\
& s \geq t_{\varepsilon}(s) \geq \beta s-C, \quad 1 \geq t_{\varepsilon}^{\prime}(s) \geq 0,
\end{aligned}
$$

where the positive constants $C$ and $\beta$ are independent of $\varepsilon \rightarrow 0, s \geq 0$ and $S \geq 0$.

Indeed, first estimate of (4.71) is an immediate corollary of (4.63) and the scaling invariance of the $L^{\infty}$-norm. Analogously, the second estimate of (4.71) follows from (4.64) and the scaling invariance of the $L^{1}$-norm of the time derivative. Finally, the third one is just repeats estimate (4.70).

We are now ready to pass to the limit $\varepsilon \rightarrow 0$ and formulate the main result of this section which is the analog of Theorem 2.17 for the quasi-linear case.

Theorem 4.16. Let the assumptions of Section 4.1 hold and let $\left(u_{\varepsilon}(s), t_{\varepsilon}(s)\right), \varepsilon \rightarrow$ 0 be solutions of problem (4.68) constructed above. Then, there exists a sequence 
$\varepsilon_{n} \rightarrow 0$ such that the associated solutions $\left(u_{n}(s), t_{n}(s)\right):=\left(u_{\varepsilon_{n}}(s), t_{\varepsilon_{n}}(s)\right)$ tend to the limit pair $(u(s), t(s)),\left\|\partial_{s} u(s)\right\|_{L^{2}} \leq 1$, in the following sense:

$$
\begin{aligned}
& u_{n} \rightarrow u \text { weakly* in } L^{\infty}\left([0, S], W^{1, p}(\Omega)\right) \cap W^{1, \infty}\left([0, S], L^{2}(\Omega)\right), \\
& u_{n} \rightarrow u \text { strongly in } L^{p}\left([0, S], H^{1}(\Omega)\right), \\
& \Theta_{u_{n}} \rightarrow \Theta_{u} \text { weakly* in } L^{\infty}\left([0, S], L^{2}(\Omega)\right), \\
& \partial_{s} u_{n} \rightarrow \partial_{s} u \text { weakly in } L^{1 / \kappa}\left([0, S], W^{\kappa, 2}(\Omega)\right), \quad 0 \leq \kappa<1, \\
& t_{n} \rightarrow t \text { weakly* in } W^{1, \infty}([0, S]), \\
& \int_{S_{2}}^{S_{1}}\left(\Psi\left(\partial_{s} u_{n}(s)\right), 1\right)_{L^{2}} \mathrm{~d} s \rightarrow \int_{S_{2}}^{S_{1}}\left(\Psi\left(\partial_{s} u(s)\right), 1\right)_{L^{2}} \mathrm{~d} s, \quad S_{1} \geq S_{2} \geq 0, \\
& \text { e) } \int_{S_{1}}^{S_{2}}\left(\partial C_{\varepsilon_{n}}\left(\partial_{s} u_{n}(s), \partial_{s} u_{n}(s)\right)_{L^{2}} \mathrm{~d} s \rightarrow \int_{S_{1}}^{S_{2}}\left(\varphi_{0}(s), \partial_{s} u(s)\right)_{L^{2}} \mathrm{~d} s,\right.
\end{aligned}
$$

for a function $\varphi_{0} \in L^{\infty}\left([0, S], L^{2}(\Omega)\right)$ with $\varphi_{0}(s) \in \partial \mathcal{C}_{0}\left(\partial_{s} u(s)\right)$ for almost all $s$. The limit functions $(t(s), u(s))$ satisfy the following weakened version of (4.69):

$$
\left\{\begin{array}{l}
\partial \Psi\left(\partial_{s} u(s)\right)+\partial \mathcal{C}_{0}\left(\partial_{s} u(s)\right) \ni \operatorname{div}\left(b\left(\nabla_{x} u(s)\right)\right)-f(t(s), u(s)), \\
t^{\prime}(s)+\left\|\partial_{s} u(s)\right\|_{L^{2}}=R(s), \quad t(0)=0 \\
u(0)=u_{0}
\end{array}\right.
$$

where the function $R \in L^{\infty}(\Omega), 0 \leq R(s) \leq 1$ satisfies the following property:

$$
\int_{S_{2}}^{S_{1}} R(s) \mathrm{d} s \geq C \min \left\{S_{1}-S_{2},\left(S_{1}-S_{2}\right)^{K}\right\}, \quad S_{1} \geq S_{2} \geq 0,
$$

where the positive constants $C$ and $K$ are independent of $S_{1}$ and $S_{2}$. Finally, the scaled time $t(s)$ satisfies

$$
s \geq t(s) \geq \beta s-C, \quad 1 \geq t^{\prime}(s) \geq 0 .
$$

Proof. Indeed, the weak convergences (4.72a), (4.72c), and (4.72e) are immediate corollaries of the boundedness of $u_{\varepsilon}, t_{\varepsilon}$ and $\Theta_{u_{\varepsilon}}$ in the corresponding functional spaces, see estimates (4.71). In order to obtain (4.72d), it is sufficient to note that, due to (4.71), the sequence $\partial_{s} u_{\varepsilon}$ is uniformly bounded in $L^{\infty}\left([0, S], L^{2}(\Omega)\right) \cap$ $L^{1}\left([0, S], H^{1}(\Omega)\right)$ and to use the standard interpolation embedding

$$
L^{1 / \kappa}\left([0, S], W^{\kappa, 2}(\Omega)\right) \subset L^{\infty}\left([0, S], L^{2}(\Omega)\right) \cap L^{1}\left([0, S], H^{1}(\Omega)\right) .
$$

Moreover, estimates (4.75) follow from the fact that

$$
t_{n} \rightarrow t \text { strongly in } C^{\alpha}([0, S]), \quad 0<\alpha<1 .
$$


In order to verify the remaining properties, we first prove that

$$
\Theta_{u_{n}} \rightarrow \Theta_{u} \text { strongly in } L^{p}\left([0, S], W^{-\kappa, 2}(\Omega)\right), \kappa>0
$$

and that

$$
\Theta_{u}(s)=\operatorname{div}\left(b\left(\nabla_{x} u(s)\right)-f(t(s), u(s)) .\right.
$$

Indeed, since the uniform boundedness of $\Theta_{u_{n}}$ in $L^{\infty}\left([0, S], L^{2}(\Omega)\right)$ is already known, for proving (4.77), it is sufficient to obtain some information about the smoothness of $s \mapsto \Theta_{u_{n}}(s)$. Moreover, since the operator $f(t, u)$ is subordinated to $\operatorname{div}\left(b\left(\nabla_{x} u\right)\right)$ and (4.76) holds, it is sufficient to consider only the most complicated term $\operatorname{div}\left(b\left(\nabla_{x} u_{n}(s)\right)\right.$. In particular, (4.77) will be proved if we prove that

$$
b\left(\nabla_{x} u_{n}\right) \in W^{1 /(p-1), 1}\left([0, S], L^{1}(\Omega)\right)
$$

and uniformly bounded in that space. To this end we note that assumptions (4.3) imply

$$
\left|b\left(w_{1}\right)-b\left(w_{2}\right)\right| \leq C\left(\left\|w_{1}\right\|+\left\|w_{2}\right\|\right)^{p-1-\alpha}\left|w_{1}-w_{2}\right|^{\alpha}
$$

for all $0 \leq \alpha \leq 1$. Fixing $\alpha=1 /(p-1)$ and using the Hölder inequality, we infer

$$
\begin{aligned}
& \left\|b\left(w_{1}\right)-b\left(w_{2}\right)\right\|_{L^{1}([0,1] \times \Omega)} \\
& \leq C\left(\left\|w_{1}\right\|_{L^{p}([0,1] \times \Omega)}+\left\|w_{2}\right\|_{L^{p}([0,1] \times \Omega)}\right)^{p-1-\alpha}\left\|w_{1}-w_{2}\right\|_{L^{1}([0,1] \times \Omega)}^{1 /(p-1)}
\end{aligned}
$$

for every $w_{1}, w_{2} \in L^{p}([0,1] \times \Omega)$. Using now this formula with $w_{1}:=\nabla_{x} u_{n}(\cdot+$ $\left.s_{1}\right), w_{2}:=\nabla_{x} u_{n}\left(\cdot+s_{2}\right)$ and using $(4.71)$, we get

$$
\begin{aligned}
& \left\|b\left(\nabla_{x} u_{n}\left(\cdot+s_{1}\right)\right)-b\left(\nabla_{x} u_{n}\left(\cdot+s_{2}\right)\right)\right\|_{L^{1}([0,1] \times \Omega)} \\
& \quad \leq C\left\|\nabla_{x} u_{1}\left(\cdot+s_{1}\right)-\nabla_{x} u_{1}\left(\cdot+s_{2}\right)\right\|_{L^{1}([0,1] \times \Omega)}^{1 /(p-1)} \\
& \quad \leq C\left|s_{1}-s_{2}\right|^{1 /(p-1)}\left(\int_{s_{1}}^{s_{2}+1}\left\|\partial_{s} u_{n}(s)\right\|_{H^{1}} \mathrm{~d} s\right)^{1 /(p-1)} .
\end{aligned}
$$

Thus, the uniform boundedness of $b\left(\nabla_{x} u_{n}\right)$ in the space (4.79) is verified and, consequently, convergence (4.77) is also verified. Moreover, this strong convergence together with the fact that $f(t, u)$ is subordinated to the leading monotone part $\operatorname{div}\left(b\left(\nabla_{x} u\right)\right)$ allows us to pass to the limit $n \rightarrow \infty$ in the elliptic problem

$$
\Theta_{u_{n}}(s)=\operatorname{div} b\left(\nabla_{x} u_{n}(s)\right)-f\left(t_{n}(s), u_{n}(s)\right)
$$

and establish the limit equality (4.78) (using the standard monotone-operator arguments). Moreover, since $\Theta_{u_{n}} \rightarrow \Theta_{u}$ strongly in $L^{p}\left([0, S], H^{-1}(\Omega)\right)$, estimate (4.3)(3) implies in a standard way that $u_{n} \rightarrow u$ strongly in $L^{p}\left([0, S], H^{1}(\Omega)\right.$ ). Thus, convergence (4.72b) is also verified. Moreover, using (4.72d) we see that, for every $S>0$

$$
\lim _{n \rightarrow \infty}\left\langle\Theta_{u_{n}}, \partial_{s} u_{n}\right\rangle_{S}=\left\langle\Theta_{u}, \partial_{s} u\right\rangle_{S},
$$

where $\langle\cdot \cdot\rangle_{S}$ denotes the scalar product in $L^{2}([0, S] \times \Omega)$. 
We are now ready to prove (4.72f) and pass to the limit $n \rightarrow \infty$ in equations (4.68). To this end,we fix $\theta_{n} \in L^{\infty}\left(\mathbb{R}_{+} \times \Omega\right)$ and $\varphi_{n} \in L^{\infty}\left(\mathbb{R}_{+}, L^{2}(\Omega)\right)$ such that

$$
\theta_{n}+\varphi_{n}=\Theta_{u_{n}}, \quad \theta_{n}(s) \in \partial \Psi\left(\partial_{s} u_{n}(s)\right), \quad \varphi_{n}(s) \in \partial C_{\varepsilon_{n}}\left(\partial_{s} u_{n}(s)\right) .
$$

Such decomposition exists since $u_{n}(s)$ satisfies the first equation of (4.68). Moreover, since $\left\|\theta_{n}\right\|_{L^{\infty}} \leq C$ then the above sequences are uniformly bounded in these spaces. So, without loss of generality, we may assume that

$$
\begin{aligned}
& \theta_{n} \rightarrow \theta_{0} \text { weakly* in } L^{\infty}([0, S] \times \Omega), \\
& \varphi_{n} \rightarrow \varphi \text { weakly* in } L^{\infty}\left([0, S], L^{2}(\Omega)\right)
\end{aligned}
$$

for every $S \in \mathbb{R}_{+}$and

$$
\theta_{0}+\varphi_{0}=\Theta_{u}
$$

Thus, in order to verify that $(u, t)$ solves the first equation of (4.73), we only need to prove that

$$
\theta_{0} \in \partial \Psi\left(\partial_{s} u\right), \quad \varphi_{0} \in \partial \mathcal{C}_{0}\left(\partial_{s} u\right) .
$$

To this end, we first recall that the monotonicity of operators $\partial \Psi(v)$ and $\partial \mathcal{C}_{\varepsilon_{n}}(v)$ together with the strong convergence (in $V=L^{2}([0, S] \times \Omega)$ ) of the operator $\partial \mathcal{C}_{\varepsilon_{n}}$ to $\partial \mathcal{C}_{0}$ established in Lemma A.4, give

$$
\liminf _{n \rightarrow \infty}\left\langle\theta_{n}, \partial_{s} u_{n}\right\rangle_{S} \geq\left\langle\theta_{0}, \partial_{s} u\right\rangle_{S}, \liminf _{n \rightarrow \infty}\left\langle\varphi_{n}, \partial_{s} u_{n}\right\rangle_{S} \geq\left\langle\varphi_{0}, \partial_{s} u\right\rangle_{S}
$$

for every $S \geq 0$. Indeed, let us verify the more complicated second estimate (leaving the analogous, but simpler first one to the reader). Let $\left(\partial_{s} u, w_{0}\right)$ be an arbitrary point of the graph of $\partial \mathcal{C}_{0}$ (which exists since $\left.\left\|\partial_{s} u(s)\right\|_{L^{2}} \leq 1\right)$. Then, Lemma A.4 with $V=L^{2}\left([0, S] \times L^{2}(\Omega)\right)$ gives the existence of a sequence $\left(v_{n}, w_{n}\right)$ converging strongly in $L^{2}([0, S] \times \Omega)^{2}$ to the pair $\left(\partial_{s} u, w_{0}\right)$ and such that

$$
w_{n} \in \partial \mathcal{C}_{\varepsilon_{n}}\left(v_{n}\right)
$$

Then, due to the monotonicity of $\partial \mathcal{C}_{\varepsilon_{n}}$, we have

$$
\begin{aligned}
\left\langle\varphi_{n}, \partial_{s} u_{n}\right\rangle_{S} & =\left\langle\varphi_{n}-w_{n}, \partial_{s} u_{n}-v_{n}\right\rangle_{S}+\left\langle w_{n}, \partial_{s} u_{n}-v_{n}\right\rangle_{S}+\left\langle\varphi_{n}, v_{n}\right\rangle_{S} \\
& \geq\left\langle w_{n}, \partial_{s} u_{n}-v_{n}\right\rangle_{S}+\left\langle\varphi_{n}, v_{n}\right\rangle_{S} .
\end{aligned}
$$

Passing to the limit $n \rightarrow \infty$ in this estimate, we obtain the second estimate of (4.87). Thus, estimates (4.87) are verified.

On the other hand, due to the convergence (4.82), we have

$$
\lim _{n \rightarrow \infty}\left(\left\langle\varphi_{n}, \partial_{s} u_{n}\right\rangle_{S}+\left\langle\varphi_{n}, \partial_{s} u_{n}\right\rangle_{S}\right)=\left\langle\varphi_{0}, \partial_{s} u\right\rangle_{S}+\left\langle\varphi_{0}, \partial_{s} u\right\rangle_{S}
$$

Inequalities (4.87) together with the equality (4.90) imply that we have exact equalities in (4.87), i.e.

$$
\lim _{n \rightarrow \infty}\left\langle\theta_{n}, \partial_{s} u_{n}\right\rangle_{S}=\left\langle\theta_{0}, \partial_{s} u\right\rangle_{S}, \lim _{n \rightarrow \infty}\left\langle\varphi_{n}, \partial_{s} u_{n}\right\rangle_{S}=\left\langle\varphi_{0}, \partial_{s} u\right\rangle_{S}
$$


Since the operators $\partial \Psi(v)$ and $\partial \mathcal{C}_{0}(v)$ are maximal monotone, the estimates (4.87) imply in a standard way the inclusions (4.86) and, consequently, the limit functions $(u(s), t(s))$ satisfy the first equation of (4.73).

Furthermore, using the identity $(\partial \Psi(v), v)=\Psi(v)$, we transform the first equality of (4.91) to

$$
\lim _{n \rightarrow \infty} \int_{0}^{S}\left(\Psi\left(\partial_{s} u_{n}(s)\right), 1\right)_{L^{2}} \mathrm{~d} s=\int_{0}^{S}\left(\Psi\left(\partial_{s} u(s)\right), 1\right)_{L^{2}} \mathrm{~d} s
$$

which holds for every $S>0$. Subtracting equations (4.92) for $S=S_{1}$ and $S=S_{2}$, we obtain convergence (4.72f). The convergence (4.72e) follows analogously from the second equality of (4.91). Thus, all of the convergences (4.72) are verified.

Let us now consider the second equation of (4.68) and let

$$
R(s):=t^{\prime}(s)+\left\|\partial_{s} u(s)\right\|_{L^{2}} .
$$

Then, the second equation of (4.73) are satisfied by definition and, passing to the limit in the second equation of (4.68) and using that $\partial_{s} u_{n}$ converges weakly in $L^{2}$ to the limit function $\partial_{s} u$, we see that

$$
0 \leq R(s) \leq 1
$$

Unfortunately, we do not have the strong convergence of $\partial_{s} u_{n}$ to $\partial_{S} u$ in $L^{2}([0, S] \times$ $\Omega$ ) in order to conclude that $R(s) \equiv 1$.

Finally, to finish the proof of the theorem, we only need to verify estimate (4.74). But it is factually done in the proof of Corollary 2.18. Thus, Theorem 4.16 is proved.

We conclude this section by proving that we can fix $K=1$ in (4.74) and, consequently, the scaling factor $R(s)$ is separated from zero (exactly as in the semilinear case).

Theorem 4.17. Let the assumptions of Theorem 4.16 hold. Then, the scaling factor $R(s)$ from the second equation (4.73) satisfies

$$
1 \geq R(s) \geq \beta>0 \quad \text { for a.a. } s \in[0, S] .
$$

Proof. Analyzing the proof of Theorem 2.21, we conclude that we can repeat it word by word for the quasi-linear case as well if the higher energy estimate (2.90) is a priori known. Thus, we only need to verify this estimate. However, in contrast to the semi-linear case, we do not have the uniqueness and, by this reason, the proof indicated in Lemma 2.22 fails here. Indeed, although we are still able to construct directly a strong solution $(u(s), t(s))$ of (4.68) satisfying (2.90) by the above approximation scheme, it is not obvious that the corresponding solution $u(\tau)$ of (4.13) will satisfy the energy estimate (4.18) involved in our definition of a strong solution. Therefore, in order to overcome this difficulty, we need to deduce (2.90) directly from (4.18). 
We first recall that the higher energy estimate (4.18) can be formulated in the initial time scale $t$ as follows:

$$
\begin{aligned}
& \varepsilon\left\|\partial_{t} u\left(t_{2}\right)\right\|_{L^{2}}^{2}-\varepsilon\left\|\partial_{t} u\left(t_{1}\right)\right\|_{L^{2}}^{2}+2 \alpha \int_{t_{1}}^{t_{2}}\left\|\nabla_{x} \partial_{\tau} u(t)\right\|_{L^{2}}^{2} \mathrm{~d} t \\
& +2 \int_{t_{1}}^{t_{2}}\left[\left(f_{t}^{\prime}(t, u(t)), \partial_{t} u(t)\right)_{L^{2}}+\left(f_{u}^{\prime}(t, u(t)) \partial_{t} u(t), \partial_{t} u(t)\right)_{L^{2}}\right] \mathrm{d} t \leq 0
\end{aligned}
$$

for almost all $t_{1}, t_{2} \geq 0, t_{2} \geq t_{1}$.

It is not difficult to see that, in order to deduce (2.90) from (4.95), it is sufficient to be able to apply (B.2) with

$$
\phi(t):=\frac{1}{1+\left\|\partial_{t} u(t)\right\|_{L^{2}}}
$$

to estimate (4.95) (see formulae (2.66) and (2.69)). However, we are not able to apply Lemma B.1 here, since the function $\phi$ is only $L^{\infty}$, but does not belong to $C^{1}$. In order to overcome this obstacle, we use Lemma B. 2 and the convexity arguments instead. Indeed,

$$
\left\|\partial_{t} u(t)\right\|_{L^{2}}^{2}=F(\phi(t)), \text { where } F(z):=\left(\frac{1}{z}-1\right)^{2}
$$

and the function $F$ is decreasing on the interval $(0,1]$ (since $\phi(t) \in(0,1]$ for all $t$, we need the monotonicity on that interval only). Thus, Lemma B.2 is applicable to (4.95) and the obtained estimate (B.3) is equivalent to the desired estimate (2.90).

This finishes the proof of the theorem.

Finally, exactly as in the semilinear case, we can rescale the independent variable to obtain a strict arclength parametrization, see Remark 2.19. Therefore, the proved Theorem 4.16 provide a pair $(\bar{t}, \bar{u})$ with

$$
\bar{t} \in W^{1, \infty}\left(\mathbb{R}_{+}\right), \quad \bar{u} \in W^{1, \infty}\left(\mathbb{R}_{+}, L^{2}(\Omega)\right) \cap L^{\infty}\left(\mathbb{R}_{+}, W_{0}^{1, p}(\Omega)\right)
$$

satisfying the analog of (4.69), namely

$$
\left\{\begin{array}{l}
\partial \Psi\left(\partial_{\bar{s}} \bar{u}(\bar{s})\right)+\partial \mathcal{C}_{0}\left(\partial_{\bar{s}} \bar{u}(\bar{s})\right) \ni \operatorname{div}\left(b\left(\nabla_{x} \bar{u}(\bar{s})\right)\right)-f(\bar{t}(\bar{s}), \bar{u}(\bar{s})), \\
\bar{t}^{\prime}(\bar{s})+\left\|\partial_{\bar{s}} \bar{u}(\bar{s})\right\|_{L^{2}}=1, \quad \bar{t}(0)=0 \\
u(0)=u_{0}
\end{array}\right.
$$

\section{An application in magnetostriction}

We consider a bounded Lipschitz domain $\Omega \subset \mathbb{R}^{d}$ for $d \in\{2,3\}$ together with a Dirichlet part $\Gamma_{\text {Dir }} \subset \partial \Omega$ having positive surface measure. For a fixed time the 
state of the body is described by the displacement $y: \Omega \rightarrow \mathbb{R}^{d}$ with $y \in Y:=$ $H_{\Gamma_{\text {Dir }}}^{1}(\Omega)=\left\{y \in H^{1}(\Omega),\left.y\right|_{\Gamma_{\text {Dir }}}=0\right\}$ and the magnetization $m: \Omega \rightarrow \mathbb{R}$ satisfying $m \in H^{1}(\Omega)$. Often it is assumed $|m(x)|=M_{\text {sat }}$ but in our model this will be only satisfied approximately through a penalty term in the energy functional.

To put the system into the abstract form (3.1) it suffices to explain the form of the Hilbert spaces $H$, the dissipation potential $\Psi: H \rightarrow[0, \infty)$, main operator $\mathcal{B}$, and the potential $\Phi(t, \cdot)$. The magnetization $m$ plays the role of our former variable $u$. Hence, we let

$$
H=H^{1}(\Omega)^{d} \quad \text { and } \quad \widetilde{\Psi}(m)=\int_{\Omega} \rho(x)|m(x)| \mathrm{d} x,
$$

where $\rho \in L^{\infty}(\Omega)$ satisfies $\rho(x) \geq \rho_{0}>0$ a.e. in $\Omega$.

We first define an energy functional in terms of the displacement $y$ and $m$, namely

$$
\widetilde{\mathcal{E}}(t, y, m)=\int_{\Omega} \frac{\alpha}{2}|\nabla m|^{2}+W(x, e(y), m) \mathrm{d} x-\left\langle\ell_{\text {mech }}(t), y\right\rangle-\left\langle H_{\text {ext }}(t), m\right\rangle,
$$

where $\ell_{\text {mech }} \in C^{1}\left([0, T], Y^{*}\right)$ is the mechanical loading via volume and surface forces, whereas $H_{\mathrm{ext}} \in C^{1}\left([0, T], H^{*}\right)$ denotes the external magnetic field.

The stored energy density $W$ may depend on the material point $x \in \Omega$, but for notational simplicity we suppress this dependence. More importantly, $W$ depends on the linearized strain tensor $e(y)=\frac{1}{2}\left(\nabla y+(\nabla y)^{T}\right) \in \mathbb{R}_{\text {sym }}^{d \times d}$ and the magnetization vector $m$ in the form

$$
W(e, m)=\frac{1}{2}(e-E(m)): \mathbb{C}:(e-E(m))+W_{\text {aniso }}(m)+\frac{1}{4 \delta}\left(|m|^{2}-M_{\mathrm{sat}}^{2}\right)^{2}
$$

where $E \in C\left(\mathbb{R}^{d} ; \mathbb{R}_{\text {sym }}^{d \times d}\right)$ is the magnetization strain, which we assume to be linear in the form $E(m)=\sum_{j=1}^{d} m_{j} E_{j}$ for $m=\left(m_{1}, \ldots, m_{d}\right)$, and $\mathbb{C}$ is the elastic forthorder tensor, which is assumed to satisfy $e: \mathbb{C}: e=\sum_{i, j, k, l=1}^{d} e_{i j} C_{i j k l} e_{k l} \geq c_{0}|e|^{2}$. The term $W_{\text {aniso }} \in C^{2}\left(\mathbb{R}^{d}\right)$ satisfies $W_{\text {aniso }}(m) \geq 0,\left|W_{\text {aniso }}^{\prime \prime}(m)\right| \leq C$, and contains the anisotropy information, e.g. about easy axes, while the last term penalized $m$ to have a modulus close to the saturation value.

In the quasistatic setting we may assume that the displacement is always in equilibrium, i.e. $\widetilde{\mathcal{E}}_{y}^{\prime}(t, y(t), m(t))=0$ for all $[0, T]$. From the quadratic nature of W in terms of $e(y)-E(m)$ we see that $y$ can be found by a linear elliptic PDE giving $y(t)=\mathbf{L}(m(t), \ell(t))$, where $\mathbf{L}: L^{2}(\Omega) \times H^{*} \rightarrow H$ is a bounded linear operator. To prove this result one uses the Lax-Milgram lemma and the Korn inequality $\int_{\Omega} e(\hat{y}): \mathbb{C}: e(\hat{y}) \mathrm{d} x \geq c_{1}\|\hat{y}\|_{H^{1}}^{2}$ for all $\hat{y} \in Y$.

Thus, we are able to define

$$
\mathcal{E}(t, m)=\widetilde{\mathcal{E}}(t, \mathbf{L}(m, \ell(t)), m)=\min \{\widetilde{\mathcal{E}}(t, \hat{y}, m), \hat{y} \in Y\} .
$$


Defining $\mathcal{B}: H \rightarrow H^{*}$ via $\mathcal{B} m=-\alpha \Delta m+\alpha m$ we see that the potential $\Phi(t, m)=$ $\mathcal{E}(t, m)-\frac{1}{2}\langle\mathcal{B} m, m\rangle$ takes the form

$$
\Phi(t, m)=\int_{\Omega} W(e(\mathbf{L}(m, \ell(t)), m))-\frac{\alpha}{2}|m|^{2} \mathrm{~d} x-\langle\ell(t), \mathbf{L}(m, \ell(t))\rangle-\left\langle H_{\mathrm{ext}}(t), m\right\rangle .
$$

It is not difficult to check that $\Phi$ satisfies all the assumptions of Section 3. Thus, the abstract theory developed there applies in the present case.

\section{A. Approximation of convex functions and their subdifferentials}

The aim of this appendix is to study the approximations of several convex functionals and the associated subdifferentials which associated with problem (2.1). We start by constructing the approximations of a convex order one homogeneous functional on a finite-dimensional space by smooth functionals.

To be precise, let a function $\Psi: \mathbb{R}^{m} \rightarrow \mathbb{R}_{+}$satisfy assumptions (2.3) Then, obviously, the function $\Psi(v)$ should be Lipschitz continuous with respect to $v$. Indeed, according to (2.3),

$$
|\Psi(v+w)-\Psi(v)| \leq \Psi(w) \leq \kappa_{2}\|w\| .
$$

Let us fix now a non-negative cut-off function $\varphi \in C_{0}^{\infty}\left(\mathbb{R}^{m}\right)$ such that (i) $\operatorname{supp} \varphi \subset$ $B_{0}^{1}:=\left\{v \in \mathbb{R}^{m},\|v\|<1\right\}, \quad$ (ii) $\int_{\mathbb{R}^{m}} \varphi(v) \mathrm{d} v=1$. and introduce the standard smoothing kernel $\varphi_{\delta}(v):=\frac{1}{\delta^{m}} \varphi\left(\frac{v}{\delta}\right), \delta>0$. Then, the smooth approximations $\Psi_{\delta}(v)$ can be defined as follows:

$$
\Psi_{\delta}(v)=\int_{\mathbb{R}^{m}} \varphi_{\delta}(s) \Psi(v-s) \mathrm{d} s .
$$

The next lemma indicates the obvious properties of functions $\Psi_{\delta}$.

Lemma A.1. Let the above assumptions hold. Then, for every $\delta>0$ the functions $\Psi_{\delta}(v)$ are smooth, convex and satisfy the first estimate of (2.3) with the same constants $\kappa_{i}$. Moreover, the second assumption of (2.3) now reads

$$
\Psi_{\delta}(\alpha v)=|\alpha| \Psi_{\delta /|\alpha|}(v), \quad \alpha \in \mathbb{R}, \quad v \in \mathbb{R}^{m} .
$$

Furthermore, $\Psi_{\delta}$ converges to $\Psi$ as $\delta \rightarrow 0$ and

$$
\left|\Psi_{\delta}(v)-\Psi(v)\right| \leq \kappa_{2} \delta, \quad v \in \mathbb{R}^{m} .
$$

Indeed, all of the assertions of the lemma are standard corollaries of (2.3) and (A.1)(A.2). We recall that smooth homogeneous order $\mathrm{p}$ functions satisfy the Euler identity $f^{\prime}(v) \cdot v=p f(v)$. The next lemma gives the analog of this identity for the functions $\Psi_{\delta}$. 
Lemma A.2. Let the above assumptions hold. Then

$$
\Psi_{\delta}^{\prime}(v) . v-\Psi_{\delta}(v)=P_{\delta}(v),
$$

where

$$
P_{\delta}(v):=\int_{\mathbb{R}^{m}} \psi_{\delta}(s) \Psi(v-s) \mathrm{d} s, \quad \psi_{\delta}(s):=\frac{m}{\delta^{m}} \varphi\left(\frac{s}{\delta}\right)-\frac{1}{\delta^{m+1}} \varphi^{\prime}\left(\frac{s}{\delta}\right) . s .
$$

In particular,

$$
\left|P_{\delta}(v)\right| \leq C \delta,
$$

where the constant $C$ is independent of $\delta$ and $v$.

Proof. Differentiating identity (A.3) with respect to $\alpha$ near $\alpha=1$, we get

$$
\Psi_{\delta}^{\prime}(v) . v-\Psi_{\delta}(v)=\left.\frac{d}{d \alpha} \Psi_{\delta / \alpha}(v)\right|_{\alpha=1}
$$

which, after simple calculations, gives (A.5) and (A.6). Thus, we only need to verify estimate (A.7). To this end, we note that

$$
\int_{\mathbb{R}^{m}} \psi_{\delta}(s) \mathrm{d} s=\int_{\mathbb{R}^{m}}\left[m \varphi(s)+\varphi^{\prime}(s) . s\right] \mathrm{d} s=0 .
$$

Indeed, since $\operatorname{div}(\varphi(s) \vec{s})=m \varphi(s)+\varphi^{\prime}(s) . s$, the Gauss integration by parts formula gives (A.8). Using that, we can rewrite $b_{\delta}(v)$ as $b_{\delta}(v)=\int_{\mathbb{R}^{m}} \psi_{\delta}(s)[\Psi(v-s)-$ $\Psi(v)] \mathrm{d} s$ and, consequently, due to (A.1),

$$
\left|P_{\delta}(v)\right| \leq \kappa_{2} \int_{\mathbb{R}^{m}}\left|\psi_{\delta}(s)\right| \cdot|s| \mathrm{d} s=\kappa_{2} \delta \int_{\mathbb{R}^{m}}|s| \cdot\left|m \varphi(s)-\varphi^{\prime}(s) . s\right| \mathrm{d} s=C \delta .
$$

Lemma A. 2 is proved.

Our next task is to study the convergence of subdifferentials of functionals $\Psi_{\delta}(v)$. To be more precise, we consider the following equation in $\mathbb{R}^{m}$ :

$$
\Psi_{\delta}^{\prime}(v)+\varepsilon v=f, \quad f \in \mathbb{R}^{n}, \varepsilon>0 .
$$

Lemma A.3. Let the above assumptions hold. Then, for every $f \in \mathbb{R}^{n}$ and every $\delta>0$, problem (A.9) has a unique solution $v_{\delta}$, these solutions converge as $\delta \rightarrow 0$ to the unique solution $v_{0}$ of the limit problem

$$
f \in \partial \Psi(v)+\varepsilon v
$$

and the following estimate holds:

$$
\left\|v_{\delta}-v_{0}\right\| \leq C(\delta / \varepsilon)^{1 / 2},
$$

where the constant $C$ is independent of $\delta, \varepsilon$ and $f$. 
Proof. Let

$$
A_{\delta}(v):=\Psi_{\delta}(v)+\varepsilon / 2\|v\|^{2} .
$$

Since $\Psi_{\delta}(v)+\varepsilon / 2\|v\|^{2}$ is strictly convex, then the minimum in of $A_{\delta}(v)$ over $v \in$ $\mathbb{R}^{m}$ exists and $v_{\delta}=\operatorname{Argmin}_{v \in \mathbb{R}^{m}}\left\{\Psi_{\delta}(v)+\varepsilon / 2\|v\|^{2}\right\}$ gives a unique solution of (A.9) and the analogous description holds for the solution $v_{0}$ of the limit problem (A.10). Moreover, due to estimate (A.4), we have $\left|A_{\delta}\left(v_{\delta}\right)-A_{0}\left(v_{0}\right)\right|=\left|\min A_{\delta}-\min A_{0}\right| \leq$ $C \delta$ and, consequently, $\left|A_{0}\left(v_{\delta}\right)-A_{0}\left(v_{0}\right)\right| \leq 2 C \delta$. Moreover, the strong convexity of $A_{0}$ gives $A_{0}\left(v_{\delta}\right) \geq A_{0}\left(v_{0}\right)+\varepsilon / 4\left\|v_{\delta}-v_{0}\right\|^{2}$. Together this implies (A.11) and the proof of the lemma is finished.

We conclude by studying the functionals on the infinite-dimensional phase spaces which arise in our study the rate independent limit $\varepsilon \rightarrow 0$. Namely, let

$$
V=V_{p, \kappa}:=L^{p}\left([0, T], W_{0}^{\kappa, 2}(\Omega), \quad V^{*}=L^{q}\left([0, T], W^{-\kappa, 2}(\Omega)\right)\right.
$$

for some $2 \leq p<\infty, 1 / p+1 / q=1$ and $0 \leq \kappa \leq 1$ and let the functionals $\mathcal{C}_{\varepsilon}$ and $\mathcal{C}_{0}$ on $V$ be defined as follows:

$$
\mathcal{C}_{\varepsilon}(v):=\int_{0}^{T} \mathcal{C}_{\varepsilon}(v(s)) \mathrm{d} s, \quad \mathcal{C}_{0}(v):=\int_{0}^{T} \mathcal{C}_{0}(v(s)) \mathrm{d} s,
$$

where the functions $\mathcal{C}_{\varepsilon}$ and $\mathcal{C}_{0}$ are defined by (2.70) and (2.73) respectively.

The next lemma shows that the subdifferentials $\partial \mathcal{C}_{\varepsilon}$ converge strongly as $\varepsilon \rightarrow$ 0 to the subdifferential $\partial \mathcal{C}_{0}$ of the limit functional $\mathcal{C}_{0}$ (with respect to the pairing $\left.\left(V, V^{*}\right)\right)$. This corresponds to Mosco convergence, see [1].

Lemma A.4. Let the above assumptions hold, let $\left(v_{0}, w_{0}\right) \in V \times V^{*}, v_{0} \in \operatorname{Dom}\left(\mathcal{C}_{0}\right)$, $w_{0} \in \partial \mathcal{C}_{0}\left(v_{0}\right)$ be arbitrary and let $\varepsilon_{n} \rightarrow 0$ be an arbitrary sequence of positive numbers. Then, there exist two sequences $v_{n} \in V$ and $w_{n} \in V^{*}$ such that

$$
v_{n} \in \operatorname{Dom}\left(\mathcal{C}_{\varepsilon_{n}}\right), \quad w_{n} \in \partial \mathcal{C}_{\varepsilon}\left(v_{n}\right)
$$

such that

$$
\left(v_{n}, w_{n}\right) \rightarrow\left(v_{0}, w_{0}\right) \text { strongly in } V \times V^{*} .
$$

Proof. Indeed, it follows in a standard way from the definition of a subdifferential that $\operatorname{Dom}\left(\mathcal{C}_{0}\right)=\left\{v_{0} \in V,\left\|v_{0}\right\|_{L^{2}} \leq 1\right\}$ and the set $\partial \mathcal{C}_{0}\left(v_{0}\right)$ has the following structure:

$$
\begin{aligned}
\partial \mathcal{C}_{0}\left(v_{0}\right)=\left\{\alpha(s) v_{0}(s), \quad \int_{0}^{T}|\alpha(s)|^{q}\left\|v_{0}(s)\right\|_{W^{-\kappa, 2}(\Omega)}^{q} \mathrm{~d} s<\infty,\right. \\
\alpha(s) \geq 0,\left(\alpha(s)=0 \text { if }\left\|v_{0}(s)\right\|_{\left.\left.L^{2}<1\right)\right\} .}\right.
\end{aligned}
$$


Thus, $w_{0}(s)=\alpha(s) v_{0}(s)$ for some non-negative function $\alpha$ satisfying the conditions formulated in (A.17). We now set

$$
v_{\varepsilon_{n}}(s):=\frac{\alpha(s)+\sqrt{\varepsilon_{n}}}{\alpha(s)+\varepsilon_{n}+\sqrt{\varepsilon_{n}}} v_{0}(s) .
$$

Then, obviously, $\left\|v_{\varepsilon_{n}}-v_{0}\right\|_{V} \leq \sqrt{\varepsilon_{n}}\left\|v_{0}\right\|_{V}$ and, consequently, $v_{\varepsilon_{n}} \rightarrow v_{0}$ strongly in $V$. Let us now check that $v_{n} \in \operatorname{Dom}\left(\mathcal{C}_{\varepsilon_{n}}\right)$. To this end, we first note that the function $\alpha$ must belong to $L^{1}([0, T])$. Indeed, for every $s$ such that $\left\|v_{0}(s)\right\|_{L^{2}}=1$, the interpolation estimate gives

$$
\left\|v_{0}(s)\right\|_{W^{-\kappa, 2}(\Omega)} \geq C\left\|v_{0}(s)\right\|_{W^{\kappa, 2}(\Omega)}^{-1}
$$

and, consequently,

$$
\begin{aligned}
\int_{0}^{T} \alpha(s) \mathrm{d} s & \leq\left\|\alpha v_{0}\right\|_{V^{*}}\left(\int_{\alpha(s) \neq 0} 1 /\left\|v_{0}(s)\right\|_{W^{-\kappa, 2}(\Omega)}^{p} \mathrm{~d} s\right)^{1 / p} \\
& \leq C\|\alpha v\|_{V^{*}}\left\|v_{0}\right\|_{V}<\infty .
\end{aligned}
$$

Moreover,

$$
\begin{aligned}
\frac{1}{1-\left\|v_{\varepsilon_{n}}(s)\right\|_{L^{2}}} & =\frac{\alpha(s)+\varepsilon_{n}+\sqrt{\varepsilon_{n}}}{\varepsilon_{n}+\left(\alpha(s)+\sqrt{\varepsilon_{n}}\right)\left(1-\left\|v_{0}(s)\right\|_{L^{2}}\right)} \\
& \leq 1+\frac{\alpha(s)+\sqrt{\varepsilon_{n}}}{\varepsilon_{n}}
\end{aligned}
$$

and, therefore,

$$
\mathcal{C}_{\varepsilon_{n}}\left(v_{\varepsilon_{n}}\right) \leq C+\int_{0}^{T} \varepsilon_{n} \ln \left(1+\frac{\alpha(s)+\sqrt{\varepsilon_{n}}}{\varepsilon_{n}}\right) \mathrm{d} s \leq C_{1}+\int_{0}^{T} \alpha(s) \mathrm{d} s<\infty .
$$

It only remains to note that, according to (A.20),

$$
\partial \mathcal{C}_{\varepsilon_{n}}\left(v_{\varepsilon_{n}}\right)(s)=\varepsilon_{n} \frac{w_{0}(s)+\sqrt{\varepsilon_{n}} v_{0}(s)}{\varepsilon_{n}+\left(\alpha(s)+\sqrt{\varepsilon_{n}}\right)\left(1-\left\|v_{0}(s)\right\|_{L^{2}}\right)}:=w_{n} .
$$

Thus,

$$
w_{n}(s)= \begin{cases}w_{0}(s)+\sqrt{\varepsilon_{n}} v_{0}(s), & \left\|v_{0}(s)\right\|_{L^{2}}=1, \\ \frac{\left(\varepsilon_{n}\right)^{3 / 2} v_{0}(s)}{\varepsilon_{n}+\sqrt{\varepsilon_{n}}\left(1-\left\|v_{0}(s)\right\|_{L^{2}}\right)}, & \left\|v_{0}(s)\right\|_{L^{2}}<1\end{cases}
$$

which, in turns, implies $\left\|w_{n}(s)-w_{0}(s)\right\|_{W^{-\kappa, 2}(\Omega)} \leq C \sqrt{\varepsilon_{n}}\left\|v_{0}(s)\right\|_{W^{-\kappa, 2}(\Omega)}$. Using $p \geq 2$ we have $w_{n} \in V^{*}$, and the last estimate implies $w_{n} \rightarrow w_{0}$ in $V^{*}$. Lemma A. 4 is proved. 


\section{B. A product rule for an integral estimate}

We consider solutions $\alpha \in L^{\infty}([0, T])$ of the integral equality

$$
\alpha(t)-\alpha(s) \leq \int_{s}^{t} g(\tau) \mathrm{d} \tau \quad \text { for all } t, s \in[0, T] \backslash N \text { with } s<t,
$$

where $g \in L^{1}([0, T])$ is given and $N \subset[0, T]$ has Lebesgue measure 0 . For differentiable $\alpha$ this estimate is equivalent to $\alpha^{\prime} \leq g$ a.e. on $[0, T]$. The desired product-rule estimate then means that for all nonnegative $\phi \in C^{1}([0, T])$ we have

$$
(\phi \alpha)^{\prime}=\phi \alpha^{\prime}+\phi^{\prime} \alpha \leq \phi g+\phi^{\prime} \alpha .
$$

The following result states that the corresponding integrated version holds without assuming that $\alpha$ is differentiable. The idea of the proof is to show that $\alpha$ has bounded variation and thus a distributional derivative $\mathrm{D} \alpha$ in the set of measures. Then, (B.1) implies that $g \mathrm{~d} t-\mathrm{D} \alpha$ is a nonnegative measure.

Lemma B.1. Let $g \in L^{1}([0, T])$ and $\phi \in C^{1}([0, T])$ with $\phi(t) \geq 0$ be given. If $\alpha \in L^{\infty}(\Omega)$ solves (B.1), then,

$$
\phi(t) \alpha(t)-\phi(s) \alpha(s) \leq \int_{s}^{t}\left[\phi(\tau) g(\tau)+\phi^{\prime}(\tau) \alpha(\tau)\right] d \tau \quad \text { for all } t, s \in[0, T] \backslash N .
$$

Proof. We first show that $\alpha$ lies in $\mathrm{BV}([0, T])$. For this define $\beta:[0, T] \rightarrow \mathbb{R}$ via $\beta(t)=\int_{0}^{t} g(r) \mathrm{d} r-\alpha(r)$. By (B.1) we conclude that $\beta$ is nondecreasing on $[0, T] \backslash N$. Thus, we may modify $\alpha$ on the null set $N$ in such a way that $\beta$ : $[0, T] \rightarrow \mathbb{R}$ is nondecreasing and hence of bounded variation.

Since we have $\alpha(t)=\int_{0}^{t} g(r) \mathrm{d} r-\beta(r)$ we conclude that $\alpha$ is of bounded variation as well. The distributional derivative $\mu:=\mathrm{D} \beta$ of $\beta$ is a positive measure, i.e., $\mu \in C_{0}^{0}([0, T])^{*}$ defined via $\int_{0}^{T} \psi(t) \mu(\mathrm{d} t)=-\int_{0}^{T} \psi^{\prime}(t) \beta(t) \mathrm{d} t$ for all $\psi \in$ $C_{c}^{\infty}([0, T])$. Clearly, we have $\mathrm{D} \alpha=g \mathrm{~d} t-\mu$. The chain rule $\mathrm{D}(\phi \alpha)=\phi \mathrm{D} \alpha+$ $\phi^{\prime} \alpha \mathrm{d} t$ is derived as follows:

$$
\begin{aligned}
\int_{0}^{T} \psi(t) \mathrm{D}(\phi \alpha)(\mathrm{d} t) & =-\int_{0}^{T} \psi^{\prime}(t) \phi(t) \alpha(t) \mathrm{d} t \\
& =-\int_{0}^{T}(\psi \phi)^{\prime} \alpha \mathrm{d} t+\int_{0}^{T} \psi \phi^{\prime} \alpha \mathrm{d} t \\
& =\int_{0}^{T} \psi \phi \mathrm{D} \alpha(\mathrm{d} t)+\int_{0}^{T} \psi \phi^{\prime} \alpha \mathrm{d} t .
\end{aligned}
$$

Now using the nonnegativity of $\phi$ and $\mu$ we obtain

$$
\mathrm{D}(\phi \alpha)=\phi(g \mathrm{~d} t-\mu)+\phi^{\prime} \alpha \mathrm{d} t \leq\left(\phi g+\phi^{\prime} \alpha\right) \mathrm{d} t .
$$

Thus, we conclude that $t \mapsto \int_{0}^{t}\left(\phi g+\phi^{\prime} \alpha\right) \mathrm{d} r-\phi(t) \alpha(t)$ is a nondecreasing function, and (B.2) is established. 
To conclude, we formulate one more useful analog of formula (B.2) for the case where the function $\phi$ is only $L^{\infty}$, but that $\alpha$ is related to $\phi$ via $\alpha=F \circ \phi$. For $\phi \in C^{1}$, the result is an immediate corollary of (B.2). The non-trivial part of the lemma is that this estimate remains true for the non-smooth $\phi$ as well.

The result was used in the proof of Theorem 4.17 only. There $F$ has the form $F(z)=(1 / z-1)^{2}$ for $z \in(0,1)$.

Lemma B.2. Let $\phi \in L^{\infty}([0, T]), g \in L^{1}([0, T])$ and $F \in C^{2}\left(\mathbb{R}^{2}\right)$ be three functions such that $\phi$ is nonnegative, $F$ is monotone (decreasing or increasing) and the functions $\alpha=F \circ \phi$ and $g$ satisfy estimate (B.1) for almost all $t, s \in[0, T]$. Then, for all $s, t \in[0, T] \backslash N$ with $s \leq t$ we have

$$
[\phi(t) \alpha(t)-\Phi(\phi(t))]-[\phi(s) \alpha(s)-\Phi(\phi(s))] \leq \int_{s}^{t} \phi(\tau) g(\tau) \mathrm{d} \tau,
$$

where $\Phi(z):=\int_{0}^{z} F(y) \mathrm{d} y$.

Proof. Let $\Pi=\left\{s=t_{0}<t_{1}<\cdots<t_{l-1}<t_{l}=t\right\}$ be an arbitrary partition of $[s, t]$ such that $t_{j} \in[0, T] \backslash N$ and let $F$ be increasing. Then $\Phi$ is convex and, analogously to (4.22), we infer that $F\left(\phi\left(t_{j-1}\right)\right)\left[\phi\left(t_{j}\right)-\phi\left(t_{j-1}\right)\right] \leq\left[\Phi\left(\phi\left(t_{j}\right)\right)-\right.$ $\left.\Phi\left(\phi\left(t_{j-1}\right)\right)\right]$. Thus,

$$
\begin{aligned}
& {[\phi(t) \alpha(t)-\Phi(\phi(t))]-[\phi(s) \alpha(s)-\Phi(\phi(s))]} \\
& =\sum_{j=1}^{l}\left[\phi\left(t_{j}\right) \alpha\left(t_{j}\right)-\Phi\left(\phi\left(t_{j}\right)\right)\right]-\left[\phi\left(t_{j-1}\right) \alpha\left(t_{j-1}\right)-\Phi\left(\phi\left(t_{j-1}\right)\right)\right] \\
& \leq \sum_{j=1}^{l} \phi\left(t_{j}\right) \alpha\left(t_{j}\right)-\phi\left(t_{j-1}\right) \alpha\left(t_{j-1}\right)-\alpha\left(t_{j-1}\right)\left(\phi\left(t_{j}\right)-\phi\left(t_{j-1}\right)\right) \\
& =\sum_{j=1}^{l} \phi\left(t_{j}\right)\left(\alpha\left(t_{j}\right)-\alpha\left(t_{j-1}\right) \leq \sum_{j} \phi\left(t_{j}\right) \int_{t_{j-1}}^{t_{j}} g(r) \mathrm{d} r=\int_{s}^{t} \phi_{\text {right }}^{\Pi}(r) g(r) \mathrm{d} r,\right.
\end{aligned}
$$

where $\phi_{\text {right }}^{\Pi}$ is the right-continuous piecewise interpolant of $\phi$ with respect to the partition $\Pi$. Using a suitable sequence of partitions ( $c f$. [9]), the right-hand side approaches the limit $\int_{s}^{t} \phi g \mathrm{~d} r$ and the result is established the case of increasing $F$.

Assume now that $F$ is decreasing. Then, $\Phi$ is concave and, analogously to (4.23), we have $F\left(\phi\left(t_{j}\right)\right)\left[\phi\left(t_{j}\right)-\phi\left(t_{j-1}\right)\right] \leq\left[\Phi\left(\phi\left(t_{j}\right)\right)-\Phi\left(\phi\left(t_{j-1}\right)\right)\right]$. Thus,

$$
\begin{aligned}
& {[\phi(t) \alpha(t)-\Phi(\phi(t))]-[\phi(s) \alpha(s)-\Phi(\phi(s))]} \\
& \leq \sum_{j=1}^{l} \phi\left(t_{j}\right) \alpha\left(t_{j}\right)-\phi\left(t_{j-1}\right) \alpha\left(t_{j-1}\right)-\alpha\left(t_{j}\right)\left(\phi\left(t_{j}\right)-\phi\left(t_{j-1}\right)\right) \\
& =\sum_{j=1}^{l} \phi\left(t_{j-1}\right)\left(\alpha\left(t_{j}\right)-\alpha\left(t_{j-1}\right) \leq \sum_{j} \phi\left(t_{j}\right) \int_{t_{j-1}}^{t_{j}} g(r) \mathrm{d} r=\int_{s}^{t} \phi_{l e f t}^{\Pi}(r) g(r) \mathrm{d} r,\right.
\end{aligned}
$$


where $\phi_{\text {left }}^{\Pi}$ is the left-continuous piecewise interpolant of $\phi$ with respect to the partition $\Pi$. Using a suitable sequence of partitions ( $c f$. [9]), the right-hand side approaches the limit $\int_{s}^{t} \phi g \mathrm{~d} r$ and the result is established for the case of decreasing $F$ as well.

\section{References}

[1] H. Atтouch, "Variational Convergence of Functions and Operators", Pitman Advanced Publishing Program, Pitman, 1984.

[2] A. V. BABIN and M. I. VISHIK, "Attraktory Evolyutsionnykh Uravnenii”, Nauka, Moscow, 1989.

[3] V. BELleRI and V. PATA, Attractors for semilinear strongly damped wave equations on $\mathbb{R}^{3}$, Discrete Contin. Dynam. Systems 7 (2001), 719-735.

[4] J.-F. BABADJIAN, G. A. FrANCFORT and M. G. MORA, Quasistatic evolution in nonassociative plasticity - the cap model, SIAM J. Math. Anal. 44 (2012), 245-292.

[5] F. CAGNETTI, A vanishing viscosity approach to fracture growth in a cohesive zone model with prescribed crack path, Math. Models Meth. Appl. Sci. 18 (2008), 1027-1071.

[6] P. Colli and A. Visintin, On a class of doubly nonlinear evolution equations, Comm. Partial Differential Equations 15 (1990), 737-756.

[7] G. Dal Maso, A. DeSimone, M. G. Mora and M. Morini, A vanishing viscosity approach to quasistatic evolution in plasticity with softening, Arch. Ration. Mech. Anal. 189 (2008), 469-544.

[8] G. Dal Maso, A. DeSimone and F. Solombrino, Quasistatic evolution for cam-clay plasticity: a weak formulation via viscoplastic regularization and time parametrization, Calc. Var. Partial Differential Equations 40 (2011), 125-181.

[9] G. DAl MAso, G. FRANCFORT and R. TOADER, Quasistatic crack growth in nonlinear elasticity, Arch. Ration. Mech. Anal. 176 (2005), 165-225.

[10] M. EFENDIEV and A. MIELKE, On the rate-independent limit of systems with dry friction and small viscosity, J. Convex Anal. 13 (2006), 151-167.

[11] M. EFENDIEV and S. ZELIK, Finite-dimensional attractors and exponential attractors for degenerate doubly nonlinear equations, Math. Methods Appl. Sci. (13) 32 (2009), 16381668.

[12] M. Grasselli, A. Miranville, V. Pata and S. Zelik, Well-posedness and long time behavior of a parabolic-hyperbolic phase-field system with singular potential, Math. Nachr. 280 (2007), 1475-1509.

[13] D. KneEs, A. Mielke and C. ZANini, On the inviscid limit of a model for crack propagation, Math. Models Methods Appl. Sci. 18 (2008), 1529-1569.

[14] D. KNEES, C. ZANini, and A. MielKe, Crack growth in polyconvex materials, Physica D 239 (2010), 1470-1484.

[15] A. MielKe, Evolution in rate-independent systems (Ch. 6), In: "Handbook of Differential Equations, Evolutionary Equations" C. Dafermos and E. Feireisl (eds.), Vol. 2, Elsevier B.V., Amsterdam, 2005, 461-559.

[16] A. MIELKE. Differential, energetic and metric formulations for rate-independent processes, In: "Nonlinear PDE's and Applications", L. Ambrosio and G. Savaré (eds.), Springer, 2011, Vol. 2028, 87-170.

[17] A. MiElKe and F. Theil, On rate-independent hysteresis models, NoDEA Nonlinear Differential Equations Appl. 11 (2004), 151-189.

[18] A. Mielke, A. Petrov and J. A. C. Martins, Convergence of solutions of kinetic variational inequalities in the rate-independent quasi-static limit, J. Math. Anal. Appl. 348 (2008), 1012-1020. 
[19] A. Mielke, R. Rossi and G. Savaré, Modeling solutions with jumps for rateindependent systems on metric spaces, Discr. Cont. Dynam. Systems Ser. A 25 (2009), 585-615.

[20] A. Mielke, R. Rossi and G. Savaré, BV solutions and viscosity approximations of rate-independent systems, ESAIM Control Optim. Calc. Var. 18 (2012), 36-80.

[21] A. MielKe, R. ROSSI and G. SAVARÉ, $B V$ solutions to infinite-dimensional rate-independent systems, WIAS Preprint 1845, 2013, also available at: http://arxiv.org/abs/1309.6291

[22] A. Mielke, F. Theil and V. I. Levitas, A variational formulation of rate-independent phase transformations using an extremum principle, Arch. Ration. Mech. Anal. 162 (2002), 137-177.

[23] A. Miranville and S. ZELIK, Finite-dimensionality of attractors for degenerate equations of elliptic-parabolic type, Nonlinearity 8 (2007), 1773-1797.

[24] S. Nazarov and B. Plamenevsky, "Elliptic Problems in Domains with Piecewise Smooth Boundaries" de Gruyter Expositions in Mathematics, Vol. 13, Walter de Gruyter \& Co., Berlin, 1994.

[25] R. Rossi, A. Mielke and G. SaVAré, A metric approach to a class of doubly nonlinear evolution equations and applications, Ann. Sc. Norm. Super. Pisa Cl. Sci. (5) 7 (2008), 97-169.

[26] G. Schimperna, A. Segatti and U. Stefanelli, Well-posedness and long-time behavior for a class of doubly nonlinear equations, Discr. Cont. Dynam. Systems Ser. A 18 (2008), 15-38.

[27] R. TOADER and C. ZANINI, An artificial viscosity approach to quasistatic crack growth, Boll. Unione Mat. Ital. (9) 2 (2009), 1-36.

[28] H. TRIebel, "Interpolation Theory, Function Spaces, Differential Operators", NorthHolland Publishing Co., Amsterdam, 1978.

[29] E. ZEIDLER, "Nonlinear Functional Analysis and its Applications. II/B", Nonlinear Monotone Operators, translated from the German by the author and Leo F. Boron, SpringerVerlag, New York, 1990.

Weierstraß-Institut

für Angewandte Analysis und Stochastik

Mohrenstraße 39

D-10117 Berlin, Germany

and

Institut für Mathematik

Humboldt-Universität zu Berlin

Rudower Chaussee 25

D-12489 Berlin, Germany

alexander.mielke@wias-berlin.de

University of Surrey

Department of Mathematics

Guildford

GU2 7XH, United Kingdom

s.zelik@surrey.ac.uk 